\title{
THE SMASH PRODUCT OF MONOIDAL THEORIES
}

\author{
Amar Hadzihasanovic
}

Tallinn University of Technology

\begin{abstract}
The tensor product of props was defined by Hackney and Robertson as an extension of the BoardmanVogt product of operads to more general monoidal theories. Theories that factor as tensor products include the theory of commutative monoids and the theory of bialgebras. We give a topological interpretation (and vast generalisation) of this construction as a low-dimensional projection of a "smash product of pointed directed spaces". Here directed spaces are embodied by combinatorial structures called diagrammatic sets, while Gray products replace cartesian products. The correspondence is mediated by a web of adjunctions relating diagrammatic sets, pros, probs, props, and Gray-categories. The smash product applies to presentations of higher-dimensional theories and systematically produces higher-dimensional coherence cells.
\end{abstract}

Current version: 27th January 2021

\section{CONTEnTS}

1. Some higher structures........ 9

2. Monoidal theories............. 19

3. Combinatorial results........... 28

4. Pros and diagrammatic sets ... 36

5. The smash product ............ $4^{8}$

6. Higher-dimensional cells ...... 65

\section{INTRODUCTION}

In a categorical tradition of universal algebra dating back to F. William Lawvere's thesis [Law63], algebraic theories are embodied by cartesian monoidal categories whose objects are freely generated from a set of sorts. Models 
of the theory, embodied by strong monoidal functors, may live in an arbitrary cartesian monoidal category: we specialise to the category of sets and functions to recover the classical notion of model.

Following this fundamental shift in perspective, and considering that cartesianness of a monoidal structure can be defined equationally [Fox76], it is a relatively small step to consider more general monoidal theories whose models live in arbitrary monoidal categories.

Monoidal theories are embodied by structures called pros [ML65]. ${ }^{1}$ Intermediate between monoidal theories and algebraic theories, there are braided and symmetric monoidal theories, embodied respectively by probs and props. The familiar term-algebraic calculus is inadequate for these generalised theories, and is commonly replaced by a calculus of string diagrams [Sel10].

It is common for a mathematical object to have both a structure of $T$-model and of $S$-model for some theories $T, S$, satisfying some compatibility condition. For example, a bimodule is both a left and a right module, in such a way that the left and right actions commute. A natural question is: can we systematically compose theories, so that a model of the composite of $T$ and $S$ is an object with compatible $T$ and $S$-model structures?

In a line of work that has been attracting attention in theoretical computer science [BSZ14], composition of monoidal theories is mediated by distributive laws which specify a factorisation system between operations of $T$ and $S$, as described by Steve Lack [Lac04].

A less flexible, yet more uniform composition is the tensor product of props, which applies to all props and does not require additional data. The tensor product was defined and studied by Philip Hackney and Marcy Robertson $\left[\right.$ HR15], ${ }^{2}$ who also proved that it extends, in a precise sense, the product of symmetric operads introduced by J. M. Boardman and R. M. Vogt [BV06].

Some intuition about the tensor product may be gained as follows. If $\mathbf{M}$ is a symmetric monoidal category, the category of $T$-models in $\mathbf{M}$ inherits a symmetric monoidal structure: to compose two models, we "run their operations in parallel", using the symmetric structure of $\mathbf{M}$ to rearrange inputs and outputs as needed. The data of a model in $\mathbf{M}$ of the tensor product $T \otimes_{\mathbb{S}} S$ is equivalent to the data of an $S$-model in the category of $T$-models in $\mathbf{M}^{3}{ }^{3}$

As remarked in [Lac04, §4.2], there is something mysterious about the rôle of symmetric braidings in the composition of monoidal theories. From a certain

\footnotetext{
${ }^{1}$ In some sources, the term pro or PRO is reserved for a one-sorted theory, and the multi-sorted variant is called a coloured pro.

${ }^{2}$ Although its possibility was noticed earlier by John C. Baez [Bae06]. Baez's lectures are also a nice survey of the relations between pros, probs, props, and algebraic theories.

${ }^{3} \mathrm{Or}$, symmetrically, a $T$-model in the category of $S$-models in $\mathrm{M}$.
} 
perspective, a symmetric braiding is just another operation in a pro, yet it plays an inescapable structural rôle in the tensor product.

Consider the theories of monoids and comonoids. These are planar monoidal theories, naturally embodied by a pro: in the corresponding prop, symmetric braidings are added freely, so models in the sense of props are equivalent to models in the sense of pros. Nevertheless, their tensor product - the theory of bialgebras - features the non-planar equation

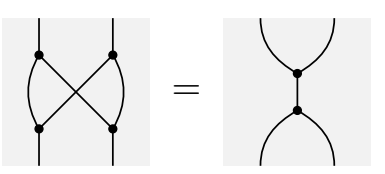

where the symmetric braiding in the left-hand side cannot clearly be attributed to either factor.

In particular, the tensor product of props does not restrict to a monoidal structure on pros. At most, as shown in Section 5.1, we can define an "external" tensor product which takes two pros and returns a prob, from which we can then universally reconstruct the tensor product of props.

A few years ago, we noticed that equation (1) admits the following topological interpretation. ${ }^{4}$ Take the string diagrams corresponding to monoid multiplication and comonoid comultiplication, and extend them along perpendicular directions in the plane so that they form branching surfaces:
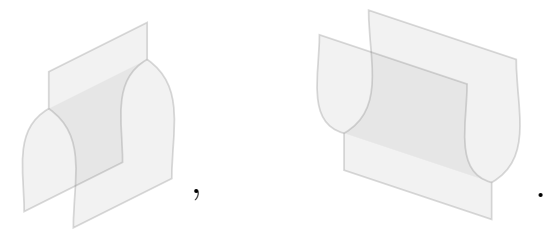

Intersect the two branching surfaces and "slide" one past another along the vertical axis. As one branching slides past the other branching, the intersection - a "string diagram in 3-dimensional space" - evolves as in the following figure:

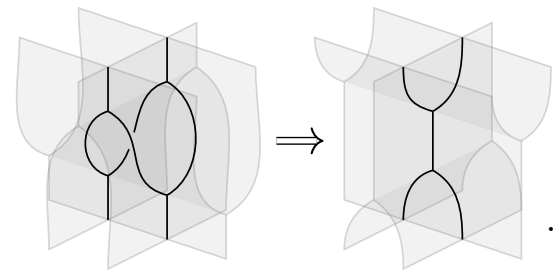

The two sides of (1) arise as planar projections of the two sides of (2).

\footnotetext{
${ }^{4} \mathrm{~A}$ similar observation was made, around the same time, by J. Scott Carter [Car18].
} 
This interpretation extends to all "compatibility" equations in the tensor product of props, and recasts the tensor product as a dimension-raising construction: given planar diagrams, it produces equations of 3-dimensional diagrams. This solves our conundrum about braidings: they are absent in the 3 -dimensional picture, and only appear in the 2-dimensional picture as an artefact of planar projection.

What is going on? As first suggested in [Had17, Section 2.3], the correct interpretation of (2) is that it arises from a smash product of pointed directed spaces, in a sense that we will soon explain.

Our model of directed space is a diagrammatic set [Had20b]. We developed the theory of diagrammatic sets partly as a foundation for this work, which requires the ability to do rewriting and diagrammatic reasoning in weak higher categories of arbitrary dimension, to an extent that pre-existing frameworks did not seem to support.

The aim of this article is the statement and proof of Theorem 5.29: the "external" tensor product of pros factors functorially through the smash product of pointed diagrammatic sets. Through this result, we can attribute a precise meaning to our earlier statements, such as the assertion that equation (1) arises from (2). On our way, we develop a great deal of combinatorics in order to relate diagrammatic sets, pros, and probs through a web of adjunctions involving a few "ancillary" higher structures.

We see this result not as an end point, but as an opening. Far from being just a reinterpretation, our smash product is a vast generalisation of the tensor product of props, and transitively of the Boardman-Vogt product of operads.

Indeed, pointed diagrammatic sets can embody higher-dimensional theories with non-invertible generators in arbitrarily high dimension. ${ }^{5}$ From these, the smash product generates non-invertible higher-dimensional cells rather than equations.

Already when applied to 3-dimensional presentations of monoidal theories [Mim14], not only this construction produces a presentation of their tensor product, that is, it produces oriented equations, or rewrites; it also produces interesting higher-dimensional coherence cells, or syzygies, up to dimension 6 .

In higher-dimensional rewriting and universal algebra [GM16, Gui19], coherence is usually pursued with analytic methods of rewriting theory such as the computation of critical branchings. We believe that our results may be a gateway to new synthetic and compositional methods.

\footnotetext{
${ }^{5}$ As opposed to structures used in homotopical algebra, such as $\infty$-operads, that embody theories with invertible higher data.
} 
Monoidal theories, directed spaces, and diagrammatic sets

The connection between monoidal theories and directed spaces is based on four conceptual leaps. The first leap, as mentioned, is the realisation that monoidal categories can embody algebraic theories.

The second leap is John C. Baez and James Dolan's formulation of the periodic table of $n$-categories, by which a monoidal category is equivalent to a bicategory with a single 0-cell, but a braided monoidal category is equivalent to a tricategory with a single 0-cell and 1-cell [BD95]. A variant of this result implies that a pro is a special kind of 2-category, while a prob is a special kind of Gray-category, a semistrict notion of tricategory [GPS95]. This matches the intuition that tensoring pros to obtain a prob is dimension-raising.

The third leap is Grothendieck's homotopy hypothesis, that "spaces", more precisely homotopy types, are modelled in a precise combinatorial sense by higher groupoids. In models where higher groupoids are higher categories whose cells are all invertible in a weak sense, this leaves open the possibility of interpreting higher categories as "spaces of directed cells".

The fourth and final leap is Albert Burroni's observation that various notions of presentations by generators and relations, or rewrite systems, can be unified as presentations of "cell complexes in a category of higher categories", a notion of directed space with combinatorial structure [Bur93].

Following the sequence, we can reinterpret a monoidal theory with its set of sorts as a kind of directed 2-dimensional space containing a 1-dimensional cell complex. A braided monoidal theory is the same thing one dimension up.

These spaces are canonically pointed with the unique 0 -cell in the cell complex structure. It is natural, at this point, to wonder about a directed counterpart of the classical smash product of pointed spaces. The correct generalisation replaces the cartesian product of spaces with a version of the Gray product [Gra74].

In $[\operatorname{Had} 17$, Section 2.3], we considered smash products in the context of Burroni and Ross Street's theory of polygraphs, based on strict $\omega$-categories. This had the advantage that a theory of Gray products had already been developed [Ste04, AM20], and that we could identify a pro directly with a pointed 2-category. However, in this context the smash product of pros produces a strict 3-category equivalent not to a braided monoidal category, but to a highly degenerate commutative monoidal category. ${ }^{6}$

In [Had20b], based on an abandoned idea of Mikhail Kapranov and Vladimir Voevodsky, we developed the theory of diagrammatic sets as an alternative to

\footnotetext{
${ }^{6}$ This is connected to the known failure of the homotopy hypothesis for strict $\omega$-categories, see [Sim09, Chapter 4].
} 
polygraphs that would avoid this pitfall and support rewriting and diagrammatic reasoning in weak higher categories.

While the model of a directed cell in a polygraph is algebraic, diagrammatic sets adopt a combinatorial model. Roughly, a model of a directed $n$-cell is the face poset of a regular CW-decomposition of the topological closed $n$-ball, together with an orientation subdividing the boundary of each cell into an input and output half, in such a way that the input and output half are also face posets of regular CW-balls, and their orientations determine a composable pasting diagram in a strict $\omega$-category. Common higher-categorical shapes such as oriented simplices and cubes appear as special cases.

A pleasant outcome of the transition to the combinatorial setup is that Gray products and smash products are much easier to define and compute. On the other hand, the identification of pros or probs with certain pointed diagrammatic sets is non-trivial. The technical core of this article is the definition of a full and faithful diagrammatic nerve of pros (Section 4.1), and then of a non-trivial ${ }^{7}$ realisation functor of diagrammatic sets in Gray-categories (Section 4.2), which allows us to recover the tensor product of two pros as the realisation of the smash product of their nerves. ${ }^{8}$

Diagrammatic sets are related to (nice) topological spaces by a nerve and realisation pair [Had20b, Section 8.3], where the nerve realises a version of the homotopy hypothesis. As detailed in Section 5.2, the geometric realisation sends Gray products to cartesian products, so it sends smash products to smash products.

Altogether, our results amount to the surprising fact that the tensor product of pros and the smash product of pointed spaces are two facets of the same construction.

\section{Related work}

We have paid tribute to our main influences on the conceptual side. On the other hand, this article is technically most indebted to three sources.

The first is Hackney and Robertson's article on the category of props [HR15]: beyond the fact that they defined the tensor product of props, our proofs in Section 2 that certain categories of pros and probs have small limits and colimits are essentially lifted from their work on props, with minor tweaks.

The second is John Power's work on pasting diagrams [Pow91]. While our formalisation of diagrams is based on Richard Steiner's combinatorial frame-

\footnotetext{
${ }^{7}$ There is also a "trivial" functor passing through strict 3-categories.

${ }^{8}$ More precisely, of their nerves with an orientation reversal in the second factor, as explained in Section 5.3.
} 
work [Ste93], an analogue of Power's domain replacement condition turns out to be key to the constructions of Section 4, and the technical Section 3 is devoted to showing that it holds for all our 3-dimensional diagrams.

In particular, our Theorem 3.24 is roughly equivalent in meaning to [Pow91, Theorem 4.14]. Interestingly, though, Power's topological setup seems to have completely different strengths and weaknesses compared to our combinatorial setup: when translating Power's proofs, we discovered that every single non-trivial step in his proofs followed easily from our definitions, whereas the trivial steps would require non-trivial proofs, as in Proposition 3.21. No formal comparison has been made, to our knowledge, between Steiner's and Power's theory, so we think it is justified to consider our results original.

The third is Simon Forest and Samuel Mimram's article on the rewriting theory of Gray-categories [FM18]. Not only we learnt from them a convenient axiomatisation of Gray-categories, but the construction of Section 4.2 draws directly on their ideas and results and can be seen as a continuation of their work, showing that every diagrammatic set presents a Gray-category.

\section{Structure of the article}

Most of the article is aimed at the proof of Theorem 5.29.

The statement of this result involves many different structures, related via a number of ancillary structures, each in need of definition. Some of these are obscure enough that basic technical aspects could not be found in the literature and had to be developed expressly. That said, we tried to keep redundancy to a minimum by treating a structure as a special case of another whenever possible, even if it results in unconventional choices, such as the definition of reflexive $\omega$-graphs after diagrammatic sets.

Section 1 recaps the elementary theory of directed complexes, diagrammatic sets, and strict $\omega$-categories. Section 2 introduces categories of pros, probs, and props, proves some of their properties, and clarifies the relation between probs and Gray-categories. Section 3 proves some technical results about directed complexes in low dimension. Section 4 is the technical core of the article, constructing the adjunctions that relate diagrammatic sets, pros, and Gray-categories. Section 5 defines the tensor product of props and the smash product of pointed diagrammatic sets, then proves the main theorem. Section 6 takes the first steps into diagrammatic sets as a framework for higher-dimensional rewriting and universal algebra.

Every reader should get at least acquainted with the definitions in the first two sections. On a first read, they can then skip to Section 5, using the diagram that concludes Section 4 as a reference: most of the time, knowing 
that certain functors exist and are left or right adjoints should be enough to follow the outline of the proof.

Some readers may be content with understanding the picture (2) and want to stop there. Those interested in higher-dimensional rewriting and universal algebra should move on to Section 6 .

Section 3 may appeal to the reader who appreciates the combinatorics of higher-categorical diagrams. The reader who enjoyed Forest and Mimram's [FM18] can read Section 4 as a follow-up of sorts.

We use the diagrammatic order $f ; g$ for the composition of morphisms $f$ and $g$ in a category, but the "classical" order GF for the composition of functors $\mathrm{F}$ and G. Other notational choices are explained when they are introduced.

Outlook and open problems

Section 6 is an extended outlook towards our main prospect, namely, the introduction of new compositional methods in higher-dimensional rewriting and universal algebra.

We briefly mention other potential developments. Christoph Dorn, David Reutter, and Jamie Vicary have defined a semistrict algebraic model of $n$-categories, called associative n-categories, which is equivalent to Gray-categories for $n=3$ [Dor18, RV19]. It is conceivable that the adjunction of Section 4.2 relating diagrammatic sets to Gray-categories may generalise to associative $n$-categories for $n>3$. We note, however, that our construction uses a property, frame acyclicity, which holds in general up to dimension 3 but fails in dimension 4 or higher, so it is likely that new ideas will be needed.

The theory of diagrammatic sets is based on simple data structures: a cell model $U$ can be encoded as the directed graph $\mathscr{H}^{\circ} U$ of $\S 3.12$ together with a grading of its vertices; the Gray product is then encoded as a cartesian product of directed graphs, ${ }^{9}$ while the degrees of vertices are summed. We expect that this setup should lend itself to computational formalisation.

This is of particular interest considering that the theory of associative $n$-categories is formalised in the graphical proof assistant homotopy.io: implementing the constructions of Section 4.2 would give us access to visualisations of Gray and smash products through this graphical frontend.

\footnotetext{
${ }^{9}$ With some edges reversed, depending on the degree of first factor.
} 


\section{Some Higher STRUCTURES}

\subsection{Directed complexes and diagrammatic sets}

We quickly go through the main definitions, and refer the reader to [Had20b] for an in-depth development.

1.1 (Graded poset). Let $P$ be a finite poset with order relation $\leq$. For all elements $x, y \in P$, we say that $y$ covers $x$ if $x<y$ and, for all $y^{\prime} \in X$, if $x<y^{\prime} \leq y$ then $y^{\prime}=y$.

The Hasse diagram of $P$ is the finite directed graph $\mathscr{H} P$ with $\mathscr{H} P_{0}:=P$ as set of vertices and $\mathscr{H} P_{1}:=\{y \rightarrow x \mid y$ covers $x\}$ as set of edges.

Let $P_{\perp}$ be $P$ extended with a least element $\perp$. We say that $P$ is graded if, for all $x \in P$, all directed paths from $x$ to $\perp$ in $\mathscr{H} P_{\perp}$ have the same length. If this length is $n+1$, we let $\operatorname{dim}(x):=n$ be the dimension of $x$.

1.2 (Closed and pure subsets). Let $P$ be a poset and $U \subseteq P$. The closure of $U$ is the subset $\operatorname{cl} U:=\{x \in P \mid \exists y \in U x \leq y\}$ of $P$. We say that $U$ is closed if $U=\operatorname{cl} U$.

Suppose $P$ is graded and $U \subseteq P$ is closed. Then $U$ is graded with the partial order inherited from $P$. The dimension $\operatorname{dim}(U)$ of $U$ is $\max \{\operatorname{dim}(x) \mid x \in U\}$ if $U$ is inhabited, -1 otherwise. In particular, $\operatorname{dim}(\operatorname{cl}\{x\})=\operatorname{dim}(x)$.

We say that $U$ is pure if its maximal elements all have dimension $\operatorname{dim}(U)$.

1.3 (Oriented graded poset). An orientation on a finite poset $P$ is an edgelabelling $o: \mathscr{H} P_{1} \rightarrow\{+,-\}$ of its Hasse diagram.

An oriented graded poset is a finite graded poset with an orientation.

1.4. We will often let variables $\alpha, \beta$ range implicitly over $\{+,-\}$.

1.5 (Boundaries). Let $P$ be an oriented graded poset and $U \subseteq P$ a closed subset. Then $U$ inherits an orientation from $P$ by restriction.

For all $\alpha \in\{+,-\}$ and $n \in \mathbb{N}$, we define

$$
\begin{aligned}
\Delta_{n}^{\alpha} U & :=\{x \in U \mid \operatorname{dim}(x)=n \text { and if } y \in U \text { covers } x, \text { then } o(y \rightarrow x)=\alpha\}, \\
\partial_{n}^{\alpha} U & :=\operatorname{cl}\left(\Delta_{n}^{\alpha} U\right) \cup\{x \in U \mid \text { for all } y \in U, \text { if } x \leq y, \text { then } \operatorname{dim}(y) \leq n\}, \\
\Delta_{n} U & :=\Delta_{n}^{+} U \cup \Delta_{n}^{-} U, \quad \partial_{n} U:=\partial_{n}^{+} U \cup \partial_{n}^{-} U .
\end{aligned}
$$

We call $\partial_{n}^{-} U$ the input $n$-boundary and $\partial_{n}^{+} U$ the output n-boundary of $U$.

If $U$ is $(n+1)$-dimensional, we write $\Delta^{\alpha} U:=\Delta_{n}^{\alpha} U$ and $\partial^{\alpha} U:=\partial_{n}^{\alpha} U$. For each $x \in P$, we write $\Delta_{n}^{\alpha} x:=\Delta_{n}^{\alpha} \operatorname{cl}\{x\}$ and $\partial_{n}^{\alpha} x:=\partial_{n}^{\alpha} \operatorname{cl}\{x\}$. 
1.6 (Atoms and molecules). Let $P$ be an oriented graded poset. We define a family of closed subsets of $P$, the molecules of $P$, by induction on proper subsets. If $U$ is a closed subset of $P$, then $U$ is a molecule if either

- $U$ has a greatest element, in which case we call it an atom, or

- there exist molecules $U_{1}$ and $U_{2}$, both properly contained in $U$, and $n \in \mathbb{N}$ such that $U_{1} \cap U_{2}=\partial_{n}^{+} U_{1}=\partial_{n}^{-} U_{2}$ and $U=U_{1} \cup U_{2}$.

We define $\sqsubseteq$ to be the smallest partial order relation such that, if $U_{1}$ and $U_{2}$ are molecules and $U_{1} \cap U_{2}=\partial_{n}^{+} U_{1}=\partial_{n}^{-} U_{2}$, then $U_{1}, U_{2} \sqsubseteq U_{1} \cup U_{2}$.

We say $n$-molecule for an $n$-dimensional molecule. We say that $P$ itself is a molecule if $P \subseteq P$ is a molecule.

1.7 (Spherical boundary). An $n$-molecule $U$ in an oriented graded poset has spherical boundary if, for all $k<n$,

$$
\partial_{k}^{+} U \cap \partial_{k}^{-} U=\partial_{k-1} U
$$

1.8 (Regular directed complex). An oriented graded poset $P$ is a regular directed complex if, for all $x \in P$ and $\alpha, \beta \in\{+,-\}$,

1. $\operatorname{cl}\{x\}$ has spherical boundary,

2. $\partial^{\alpha} x$ is a molecule, and

3. $\partial^{\alpha}\left(\partial^{\beta} x\right)=\partial_{n-2}^{\alpha} x$ if $n:=\operatorname{dim}(x)>1$.

A map $f: P \rightarrow Q$ of regular directed complexes is a function of their underlying sets that satisfies

$$
\partial_{n}^{\alpha} f(x)=f\left(\partial_{n}^{\alpha} x\right)
$$

for all $x \in P, n \in \mathbb{N}$, and $\alpha \in\{+,-\}$. We call an injective map an inclusion. With their maps, regular directed complexes form a category $\mathbf{D C} \mathbf{p} \mathbf{x}^{\mathcal{R}}$.

Remark 1.9. As shown in [Had20b, Section 1.3], $\mathbf{D C} \mathbf{p x x}^{\mathcal{R}}$ has an initial object, a terminal object, and pushouts of inclusions.

1.10 (Regular molecule). A regular molecule is a molecule which is a regular directed complex.

By [Had20b, Proposition 1.38], if two regular molecules are isomorphic in $\mathbf{D C} \mathbf{p} \mathbf{x}^{\mathcal{R}}$, they are isomorphic in a unique way. As customary in these situations, we will treat isomorphic regular molecules as "equal" under appropriate circumstances.

1.11 (Globe). For each $n \in \mathbb{N}$, let $O^{n}$ be the poset with a pair of elements $\underline{k}^{+}, \underline{k}^{-}$for each $k<n$ and a greatest element $\underline{n}$, with the partial order defined 
by $\underline{j}^{\alpha} \leq \underline{k}^{\beta}$ if and only if $j \leq k$. This is a graded poset, with $\operatorname{dim}(\underline{n})=n$ and $\operatorname{dim}\left(\underline{k}^{\alpha}\right)=k$ for all $k<n$.

With the orientation $o\left(y \rightarrow \underline{k}^{\alpha}\right):=\alpha$ if $y$ covers $\underline{k}^{\alpha}, O^{n}$ becomes a regular directed complex, in particular a regular atom. We call $O^{n}$ the $n$-globe.

1.12 (Pasting of molecules). Let $U_{1}, U_{2}$ be regular molecules and suppose that $\partial_{k}^{+} U_{1}$ and $\partial_{k}^{-} U_{2}$ are isomorphic in $\mathbf{D} \mathbf{C} \mathbf{p} \mathbf{p x}^{\mathcal{R}}$. Given an isomorphic copy $V$ of the two, there is a unique span of inclusions $V \hookrightarrow U_{1}$ and $V \hookrightarrow U_{2}$ whose images are, respectively, $\partial_{k}^{+} U_{1}$ and $\partial_{k}^{-} U_{2}$. We let $U_{1} \#_{k} U_{2}$ be the pushout

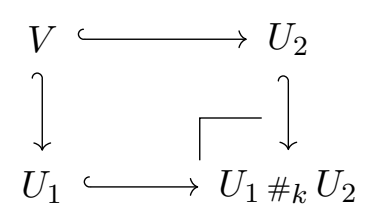

in $\mathbf{D C} \mathbf{p} \mathbf{x}^{\mathcal{R}}$. Then $U_{1} \#_{k} U_{2}$ is a regular molecule, decomposing as $U_{1} \cup U_{2}$ with $U_{1} \cap U_{2}=\partial_{k}^{+} U_{1}=\partial_{k}^{-} U_{2}$.

1.13 ( $-\Rightarrow-$ construction). Let $U, V$ be regular $n$-molecules with spherical boundary such that $\partial^{\alpha} U$ is isomorphic to $\partial^{\alpha} V$ for all $\alpha \in\{+,-\}$.

Form the pushout $U \cup V$ of the span of inclusions $\partial U \hookrightarrow U, \partial U \hookrightarrow V$ whose images are $\partial U$ and $\partial V$, respectively. We define $U \Rightarrow V$ to be the oriented graded poset obtained from $U \cup V$ by adjoining a greatest element $\top$ with $\partial^{-} \top:=U$ and $\partial^{+} \top:=V$. Then $U \Rightarrow V$ is an $(n+1)$-dimensional atom with spherical boundary.

$1.14(\langle-\rangle$ construction). Let $U$ be a regular molecule with spherical boundary. Then $\partial^{-} U \Rightarrow \partial^{+} U$ is defined, and we denote it by $\langle U\rangle$.

1.15. There is a unique 0 -atom, namely, the 0 -globe $1:=O^{0}$, which is also the terminal object of $\mathbf{D C p} \mathbf{x}^{\mathcal{R}}$.

We define a sequence $\left\{I_{n}\right\}_{n>0}$ of 1-molecules by

$$
I_{1}:=O^{1}, \quad I_{n}:=I_{n-1} \#_{0} O^{1} \text { for } n>1 .
$$

Every regular 1-molecule is of the form $I_{n}$ for some $n>0$.

For each pair $n, m>0$, let $U_{n, m}:=\left(I_{n} \Rightarrow I_{m}\right)$. Every regular 2-atom is of the form $U_{n, m}$ for some $n, m>0$. Regular 2-molecules are then generated by $I_{1}$ and the $U_{n, m}$ under the pasting operations $\#_{0}, \#_{1}$.

1.16 (Diagrammatic set). We write $\odot$ for a skeleton of the full subcategory of $\mathbf{D C p x}{ }^{\mathcal{R}}$ on the atoms of every dimension.

A diagrammatic set is a presheaf on $\odot$. Diagrammatic sets and their morphisms of presheaves form a category $\odot$ Set. 
Comment 1.17. The definition in $[\operatorname{Had} 20 \mathrm{~b}]$ is relative to a fixed "convenient" class of molecules; for simplicity, here we pick the class of all molecules with spherical boundary.

1.18. We identify $\odot$ with a full subcategory $\odot \hookrightarrow \odot$ Set via the Yoneda embedding. With this identification, we use morphisms in $\odot$ Set as our notation for both elements and structural operations of a diagrammatic set $X$ :

- $x \in X(U)$ becomes $x: U \rightarrow X$, and

- for each map $f: V \rightarrow U$ in $\odot, X(f)(x) \in X(V)$ becomes $f ; x: V \rightarrow X$.

As described in [Had20b, §4.4], the embedding $\odot \hookrightarrow \odot$ Set extends to an embedding $\mathbf{D C} \mathbf{p x x}^{\mathcal{R}} \hookrightarrow \odot$ Set.

1.19 (Diagrams and cells). Let $X$ be a diagrammatic set and $U$ a regular molecule. A diagram of shape $U$ in $X$ is a morphism $x: U \rightarrow X$. It is composable if $U$ has spherical boundary and a cell if $U$ is an atom. For all $n \in \mathbb{N}$, we say that $x$ is $n$-diagram or an $n$-cell when $\operatorname{dim}(U)=n$.

If $U$ decomposes as $U_{1} \#_{k} U_{2}$, we write $x=x_{1} \#_{k} x_{2}$ for $x_{i}:=\imath_{i} ; x$, where $\imath_{i}$ is the inclusion $U_{i} \hookrightarrow U$ for $i \in\{1,2\}$. This extends associatively to $n$-ary decompositions for $n>2$.

If $x: U \rightarrow X$ is a diagram in $X$ and $f: X \rightarrow Y$ a morphism of diagrammatic sets, we may write $f(x)$ for the diagram $x ; f: U \rightarrow Y$.

1.20 (Boundaries of diagrams). Let $X$ be a diagrammatic set, $x: U \rightarrow X$ a diagram, and let $\imath_{k}^{\alpha}: \partial_{k}^{\alpha} U \hookrightarrow U$ be the inclusions of the $k$-boundaries of $U$. The input $k$-boundary of $x$ is the diagram $\partial_{k}^{-} x:=\imath_{k}^{-} ; x$ and the output $k$-boundary of $x$ is the diagram $\partial_{k}^{+} x:=\imath_{k}^{+} ; x$. We may omit the index $k$ when $k=\operatorname{dim}(U)-1$.

We write $x: y^{-} \Rightarrow y^{+}$to express that $\partial_{k}^{\alpha} x=y^{\alpha}$ for each $\alpha \in\{+,-\}$, and say that $x$ is of type $y^{-} \Rightarrow y^{+}$. We say that two diagrams $x_{1}, x_{2}$ are parallel if they have the same type.

1.21. A 1-cell $a$ in a diagrammatic set has shape $I_{1}$. A 2-cell $\varphi$ has shape $U_{n, m}$ for some $n, m>0$, so it is of type

$$
a_{1} \#_{0} \ldots \#_{0} a_{n} \Rightarrow b_{1} \#_{0} \ldots \#_{0} b_{m}
$$

for some 1-cells $a_{1}, \ldots, a_{n}, b_{1}, \ldots, b_{m}$. We may depict such cells as string diagrams
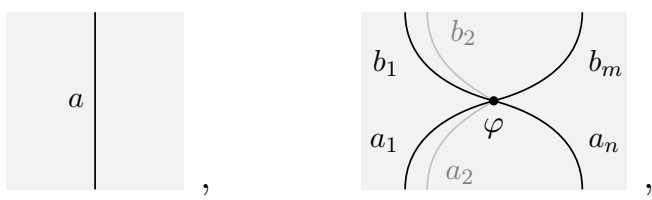
where a lighter shade indicates a repeated pattern. Each region bounded by wires corresponds to a potentially different 0 -cell; in practice, we will mostly work with diagrammatic sets that have a single 0-cell. Labels will be omitted when irrelevant, or implied by the shape of a cell.

A 2-diagram decomposes into 1-cells and 2-cells under the $\#_{0}, \#_{1}$ operations. In string diagrams, $\#_{0}$ is horizontal juxtaposition and $\#_{1}$ is vertical juxtaposition with the output wires of one diagram connecting to the input wires of another. For example,

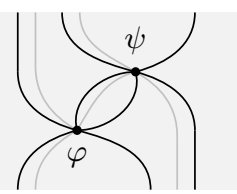

depicts a generic 2-diagram of the form

$$
\left(\varphi \#_{0} a_{1} \#_{0} \ldots \#_{0} a_{n}\right) \#_{1}\left(b_{1} \#_{0} \ldots \#_{0} b_{m} \#_{0} \psi\right)
$$

for some 2-cells $\varphi, \psi$ and 1 -cells $a_{1}, \ldots, a_{n}, b_{1}, \ldots, b_{m}$.

Observe that, in our setting, there is no need to attribute a topological nature to string diagrams, à la Joyal and Street [JS91]: they should instead be interpreted as compact encodings of regular molecules - a discrete, combinatorial structure - and their morphisms to diagrammatic sets.

A 2-diagram is composable if and only if it is connected as a string diagram. For example, of the diagrams
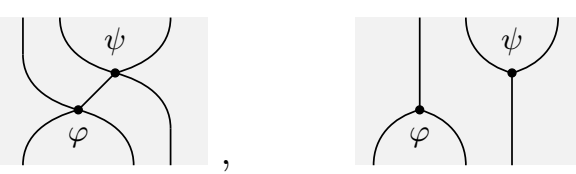

only the first one is composable.

We may depict a 3-diagram as a sequence of rewrites on composable subdiagrams of a diagram. For example, a diagrammatic set with a single 0-cell, a single 1-cell, and 3-cells $\varphi, \psi$ of the form
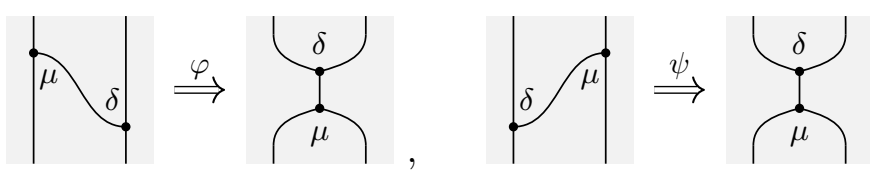

admits a 3-diagram of the form

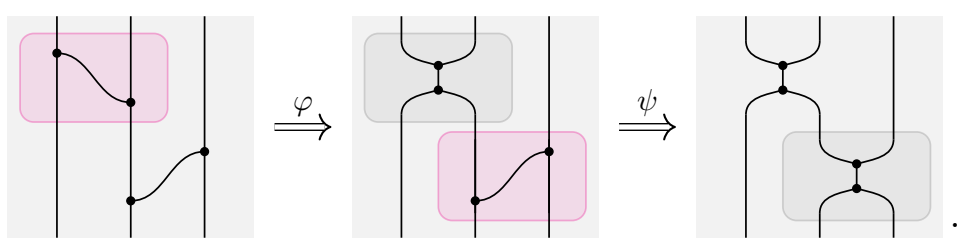


where the input boundary of each 3-cell is highlighted in pink.

We will also use string diagrams to describe certain regular molecules directly. This is justified by the interpretation of a molecule $U$ as the "tautologous" diagram $\operatorname{id}_{U}: U \rightarrow U$ in $\odot$ Set.

1.22 (Dual diagrammatic set). Let $U$ be a regular atom. The oriented graded poset $U^{\circ}$ with the same underlying poset as $U$ and the opposite orientation $o^{\circ}(y \rightarrow x):=-o(y \rightarrow x)$ is a regular atom. If $f: U \rightarrow V$ is a map in $\odot$, its underlying function also defines a map $f^{\circ}: U^{\circ} \rightarrow V^{\circ}$. This determines an involution $-{ }^{\circ}$ on $\odot$.

Let $X$ be a diagrammatic set. Its dual $X^{\circ}$ is the diagrammatic set defined by $X^{\circ}(-):=X\left(-^{\circ}\right)$. This extends to morphisms in the obvious way, and extends the involution on $\odot$ to an involution on $\odot$ Set.

Remark 1.23. If $x: U \rightarrow X$ is a 2-diagram, the depiction of $x^{\circ}: U^{\circ} \rightarrow X^{\circ}$ in string diagrams is the horizontal and vertical reflection of the depiction of $x$.

\subsection{Higher-categorical structures}

1.24 (Reflexive $\omega$-graph). Let $\mathbf{O}$ be the full subcategory of $\odot$ whose objects are the globes $O^{n}$. For all $n$ and $k<n$,

- the $k$-boundary inclusions $\imath_{k}^{+}, \imath_{k}^{-}$are the only inclusions of $O^{k}$ into $O^{n}$;

- the map $\tau: O^{n} \rightarrow O^{k}$, defined by $\tau(\underline{n}), \tau\left(\underline{j}^{\alpha}\right):=\underline{k}$ if $j \geq k$ and $\tau\left(\underline{j}^{\alpha}\right):=\underline{j}^{\alpha}$ if $j<k$, is the only surjective map from $O^{n}$ onto $O^{k}$.

A reflexive $\omega$-graph is a presheaf $X$ on $\mathbf{O}$. With their morphisms of presheaves, reflexive $\omega$-graphs form a category $\omega \mathbf{G} \mathbf{p h} \mathbf{h}_{r e f}$.

1.25. The embedding $\mathbf{O} \hookrightarrow \odot$ induces a restriction functor $\odot$ Set $\rightarrow \omega \mathbf{G p h}_{\text {ref }}$ with a full and faithful left adjoint $\omega \mathbf{G p h}_{\text {ref }} \hookrightarrow \odot$ Set; we can thus identify reflexive $\omega$-graphs with particular diagrammatic sets, and use for them the same terminology and notation.

Because all $n$-cells in a reflexive $\omega$-graph $X$ have the same shape $O^{n}$, we leave it implicit and write $X_{n}:=X\left(O^{n}\right)$.

1.26 (Units). Let $x$ be a $k$-cell in a reflexive $\omega$-graph $X$. For $n>k$, we let $\varepsilon_{n} x:=\tau ; x$ where $\tau$ is the unique surjective map $O^{n} \rightarrow O^{k}$. We call $\varepsilon_{n} x$ a unit on $x$. We may omit the index when $n=k+1$.

1.27 (Rank of a cell). Let $x$ be an $n$-cell in a reflexive $\omega$-graph. The rank $\operatorname{rk}(x)$ of $x$ is defined inductively on $n$ as follows:

- if $n=0$, then $\operatorname{rk}(x):=0$; 
- if $n>0$, if $x=\varepsilon y$ for an $(n-1)$-cell $y$, then $\operatorname{rk}(x):=\operatorname{rk}(y)$, otherwise $\operatorname{rk}(x):=n$.

1.28 (Partial $\omega$-category). A partial $\omega$-category is a reflexive $\omega$-graph $X$ together with partial $k$-composition operations

$$
\#_{k}: X_{n} \times X_{n} \rightarrow X_{n}
$$

for all $n \in \mathbb{N}$ and $k<n$, satisfying the following axioms:

1. for all $n$-cells $x, y$ and all $k<n$ such that $x \#_{k} y$ is defined,

$$
\partial_{k}^{+} x=\partial_{k}^{-} y \text { and } \varepsilon\left(x \#_{k} y\right)=\varepsilon x \#_{k} \varepsilon y \text {; }
$$

2. for all $n$-cells $x$ and all $k<n$, the $k$-compositions

$$
x \#_{k} \varepsilon_{n}\left(\partial_{k}^{+} x\right) \text { and } \varepsilon_{n}\left(\partial_{k}^{-} x\right) \#_{k} x
$$

are defined and equal to $x$;

3. for all $(n+1)$-cells $x, y$ and $k<n$, whenever the left-hand side is defined, the right-hand side is defined and

$$
\begin{aligned}
\partial^{-}\left(x \#_{n} y\right) & =\partial^{-} x, \\
\partial^{+}\left(x \#_{n} y\right) & =\partial^{+} y, \\
\partial^{\alpha}\left(x \#_{k} y\right) & =\partial^{\alpha} x \#_{k} \partial^{\alpha} y
\end{aligned}
$$

4. for all cells $x, y, z$ and all $k$ such that both sides are defined,

$$
\left(x \#_{k} y\right) \#_{k} z=x \#_{k}\left(y \#_{k} z\right)
$$

5. for all cells $x, y, x^{\prime}, y^{\prime}$, all $n$ and all $k<n$ such that both sides are defined,

$$
\left(x \#_{n} x^{\prime}\right) \#_{k}\left(y \#_{n} y^{\prime}\right)=\left(x \#_{k} y\right) \#_{n}\left(x^{\prime} \#_{k} y^{\prime}\right) .
$$

A functor $f: X \rightarrow Y$ of partial $\omega$-categories is a morphism of the underlying reflexive $\omega$-graphs such that, for all cells $x, y$ in $X$, if $x \#_{n} y$ is defined in $X$ then $f(x) \#_{n} f(y)$ is defined and equal to $f\left(x \#_{n} y\right)$ in $Y$. Partial $\omega$-categories and their functors form a category $\mathbf{p} \omega \mathbf{C a t}$.

1.29. We will generally confuse the notation for a $k$-cell and the units on it: for example, if $x$ is an $n$-cell and $y$ a $k$-cell, $k<n$, such that $x \#_{m} \varepsilon_{n} y$ is defined, we will write $x \#_{m} y:=x \#_{m} \varepsilon_{n} y$. 
1.30 ( $\omega$-Precategory). An $\omega$-precategory is a partial $\omega$-category $X$ such that, for all $n$-cells $x, y$ in $X$, the $k$-composition $x \#_{k} y$ is defined if and only if $\partial_{k}^{+} x=\partial_{k}^{-} y$ and $\min \{\operatorname{rk}(x), \operatorname{rk}(y)\} \leq k+1$. With their functors, $\omega$-precategories form a category $\omega$ PreCat.

1.31 ( $\omega$-Category). An $\omega$-category is a partial $\omega$-category such that, for all $n$-cells $x, y$ in $X$, the $k$-composition $x \#_{k} y$ is defined if and only if $\partial_{k}^{+} x=\partial_{k}^{-} y$. With their functors, $\omega$-categories form a category $\omega$ Cat.

1.32. The inclusion $\omega \mathbf{C a t} \hookrightarrow \mathbf{p} \omega \mathbf{C a t}$ has a left adjoint $-{ }^{*}: \mathbf{p} \omega \mathbf{C a t} \rightarrow \omega \mathbf{C a t}$; if $X$ is a partial $\omega$-category, then $X^{*}$ is the free $\omega$-category on the underlying reflexive $\omega$-graph of $X$, quotiented by all the equations involving compositions that are defined in $X$.

By [Had20b, Proposition 1.23], if $P$ is a regular directed complex, there is a partial $\omega$-category $\mathcal{M} o \ell P$ where

1. the set $\mathcal{M o l} P_{n}$ of $n$-cells is the set of molecules $U \subseteq P$ with $\operatorname{dim}(U) \leq n$,

2. $\partial_{k}^{\alpha}: \mathcal{M o l} P_{n} \rightarrow \mathcal{M o \ell} P_{k}$ is $U \mapsto \partial_{k}^{\alpha} U$,

3. $\varepsilon_{n}: \mathcal{M o l} P_{k} \rightarrow \mathcal{M o l} P_{n}$ is $U \mapsto U$,

4. $U \#_{k} V$ is defined if and only if $U \cap V=\partial_{k}^{+} U=\partial_{k}^{-} V$, and in that case it is equal to $U \cup V$.

We will write $W=U \#_{k} V$ to indicate that $W$ is a molecule decomposing as $U \cup V$, where $U$ and $V$ are molecules with $U \cap V=\partial_{k}^{+} U=\partial_{k}^{-} V$.

As detailed in [Section 7, ibid.], the assignment $P \mapsto \mathcal{M} o \ell P^{*}$ extends to a functor $\mathcal{M o \ell - *}: \mathbf{D C} \mathbf{p x}^{\mathcal{R}} \rightarrow \omega \mathbf{C a t}$ which is faithful and injective on objects.

1.33 (Principal composition). Let $x, y$ be $n$-cells in an $\omega$-precategory or an $\omega$-category, and let $k:=\min \{\operatorname{rk}(x), \operatorname{rk}(y)\}-1$. If $\partial_{k}^{+} x=\partial_{k}^{-} y$, the principal composition of $x$ and $y$ is

$$
x \# y:=x \#_{k} y \text {. }
$$

Comment 1.34. Given cells $x, y$ in an $\omega$-precategory, suppose that $x \#_{k} y$ is defined. Then either

- $\min \{\operatorname{rk}(x), \operatorname{rk}(y)\}=k+1$, in which case $x \#_{k} y=x \# y$, or

- $\operatorname{rk}(x) \leq k$, in which case $x \#_{k} y=y$, or

- $\operatorname{rk}(y) \leq k$, in which case $x \#_{k} y=x$.

This implies that $\omega$-precategories admit an axiomatisation involving only principal compositions, at the cost of explicitly handling some corner cases in the axioms.

Moreover, the two sides of (4) can both be defined only if 
- both $x$ and $x^{\prime}$ have rank lower or equal than $k+1$, in which case (4) is equivalent to

$$
x \#_{k}\left(y \#_{n} y^{\prime}\right)=\left(x \#_{k} y\right) \#_{n}\left(x \#_{k} y^{\prime}\right),
$$

or dually

- both $y$ and $y^{\prime}$ have rank lower or equal than $k+1$, in which case (4) is equivalent to

$$
\left(x \#_{n} x^{\prime}\right) \#_{k} y=\left(x \#_{k} y\right) \#_{n}\left(x^{\prime} \#_{k} y\right) .
$$

These two observations can be used to establish an equivalence between our definition of $\omega$-precategory and the one given in [FM18, Section 4.1].

1.35. There is a forgetful functor $\mathrm{U}: \omega$ Cat $\rightarrow \omega$ PreCat which makes $x \#_{k} y$ undefined whenever $\min \{\operatorname{rk}(x), \operatorname{rk}(y)\}>k+1$.

Proposition 1.36 - The functor $\mathrm{U}: \omega$ Cat $\rightarrow \omega$ PreCat is full and faithful. Its image consists of the $\omega$-precategories satisfying

$$
\left(x \#_{k-1} \partial_{k}^{-} y\right) \#_{k}\left(\partial_{k}^{+} x \#_{k-1} y\right)=\left(\partial_{k}^{-} x \#_{k-1} y\right) \#_{k}\left(x \#_{k-1} \partial_{k}^{+} y\right)
$$

for all cells $x, y$ with $\min \{\operatorname{rk}(x), \operatorname{rk}(y)\}=k+1$ and $\partial_{k-1}^{+} x=\partial_{k-1}^{-} y$.

Proof. First of all, observe that both sides of (5) are defined in an $\omega$-precategory when $x, y$ satisfy the conditions of the statement. If this precategory is of the form $\mathrm{U} X$ for some $\omega$-category $X$, then both sides are equal to $x \#_{k-1} y$ in $X$, so (5) is satisfied.

Let $X^{\prime}$ be an $\omega$-precategory such that (5) holds for all cells in $X^{\prime}$ in the conditions of the statement. We will define an $\omega$-category $X$ such that $\mathrm{U} X=X^{\prime}$, which necessarily has the same underlying reflexive $\omega$-graph as $X^{\prime}$.

Let $x, y$ be cells such that $\partial_{k-1}^{+} x=\partial_{k-1}^{-} y$ for some $k>0$. We must define $x \#_{k-1} y$ in $X$. If $\min \{\operatorname{rk}(x), \operatorname{rk}(y)\} \leq k$, then $x \#_{k-1} y$ is defined in $X^{\prime}$, and we declare it to be the same in $X$.

Otherwise, $\min \{\operatorname{rk}(x), \operatorname{rk}(y)\}=k+1+m$ for some $m \geq 0$. If $m=0$, we define $x \#_{k-1} y$ to be equal to either side of (5). For $m>0$, observe that

$$
\min \left\{\operatorname{rk}(x), \operatorname{rk}\left(\partial_{k+m}^{\alpha} y\right)\right\}, \min \left\{\operatorname{rk}\left(\partial_{k+m}^{\alpha} x\right), \operatorname{rk}(y)\right\} \leq k+m
$$

for all $\alpha \in\{+,-\}$, but

$$
\partial_{k-1}^{+} x=\partial_{k-1}^{+}\left(\partial_{k+m}^{\alpha} x\right)=\partial_{k-1}^{-}\left(\partial_{k+m}^{\alpha} y\right)=\partial_{k-1}^{-} y .
$$

We may thus assume, inductively, that $x \#_{k-1} \partial_{k+m}^{\alpha} y$ and $\partial_{k+m}^{\alpha} x \#_{k-1} y$ have already been defined, and let

$$
x \#_{k-1} y:=\left(x \#_{k-1} \partial_{k+m}^{-} y\right) \#_{k+m}\left(\partial_{k+m}^{+} x \#_{k-1} y\right) .
$$


It is an exercise to show, by induction, that this is equal to

$$
\left(\partial_{k+m}^{-} x \#_{k-1} y\right) \#_{k+m}\left(x \#_{k-1} \partial_{k+m}^{+} y\right)
$$

and derive that $X$ is an $\omega$-category. Because all the definitions are enforced by the axioms of $\omega$-categories, $X$ is unique with the property that $\mathrm{U} X=X^{\prime}$.

Since all compositions in $X$ are defined in terms of compositions in $\mathrm{U} X$, every functor $f^{\prime}: \mathrm{U} X \rightarrow \mathrm{U} Y$ of $\omega$-precategories lifts to a functor $f: X \rightarrow Y$ of $\omega$-categories. This proves fullness; faithfulness is immediate from the fact that $f$ and $\mathrm{U} f$ have the same underlying morphism of reflexive $\omega$-graphs.

1.37 (Skeleta). Let $X$ be an $\omega$-(pre)category, $n \in \mathbb{N}$. The $n$-skeleton $\sigma_{\leq n} X$ of $X$ is the restriction of $X$ to cells of rank $\leq n$. We let $\sigma_{\leq-1} X:=\emptyset$, the initial $\omega$-(pre)category. The $n$-skeleton operation extends functorially to morphisms in the obvious way.

1.38 ( $n$-Category). An $\omega$-(pre)category is an $n$-(pre)category if it is equal to its $n$-skeleton. An $n$-(pre)category is determined by its restriction to $k$-cells with $k \leq n$.

Let $n$ PreCat denote the full subcategory of $\omega$ PreCat and $n$ Cat the full subcategory of $\omega$ Cat on the $n$-(pre)categories. In both cases, the inclusion of subcategories has a right adjoint and $\sigma_{\leq n}$ is the comonad induced by the adjunction.

The inclusion also has a left adjoint, inducing a monad $\tau_{\leq n}$ : given $X$, the $n$-(pre)category $\tau_{\leq n} X$ is obtained from $\sigma_{\leq n} X$ by identifying all pairs of $n$-cells $x, y$ such that there exists an $(n+1)$-cell $e: x \Rightarrow y$ in $X$.

Remark 1.39. Both $\omega$ PreCat and $\omega$ Cat are categories of algebras for finitary monads on $\omega \mathbf{G p h}_{r e f}$, a presheaf topos. By the Remark at the end of [AR94, §2.78], they are locally finitely presentable, and in particular have all small limits and colimits. The same applies to their reflective subcategories $n$ PreCat and $n$ Cat for all $n \in \mathbb{N}$.

1.40 (Polygraph). Let $\partial O^{n}:=\sigma_{\leq n-1} O^{n}$.

A (pre)polygraph is an $\omega$-(pre)category $X$ together with a set $\mathscr{X}=\sum_{n \in \mathbb{N}} \mathscr{X}_{n}$ of generating cells such that, for all $n \in \mathbb{N}$,

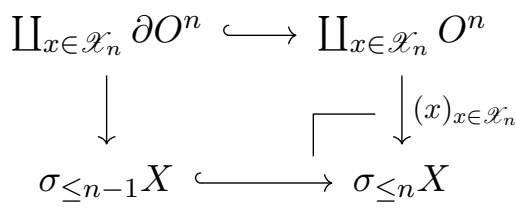

is a pushout in $\omega$ PreCat or $\omega$ Cat. 
An $n$-(pre)polygraph is a (pre)polygraph whose underlying $\omega$-(pre)category is an $n$-(pre)category. In an $n$-(pre)polygraph, $\mathscr{X}_{m}=\emptyset$ for $m>n$.

Remark 1.41. In every (pre)polygraph $(X, \mathscr{X})$, all cells in $\mathscr{X}_{n}$ have rank $n$. The set $\mathscr{X}_{0}$ is the entire set $X_{0}$ of 0 -cells.

Example 1.42. Both 1-precategories and 1-categories coincide with small categories; a 1-(pre)polygraph is a category free on a graph.

\section{MONOIDAL THEORIES}

\subsection{Planar monoidal theories}

For us, monoidal theories are embodied by pros. For technical reasons, we treat these as a special case of a more general structure of bicoloured pro, whose relation to pros is the same as the relation of bicategories to monoidal categories.

2.1 (Bicoloured pro). A bicoloured pro is a 2-category $T$ together with the structure of a 1-polygraph $\left(\sigma_{\leq 1} T, \mathscr{T}\right)$ on its 1-skeleton.

A morphism $f:(T, \mathscr{T}) \rightarrow(S, \mathscr{S})$ of bicoloured pros is a functor $f: T \rightarrow S$ of 2-categories with the property that $f(a) \in \varepsilon\left(\mathscr{S}_{0}\right) \cup \mathscr{S}_{1}$ for all $a \in \mathscr{T}_{1}$. Bicoloured pros and their morphisms form a category Pro $_{b i}$.

2.2 (Strict monoidal category). A strict monoidal category is a 2-category with a single 0 -cell.

Comment 2.3. We are using the characterisation of strict monoidal categories in [CG07, Theorem 4.1] as a definition.

2.4. When some object has a single 0-cell, we denote that 0 -cell by

2.5 (Pro). A pro is a bicoloured pro with a single 0-cell. We let Pro denote the full subcategory of $\mathbf{P r o}_{b i}$ on pros.

Comment 2.6. That is, a pro is a bicoloured pro whose underlying 2-category is a strict monoidal category.

Comment 2.7. Equivalently, a bicoloured pro $(T, \mathscr{T})$ is a 2-category whose 1-cells of type $x \Rightarrow y$ are finite paths from $x$ to $y$ in the graph

$$
\mathscr{T}_{0} \underset{\partial^{-}}{\stackrel{\partial^{+}}{\longleftarrow}} \mathscr{T}_{1}
$$


When $x=y$, the path is allowed to be of length 0 , in which case it is interpreted as the unit $\varepsilon x$.

If $T$ has a single 0 -cell, these are the same as finite ordered lists of elements of $\mathscr{T}_{1}$, that is, elements of the free monoid on $\mathscr{T}_{1}$. Seeing a pro as the embodiment of a monoidal theory, we interpret the elements of $\mathscr{T}_{1}$ as sorts, and 2-cells

$$
\varphi:\left(a_{1}, \ldots, a_{n}\right) \Rightarrow\left(b_{1}, \ldots, b_{m}\right)
$$

as operations taking $n$ inputs of sorts $a_{1}, \ldots, a_{n}$ and returning $m$ outputs of sorts $b_{1}, \ldots, b_{m}$.

In particular, if the monoidal theory is one-sorted, then $\mathscr{T}_{1}$ is a singleton, the 1-cells of $T$ are in bijection with natural numbers, and the type of a 2-cell is fixed by the arity of its input and its output. In that case, we may write $\varphi:(n) \Rightarrow(m)$ for an operation with $n$ inputs and $m$ outputs.

If we forget the structure of 1-polygraph on a pro, we get a strict monoidal category $T$, which we may see as a special kind of monoidal category. Given a monoidal category $\mathbf{M}$, a model in $\mathbf{M}$ of the monoidal theory $(T, \mathscr{T})$ is a strong monoidal functor from $T$ to $\mathbf{M}$.

Remark 2.8. Our definition of a morphism of pros allows a sort to be "collapsed" by mapping it onto a unit. We make this choice for technical reasons, even though it seems more common to disallow it, as done in [HR15]. This choice does not have any impact on models.

Example 2.9. The monoid $\mathbb{N}$ of natural numbers with addition, seen as a strict monoidal category with no rank-2 cells, is a one-sorted pro. This corresponds to the "trivial" theory of objects in a monoidal category.

Example 2.10. There is a one-sorted pro Mon whose 1-cell $(n)$ is identified with the finite ordinal $\{0<\ldots<n-1\}$ for each $n \in \mathbb{N}$, and 2-cells $\varphi:(n) \Rightarrow(m)$ are order-preserving maps. The 0-composite of $\varphi:(n) \Rightarrow(m)$ and $\psi:(p) \Rightarrow(q)$ is given by "concatenation", that is,

$$
\varphi_{\#_{0}} \psi:(n+p) \Rightarrow(m+q), \quad k \mapsto \begin{cases}\varphi(k) & \text { if } k<n, \\ m+\psi(k-n) & \text { if } k \geq n .\end{cases}
$$

This corresponds to the theory of monoids.

Example 2.11. If $(T, \mathscr{T})$ is a pro, then $\left(T^{\mathrm{co}}, \mathscr{T}^{\mathrm{co}}\right)$, obtained by reversing the orientation of all 2-cells of $T$, is also a pro. For example, $M o n^{\mathrm{co}}$ is the theory of comonoids.

Example 2.12. Let Bimod be the strict monoidal category whose 1-cells are injective maps $\imath:(k) \hookrightarrow(n)$ in Mon, 2-cells $\varphi:(\imath:(k) \hookrightarrow(n)) \Rightarrow(j:(k) \hookrightarrow(m))$ 
are commutative triangles

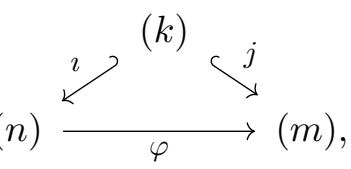

and the 0-composite of a pair of 2-cells

$$
\begin{aligned}
& \varphi:(\imath:(k) \hookrightarrow(n)) \Rightarrow(j:(k) \hookrightarrow(m)), \\
& \psi:\left(\imath^{\prime}:(\ell) \hookrightarrow(p)\right) \Rightarrow\left(j^{\prime}:(\ell) \hookrightarrow(q)\right)
\end{aligned}
$$

is the commutative triangle

$$
(n+p) \underset{\varphi \#_{0} \psi}{\longrightarrow}(m+q) .
$$

The 1-cells in Bimod are freely generated under 0-composition by the pair

$$
\imath:(0) \hookrightarrow(1), \quad \mathrm{id}_{1}:(1) \rightarrow(1),
$$

where $\imath$ is the unique inclusion of the empty ordinal, so Bimod admits the structure of a two-sorted pro.

There are morphisms of pros Mon $\rightarrow \operatorname{Bimod}$ and $\mathbb{N} \rightarrow \operatorname{Bimod}$, sending the generating 1-cell to $\imath$ and $\operatorname{id}_{1}$, respectively. The models of Bimod in a monoidal category $\mathbf{M}$ are given by an object of $\mathbf{M}$, a monoid in $\mathbf{M}$, and a two-sided action of the monoid on the object, making the object a bimodule.

2.13. There is an obvious functor U: $\mathbf{P r o}_{b i} \rightarrow 2$ Cat forgetting the structure of 1-polygraph. This functor has a right adjoint R: $2 \mathbf{C a t} \rightarrow \mathbf{P r o}_{b i}$, described as follows.

Given a 2-category $X$, the 1-skeleton of $\mathrm{R} X$ is free on the underlying reflexive graph of $\sigma_{\leq 1} X$ : that is, the sorts of $\mathrm{R} X$ are all the 1-cells of rank 1 in $X$. The 2-cells of type $\left(a_{1}, \ldots, a_{n}\right) \Rightarrow\left(b_{1}, \ldots, b_{m}\right)$ in $\mathrm{R} X$ are the 2-cells of type $a_{1} \#_{0} \ldots \#_{0} a_{n} \Rightarrow b_{1} \#_{0} \ldots \#_{0} b_{m}$ in $X$.

Compositions are induced by those of $X$ in the obvious way, and a functor $f: X \rightarrow Y$ of 2-categories induces a morphism $\mathrm{R} f: \mathrm{R} X \rightarrow \mathrm{R} Y$ of bicoloured pros by

$$
\begin{aligned}
\varphi: & \left(a_{1}, \ldots, a_{n}\right) \Rightarrow\left(b_{1}, \ldots, b_{m}\right) \\
\mapsto f(\varphi): & f\left(a_{1}\right) \#_{0} \ldots \#_{0} f\left(a_{n}\right) \Rightarrow f\left(b_{1}\right) \#_{0} \ldots \#_{0} f\left(b_{m}\right) .
\end{aligned}
$$


For each 2-cell $\varphi$ of type $\left(a_{1}, \ldots, a_{n}\right) \Rightarrow\left(b_{1}, \ldots, b_{m}\right)$ in a bicoloured pro $(T, \mathscr{T})$, there is a 2-cell $a_{1} \#_{0} \ldots \#_{0} a_{n} \Rightarrow b_{1} \#_{0} \ldots \#_{0} b_{m}$ in $\mathrm{U}(T, \mathscr{T})$, which in turn induces a 2 -cell of type $\left(a_{1}, \ldots, a_{n}\right) \Rightarrow\left(b_{1}, \ldots, b_{m}\right)$ in $\mathrm{RU}(T, \mathscr{T})$; the unit of the adjunction sends $\varphi$ to this 2-cell.

Conversely, for each 2-category $X$ and 2-cell $\varphi:\left(a_{1}, \ldots, a_{n}\right) \Rightarrow\left(b_{1}, \ldots, b_{m}\right)$ in URX, the counit sends $\varphi$ to the cell of type $a_{1} \#_{0} \ldots \#_{0} a_{n} \Rightarrow b_{1} \#_{0} \ldots \#_{0} b_{m}$ in $X$ from which it was induced. It is an exercise to show that the unit and counit satisfy the required equations and determine an adjunction.

Lemma 2.14 - The category Pro $_{b i}$ has equalisers.

Proof. Let $f, g:(T, \mathscr{T}) \rightarrow(S, \mathscr{S})$ be parallel morphisms of bicoloured pros. Define $T^{\prime}$ to be the restriction of $T$ to the cells $x$ that satisfy $f(x)=g(x)$ in $S$. Then $T^{\prime}$ is a 2-category.

A 1-cell $\left(a_{1}, \ldots, a_{n}\right)$ in $T$ belongs to $T^{\prime}$ if and only if $f\left(a_{i}\right)=g\left(a_{i}\right)$ for all $i \in\{1, \ldots, n\}$. It follows that $\mathscr{T}^{\prime}:=\{a \in \mathscr{T} \mid f(a)=g(a)\}$ gives $\sigma_{\leq 1} T^{\prime}$ the structure of a 1-polygraph, so that the inclusion of $T^{\prime}$ into $T$ is a morphism of bicoloured pros. By a routine argument, it is the equaliser of $f$ and $g$.

Proposition 2.15 - The functor U: Pro ${ }_{b i} \rightarrow 2$ Cat is comonadic.

Proof. We have shown that $\mathrm{U}$ has a right adjoint. Moreover, equalisers as constructed in the proof of Lemma 2.14 are evidently created by $\mathrm{U}$.

In order to apply the dual of Beck's monadicity theorem [ML71, §VI.7], it suffices to show that $\mathrm{U}$ reflects isomorphisms. Let $f:(T, \mathscr{T}) \rightarrow(S, \mathscr{S})$ be a morphism of bicoloured pros and suppose that $\mathrm{U} f$ is an isomorphism of 2-categories with inverse $g$. Then both $\cup f$ and $g$ must preserve the rank of all cells.

Let $a \in \mathscr{S}_{1}$. Then $g(a)$ can be written uniquely as a finite path $\left(a_{1}, \ldots, a_{n}\right)$ with $a_{i} \in \mathscr{T}_{1}$ for all $i \in\{1, \ldots, n\}$, and

$$
a=\bigcup f(g(a))=\left(f\left(a_{1}\right), \ldots, f\left(a_{n}\right)\right)
$$

where $n>0$ and $f\left(a_{i}\right) \in \mathscr{S}_{1}$ for all $i \in\{1, \ldots, n\}$. Because $\sigma_{\leq 1} S$ is free, this is only possible if $n=1$ and $f\left(a_{1}\right)=a$. It follows that $g$ sends generators to generators, hence it determines a morphism of bicoloured pros, inverse to $f$ in Pro $_{b i}$.

Corollary 2.16 - The categories Pro $_{b i}$ and Pro have all small limits and colimits.

Proof. By the dual of [ML71, Exercise 2, §VI.2], a comonadic functor creates all colimits in its codomain; since 2 Cat has all small colimits, so does Pro ${ }_{b i}$. 
Moreover, 2Cat has all small limits and Pro $\mathbf{P r}_{b i}$ has equalisers, so Pro $\mathbf{P r}_{b i}$ has all small limits by the dual of [Lin69, Corollary 2].

Since Pro is defined equationally, it is a reflective subcategory of $\mathbf{P r o}_{b i}$. It follows from [Rie17, Proposition 4.5.15] that it also has all small limits and colimits.

\subsection{Non-planar monoidal theories}

2.17 (Braided strict monoidal category). A braided strict monoidal category is a strict monoidal category $X$ together with a family of 2-cells

$$
\sigma_{x, y}: x \#_{0} y \Rightarrow y \#_{0} x
$$

called braidings, indexed by 1-cells $x, y$, satisfying the following axioms:

1. the braidings are invertible, that is, there are unique 2-cells $\sigma_{x, y}^{-1}$, called inverse braidings, such that $\sigma_{x, y} \#_{1} \sigma_{x, y}^{-1}$ and $\sigma_{x, y}^{-1} \#_{1} \sigma_{x, y}$ are units;

2. they are natural in their parameters, that is, for all 2-cells $\varphi: x \Rightarrow x^{\prime}$ and $\psi: y \Rightarrow y^{\prime}$

$$
\begin{aligned}
& \left(\varphi \#_{0} y\right) \#_{1} \sigma_{x^{\prime}, y}=\sigma_{x, y} \#_{1}\left(y \#_{0} \varphi\right), \\
& \left(x \#_{0} \psi\right) \#_{1} \sigma_{x, y^{\prime}}=\sigma_{x, y} \#_{1}\left(\psi \#_{0} x\right) ;
\end{aligned}
$$

3. they are compatible with 0-composition and units, that is,

$$
\begin{aligned}
\sigma_{x \#_{0} x^{\prime}, y} & =\left(x \#_{0} \sigma_{x^{\prime}, y}\right) \#_{1}\left(\sigma_{x, y} \#_{0} x^{\prime}\right), \\
\sigma_{x, y \#_{0} y^{\prime}} & =\left(\sigma_{x, y} \#_{0} y^{\prime}\right) \#_{1}\left(y \#_{0} \sigma_{x, y^{\prime}}\right), \\
\sigma_{\varepsilon \bullet, y} & =\varepsilon y, \quad \sigma_{x, \varepsilon} \bullet \varepsilon x,
\end{aligned}
$$

whenever the left-hand side is defined.

A functor $f: X \rightarrow Y$ of braided strict monoidal categories is a functor of the underlying 2-categories that preserves braidings, that is, $f\left(\sigma_{x, y}\right)=\sigma_{f(x), f(y)}$ for all 1-cells $x, y$ in $X$. With their functors, braided strict monoidal categories form a category BrMonCat $_{s t r}$.

2.18 (Prob). A prob is a pro together with a structure of braided strict monoidal category on its underlying strict monoidal category.

A morphism of probs is a morphism of pros that preserves the braidings. Probs and their morphisms form a category Prob.

Comment 2.19. Models of probs live in braided monoidal categories $\mathbf{M}$, not necessarily strict. A model of a prob $(T, \mathscr{T})$ in $\mathbf{M}$ is a braided strong monoidal functor from $T$ to $\mathbf{M}$. 
Remark 2.20. To determine a unique structure of braided strict monoidal category on a pro it is, in fact, sufficient to give braidings $\sigma_{a, b}$ for all pairs of generating 1-cells $a, b$; a morphism of pros that preserves these braidings automatically preserves all braidings. This is a consequence of axiom 3 of braided strict monoidal categories, since every 1-cell in a pro can be decomposed as a composite of generating 1-cells.

2.21 (Dual braided structure). Let $X$ be a braided strict monoidal category with braidings $\left\{\sigma_{x, y}\right\}$. The family of 2-cells

$$
\sigma_{x, y}^{*}:=\sigma_{y, x}^{-1}
$$

defines a second structure $X^{*}$ of braided strict monoidal category on the underlying strict monoidal category of $X$.

If $f: X \rightarrow Y$ is a functor of braided strict monoidal categories, the same underlying functor of 2-categories determines a functor $f^{*}: X^{*} \rightarrow Y^{*}$. This defines an involution $-{ }^{*}$ on BrMonCat Btr $_{\text {, }}$, which also induces a duality on Prob.

2.22 (Symmetric strict monoidal category). A symmetric strict monoidal category is a braided strict monoidal category $X$ satisfying $X=X^{*}$.

2.23 (Prop). A prop is a prob whose underlying braided strict monoidal category is symmetric. We let Prop denote the full subcategory of Prob on props.

2.24. There is an obvious forgetful functor $U:$ Prob $\rightarrow$ Pro and an inclusion of subcategories Prop $\hookrightarrow$ Prob. Both of these have left adjoints:

- the left adjoint F: Pro $\rightarrow$ Prob of $\mathrm{U}$ freely adds braidings $\sigma_{x, y}$ and inverse braidings $\sigma_{x, y}^{-1}$ for all pairs of 1-cells $x, y$ of a pro (or just the generating ones, see Remark 2.20), then quotients by the axioms of braided strict monoidal categories;

- the reflector $\mathrm{r}:$ Prob $\rightarrow$ Prop quotients by the equation $\sigma_{x, y}=\sigma_{x, y}^{*}$ for all pairs of 1-cells $x, y$ of a prob.

Since we have not yet shown that Prob has coequalisers, for the moment we can interpret the latter as a coequaliser in Pro, then observe that the images of the $\sigma_{x, y}$ still form a family of braidings in the quotient.

Example 2.25. The free prob $\mathbb{B}:=\mathrm{FN}$ is the theory of braids. With 1-composition, 2-cells of type $(n) \Rightarrow(n)$ in $\mathbb{B}$ form the braid group $B_{n}$ on $n$ strands. 
Example 2.26. The prop reflection $\mathbb{S}:=\mathrm{r} \mathbb{B}$ of the theory of braids is the theory of permutations. With 1-composition, 2-cells of type $(n) \Rightarrow(n)$ in $\mathbb{S}$ form the symmetric group $S_{n}$ on $n$ elements.

Example 2.27. Let CMon be defined as Mon, but 2-cells of type $(n) \Rightarrow(m)$ are all functions from $(n)$ to $(m)$, not just the order-preserving ones. This is a one-sorted prop with braidings generated by

$$
\sigma_{1,1}:(2) \Rightarrow(2), \quad 0 \mapsto 1,1 \mapsto 0 .
$$

It corresponds to the theory of commutative monoids in symmetric monoidal categories. Similarly, models of $\mathrm{CMon}^{\mathrm{co}}$ are commutative comonoids.

Example 2.28. There is a one-sorted pro $M a t_{\mathbb{Z}}$ whose 2-cells $A:(n) \Rightarrow(m)$ are $(m \times n)$-matrices of integers, the 1 -composite $A \#_{1} B$ is the product $B A$ of matrices, and

$$
A \#_{0} B:=\left(\begin{array}{cc}
A & 0 \\
0 & B
\end{array}\right) .
$$

This is a prop with braidings generated by

$$
\sigma_{1,1}:=\left(\begin{array}{ll}
0 & 1 \\
1 & 0
\end{array}\right)
$$

There is a morphism of props $C M o n \rightarrow \operatorname{Mat}_{\mathbb{Z}}$ sending $\varphi:(n) \Rightarrow(m)$ to the $(m \times n)$-matrix $A_{\varphi}$ with entries

$$
A_{\varphi}(j, i):= \begin{cases}1 & \text { if } j=\varphi(i), \\ 0 & \text { otherwise }\end{cases}
$$

and a morphism $\mathrm{CMon}^{\mathrm{co}} \rightarrow$ Mat $_{\mathbb{Z}}$ sending $\varphi^{\mathrm{co}}:(m) \Rightarrow(n)$ to the transpose of $A_{\varphi}$. As a symmetric monoidal theory, $M a t_{\mathbb{Z}}$ corresponds to the theory of commutative and cocommutative Hopf algebras [BSZ17, Section 7].

2.29 (Gray-category). A Gray-category is a 3-precategory $G$ together with a family of 3-cells

$$
\chi_{x, y}:\left(x \#_{0} \partial^{-} y\right) \#_{1}\left(\partial^{+} x \#_{0} y\right) \Rightarrow\left(\partial^{-} x \#_{0} y\right) \#_{1}\left(x \#_{0} \partial^{+} y\right)
$$

called interchangers, indexed by 2-cells $x, y$ with $\partial_{0}^{+} x=\partial_{0}^{-} y$, satisfying the following axioms:

1. the interchangers are invertible, that is, there are unique 3 -cells

$$
\chi_{x, y}^{-1}:\left(\partial^{-} x \#_{0} y\right) \#_{1}\left(x \#_{0} \partial^{+} y\right) \Rightarrow\left(x \#_{0} \partial^{-} y\right) \#_{1}\left(\partial^{+} x \#_{0} y\right)
$$

called inverse interchangers, such that $\chi_{x, y} \#_{2} \chi_{x, y}^{-1}$ and $\chi_{x, y}^{-1} \#_{2} \chi_{x, y}$ are units; 
2. the interchangers are natural in their parameters, that is, for all 3-cells $\varphi: x \Rightarrow x^{\prime}$ and $\psi: y \Rightarrow y^{\prime}$ with $\partial_{0}^{+} \varphi=\partial_{0}^{-} \psi$,

$$
\begin{aligned}
& \left(\left(\varphi \#_{0} \partial^{-} y\right) \#_{1}\left(\partial_{1}^{+} \varphi \#_{0} y\right)\right) \#_{2} \chi_{x^{\prime}, y}=\chi_{x, y} \#_{2}\left(\left(\partial_{1}^{-} \varphi \#_{0} y\right) \#_{1}\left(\varphi \#_{0} \partial^{+} y\right)\right), \\
& \left(\left(x \#_{0} \partial_{1}^{-} \psi\right) \#_{1}\left(\partial^{+} x \#_{0} \psi\right)\right) \#_{2} \chi_{x, y^{\prime}}=\chi_{x, y} \#_{2}\left(\left(\partial^{-} x \#_{0} \psi\right) \#_{1}\left(x \#_{0} \partial_{1}^{+} \psi\right)\right) ;
\end{aligned}
$$

3. the interchangers are compatible with 1-compositions and units, that is,

$$
\begin{aligned}
\chi_{x \#_{1} x^{\prime}, y} & =\left(\left(x \#_{0} \partial^{-} y\right) \#_{1} \chi_{x^{\prime}, y}\right) \#_{2}\left(\chi_{x, y} \#_{1}\left(x^{\prime} \#_{0} \partial^{+} y\right)\right), \\
\chi_{x, y \#_{1} y^{\prime}} & \left.=\left(\chi_{x, y} \#_{1}\left(\partial^{+} x \#_{0} y^{\prime}\right)\right) \#_{2}\left(\left(\partial^{-} x \#_{0} y\right) \#_{1} \chi_{x, y^{\prime}}\right)\right), \\
\chi_{\varepsilon x, y} & =\varepsilon\left(\varepsilon x \#_{0} y\right), \quad \chi_{x, \varepsilon y}=\varepsilon\left(x \#_{0} \varepsilon y\right),
\end{aligned}
$$

whenever the left-hand side is defined;

4. for all pairs of 3 -cells $\varphi, \psi$ with $\partial_{1}^{+} \varphi=\partial_{1}^{-} \psi$, the equation

$$
\left(\varphi \#_{1} \partial_{2}^{-} \psi\right) \#_{2}\left(\partial_{2}^{+} \varphi \#_{1} \psi\right)=\left(\partial_{2}^{-} \varphi \#_{1} \psi\right) \#_{2}\left(\varphi \#_{1} \partial_{2}^{+} \psi\right)
$$

holds in $G$.

A functor $f: G \rightarrow H$ of Gray-categories is a functor of the underlying 3-precategories that preserves the interchangers, that is, $f\left(\chi_{x, y}\right)=\chi_{f(x), f(y)}$ for all suitable 2-cells $x, y$ in $G$. With their functors, Gray-categories form a category GrayCat.

Remark 2.30. Axiom 4 is an instance of (5), allowing us to univocally define the 1-composition $\varphi \#_{1} \psi$ of 3-cells with $\partial_{1}^{+} \varphi=\partial_{1}^{-} \psi$ in a Gray-category.

Comment 2.31. A more concise definition is that a Gray-category is a small category enriched over 2Cat with the "pseudo" Gray tensor product [GPS95, Chapter 5]. As in [Lac11, §1.4], one derives that GrayCat is locally finitely presentable, and in particular has all small limits and colimits.

2.32. By Proposition 1.36, every 3-category seen as a 3-precategory admits a natural structure of Gray-category with units as interchangers. This defines an embedding 3Cat $\hookrightarrow$ GrayCat, which makes 3Cat a reflective subcategory of GrayCat: the reflector universally turns the interchangers into units.

2.33. Given a braided strict monoidal category $X$, we define a Gray-category $\mathrm{B} X$ as follows. For all $n \in \mathbb{N}$, we let $\mathrm{B} X_{n+1}:=X_{n}$, with the same boundary and unit operators as $X$ between $\mathrm{B} X_{n+2}$ and $\mathrm{B} X_{n+1}$. We let $\mathrm{B} X_{0}:=\{\bullet\}$, with the only possible unit and boundary operators relating it to $\mathrm{B} X_{1}$. This defines the underlying reflexive $\omega$-graph of $\mathrm{B} X$.

To make $\mathrm{B} X$ a 3-precategory, it suffices to define the principal compositions. Because $B X$ has no rank-1 cells, the principal compositions are of the form $x \#_{k} y$ where 
- $k=1$ and $\min \{\operatorname{rk}(x), \operatorname{rk}(y)\}=2$, or

- $k=2$ and $\operatorname{rk}(x)=\operatorname{rk}(y)=3$.

In either case, $x \#_{k-1} y$ is defined in $X$, and we let $x \#_{k} y$ be equal to it in $\mathrm{B} X$.

Finally, given 2-cells $x, y$ in $\mathrm{B} X$, we let the interchanger $\chi_{x, y}$ correspond to the braiding $\sigma_{x, y}$ in $X$. It is an exercise to check that this gives $\mathrm{B} X$ the structure of a Gray-category.

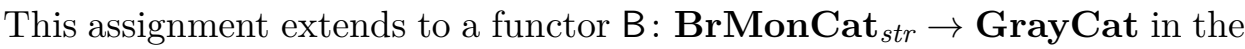
obvious way. By [CG11, Theorem 2.16], this functor is full and faithful, and its essential image consists exactly of those Gray-categories that have a single 0 -cell and a single 1-cell. This can be seen as an alternative characterisation

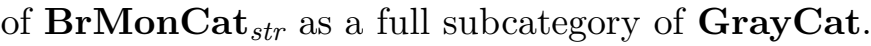

Remark 2.34. Through B, the duality -* on BrMonCat str $_{\text {is }}$ is the restriction of the duality on GrayCat that reverses the orientation of 1-cells.

2.35. If $X$ is a braided strict monoidal category, the structure of a 1-polygraph on $\sigma_{\leq 1} X$ determines a unique structure of 2-prepolygraph on $\sigma_{\leq 2} \mathrm{~B} X$, and vice versa. A functor $f$ of braided strict monoidal categories sends generators to generators if and only if $\mathrm{B} f$ does.

Thus, a prob is equivalently defined as a Gray-category $T$ with a single 0 -cell, a single 1-cell, and the structure of a 2-prepolygraph $\left(\sigma_{\leq 2} T, \mathscr{T}\right)$ on its 2-skeleton. A morphism $f:(T, \mathscr{T}) \rightarrow(S, \mathscr{S})$ of probs is a functor of Gray-categories such that $f(a) \in\left\{\varepsilon_{2} \bullet\right\} \cup \mathscr{S}_{2}$ for all $a \in \mathscr{T}_{2}$.

We conclude that there is a triangle of functors

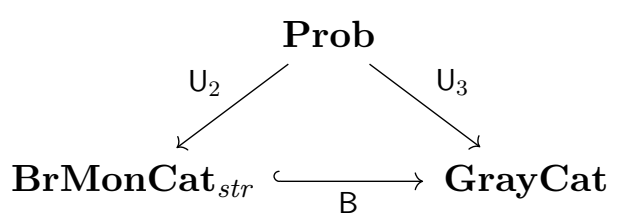

commuting up to natural isomorphism, where $\mathrm{U}_{2}$ and $\mathrm{U}_{3}$ are the forgetful functors associated to the two alternative definitions of prob.

Proposition 2.36 - The categories BrMonCat Btr $_{\text {, }}$ Prob, and Prop have all small limits and colimits.

Sketch of the proof. First of all, the essential image of B is defined equationally in GrayCat, so BrMonCat str $_{\text {is, }}$ up to equivalence, a reflective subcategory. Since GrayCat has all small limits and colimits, by [Rie17, Proposition 4.5.15] so does BrMonCat str. $_{\text {. }}$.

Then, we can mimic the proofs of Lemma 2.14 and Proposition 2.15 to show that Prob has equalisers and that the functor $\mathrm{U}_{2}$ : Prob $\rightarrow$ BrMonCat $_{s t r}$ is 
comonadic. As in the proof of Corollary 2.16, we deduce that Prob has all small limits and colimits, and so does its reflective subcategory Prop.

Remark 2.37. The functors U: Pro ${ }_{b i} \rightarrow 2$ Cat, $\mathrm{U}_{2}:$ Prob $\rightarrow$ BrMonCat $_{s t r}$ are pseudomonic: that is, in addition to being faithful, they reflect and are full on isomorphisms. This captures the fact that a 2-category admits at most one structure of bicoloured pro, a consequence of the general statement, proved by Michael Makkai [Mak05, Section 4, Proposition 8], that an $\omega$-category admits at most one structure of polygraph.

Because the composite of a pseudomonic with a full and faithful functor is pseudomonic, it follows that $\mathrm{U}_{3}:$ Prob $\rightarrow$ GrayCat is also pseudomonic.

\section{COMBinatorial RESUlts}

\subsection{In generic dimension}

In this section, we use results of [Had20a, Section 6]. These results are stated relative to the restricted class of constructible directed complexes, but the proofs do not involve any properties that are not satisfied by all regular directed complexes. Thus all cited statements hold with constructible replaced by regular.

3.1. Let $U$ be a closed subset of a regular directed complex. For each $n \geq-1$, the bipartite directed graph $\mathscr{M}_{n} U$ has

$$
\{x \in U \mid \operatorname{dim}(x) \leq n\}+\{x \in U \mid x \text { is maximal and } \operatorname{dim}(x)>n\}
$$

as set of vertices, and an edge $y \rightarrow x$ if and only if

- $\operatorname{dim}(y) \leq n, \operatorname{dim}(x)>n$, and $y \in \partial_{n}^{-} x \backslash \partial_{n-1} x$, or

- $\operatorname{dim}(y)>n, \operatorname{dim}(x) \leq n$, and $x \in \partial_{n}^{+} y \backslash \partial_{n-1} y$.

3.2 (Frame dimension). Let $U$ be a closed subset of a regular directed complex. The frame dimension of $U$ is the integer

$$
\operatorname{frdim}(U):=\max \{\operatorname{dim}(\operatorname{cl}\{x\} \cap \operatorname{cl}\{y\}) \mid x, y \text { maximal in } U, x \neq y\} .
$$

Remark 3.3. If $\operatorname{frdim}(U)=-1$, then $U$ is a disjoint union of atoms.

3.4 (Frame acyclicity). A regular directed complex $P$ is frame-acyclic if, for all molecules $U$ in $P$, if $\operatorname{frdim}(U)=k$, then $\mathscr{M}_{k} U$ is acyclic.

Lemma 3.5 - Let $P$ be a frame-acyclic regular directed complex. Then 
1. for all molecules $U$ in $P$, if $U=U_{1} \cup U_{2}$ for some closed subsets $U_{1}, U_{2}$ such that $U_{1} \cap U_{2}=\partial_{k}^{+} U_{1}=\partial_{k}^{-} U_{2}$, then $U_{1}$ and $U_{2}$ are molecules;

2. $\left(\mathcal{M o \ell} P^{*},\{\operatorname{cl}\{x\}\}_{x \in P}\right)$ is a polygraph.

Proof. A corollary of [Had20a, Proposition 26].

3.6 ( $k$-Order). Let $U$ be a regular $n$-molecule. For $k<n$, a $k$-order on $U$ is a linear ordering $\left(x_{1}, \ldots, x_{m}\right)$ of the set

$$
\{x \in U \mid x \text { is maximal and } \operatorname{dim}(x)>k\}
$$

with the property that, if there is a path from $x_{i}$ to $x_{j}$ in $\mathscr{M}_{k} U$, then $i \leq j$.

Proposition 3.7 - Let $U$ be a regular $n$-molecule, $k<n$. If $\mathscr{M}_{k} U$ is acyclic, then $U$ admits a k-order.

Proof. Every directed acyclic graph admits a topological sorting, that is, a linear order $\preceq$ on its vertices with the property that if there is a path from $x$ to $y$, then $x \preceq y$. The restriction of a topological sorting of $\mathscr{M}_{k} U$ to the maximal elements of dimension greater than $k$ is a $k$-order on $U$.

Proposition 3.8 - Let $U$ be a regular n-molecule. Then $\mathscr{M}_{n-1} U$ is acyclic and $U$ admits an $(n-1)$-order.

Proof. Follows from Proposition 3.7 and [Had20a, Proposition 20].

Lemma 3.9 - Let $U$ be a frame-acyclic regular molecule, $k \geq \operatorname{frdim}(U)$, and let $\left(x_{1}, \ldots, x_{m}\right)$ be a $k$-order on $U$. There exist molecules $V_{1}, \ldots, V_{m}$, such that

$$
U=V_{1} \#_{k} \ldots \#_{k} V_{m}
$$

and $x_{i} \in V_{j}$ if and only if $i=j$ for all $i, j \in\{1, \ldots m\}$.

Proof. If $m=1$ then $V_{1}:=U$ satisfies the statement. Suppose that $m>1$ and that $k=\operatorname{frdim}(U)$. Then we proceed as in the proof of [Had20a, Proposition $26]$ to produce $i \in\{1, \ldots, m-1\}$ and a decomposition $U=U_{1} \cup U_{2}$ such that

1. $U_{1}$ contains $x_{1}, \ldots, x_{i}$ and $U_{2}$ contains $x_{i+1}, \ldots, x_{m}$,

2. $U_{1} \cap U_{2}=\partial_{k}^{+} U_{1}=\partial_{k}^{-} U_{2}$.

By Lemma 3.5, both $U_{1}$ and $U_{2}$ are molecules. Moreover $\left(x_{1}, \ldots, x_{i}\right)$ and $\left(x_{i+1}, \ldots, x_{m}\right)$ are $k$-orders on $U_{1}$ and $U_{2}$, respectively. We conclude by the inductive hypothesis applied to $U_{1}$ and $U_{2}$. 
Finally, suppose that $k>\ell:=\operatorname{frdim}(U)$. By frame acyclicity, we can fix an $\ell$-order $\left(y_{1}, \ldots, y_{p}\right)$ on $U$, and by the first part of the proof we can decompose $U$ as

$$
W_{1} \#_{\ell} \ldots \#_{\ell} W_{p}
$$

with $y_{i} \in W_{j}$ if and only if $i=j$. Now for each $i \in\{1, \ldots, m\}$ there is a unique $j(i) \in\{1, \ldots, p\}$ such that $x_{i}=y_{j(i)}$. Let

$$
\begin{aligned}
V_{i} & :=\partial_{k}^{\alpha(i, 1)} W_{1} \#_{\ell} \ldots \#_{\ell} W_{j(i)} \#_{\ell} \ldots \#_{\ell} \partial_{k}^{\alpha(i, p)} W_{p}, \text { where } \\
\alpha(i, j) & := \begin{cases}+ & \text { if } j=j\left(i^{\prime}\right) \text { for some } i^{\prime}<i, \\
- & \text { otherwise. }\end{cases}
\end{aligned}
$$

Then $U=V_{1} \#_{k} \ldots \#_{k} V_{m}$ is the required decomposition.

3.10 (Substitution). Let $V$ and $W$ be regular $n$-molecules with spherical boundary, let $U$ be a regular $n$-molecule, and suppose $V \sqsubseteq U$. Then $U \backslash(V \backslash \partial V)$ is a closed subset of $U$.

Suppose that $\partial^{\alpha} V$ is isomorphic to $\partial^{\alpha} W$ for all $\alpha \in\{+,-\}$. From [Had20b, Lemma 2.2] we obtain a unique isomorphism $\imath: \partial U \stackrel{\sim}{\hookrightarrow} \partial V$. We define $U[W / V]$ to be the pushout

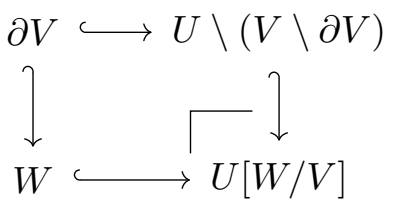

in $\mathbf{D C p x} \mathbf{x}^{\mathcal{R}}$, and call it the substitution of $W$ for $V \sqsubseteq U$. By [Proposition 2.4, ibid.] this is an $n$-molecule with boundaries isomorphic to those of $U$, and such that $W \sqsubseteq U[W / V]$.

Remark 3.11. As shown in [Had20b, Lemma 2.5], if $U$ is an $n$-molecule with a decomposition $U=V_{1} \#_{n-1} \ldots \#_{n-1} V_{m}$ as in Lemma 3.9 , then $\partial^{\alpha} V_{i}$ is isomorphic to $\partial^{-\alpha} V_{i}\left[\partial^{\alpha} x_{i} / \partial^{-\alpha} x_{i}\right]$ for all $\alpha \in\{+,-\}$ and $i \in\{1, \ldots, m\}$.

\subsection{In low dimension}

3.12 (Totally loop-free molecule). Given a regular molecule $U$, let $\mathscr{H}^{\circ} U$ be the directed graph obtained from $\mathscr{H} U$ by reversing all the edges labelled -. We say that $U$ is totally loop-free if $\mathscr{H}^{\circ} U$ is acyclic as a directed graph.

If $U$ is totally loop-free, for all $x, y \in U$, we let $x \preceq y$ if and only if there is a path from $x$ to $y$ in $U$.

Proposition 3.13 - Let $U$ be a regular molecule. If $\operatorname{dim}(U) \leq 2$, then $U$ is totally loop-free and $\preceq$ is a linear order on $U$. 
Proof. If $\operatorname{dim}(U) \in\{0,1\}$ or if $U$ is a 2-dimensional atom, this is easy. Otherwise, decompose $U$ as $V_{1} \#_{1} \ldots \#_{1} V_{m}$ so that each $V_{i}$ contains a unique 2-dimensional element $x_{i}$, and let $V_{0}:=\partial^{-} U$ and $U^{\prime}:=V_{0} \#_{1} \ldots \#_{1} V_{m-1}$. Then $U=U^{\prime} \cup \operatorname{cl}\left\{x_{m}\right\}$, and we may assume inductively that the statement holds for $U^{\prime}$.

Suppose that there is a cycle in $\mathscr{H}^{\circ} U$. Because both $U^{\prime}$ and $\operatorname{cl}\left\{x_{m}\right\}$ are totally loop-free, such a cycle must leave $U^{\prime}$, enter $\operatorname{cl}\left\{x_{m}\right\} \backslash \partial^{-} x_{m}$, then return to $U^{\prime}$. Such a path either

- enters $x_{m}$ via $\Delta^{-} x_{m}$, then enters $\partial^{+} x_{m}$ and leaves via $\partial_{0}^{+} x_{m}$, or

- enters $\Delta^{+} x_{m}$ directly via $\partial_{0}^{-} x_{m}$, stays in $\partial^{+} x_{m}$ and leaves via $\partial_{0}^{+} x_{m}$.

In both cases, the path through $\operatorname{cl}\left\{x_{m}\right\} \backslash \partial^{-} x_{m}$ can be replaced with the unique path to $\partial_{0}^{+} x_{m}$ that stays in $\partial^{-} x_{m} \subseteq U^{\prime}$. In this way, we create a cycle in $\mathscr{H}^{\circ} U^{\prime}$, contradicting the inductive hypothesis.

This proves that $U$ is totally loop-free. To show that $\preceq$ is a linear order, it suffices to compare elements of $U^{\prime}$ and of $\operatorname{cl}\left\{x_{m}\right\} \backslash \partial^{-} x_{m}$. Let $x \in U^{\prime}$. There are two possible cases:

- $\partial_{0}^{+} x_{m} \preceq x$ in $U^{\prime}$. Then $z \preceq x$ for all elements $z \in \operatorname{cl}\left\{x_{m}\right\}$.

- $x \prec \partial_{0}^{+} x_{m}$ in $U^{\prime}$. Let $y$ be the unique 1-dimensional element of $\Delta^{-} x_{m}$ that covers $\partial_{0}^{+} x_{m}$. Suppose that $y \prec x$ in $U^{\prime}$, that is, there is a non-trivial path from $y$ to $x$ in $\mathscr{H}^{\circ} U^{\prime}$. Such a path cannot pass through $\partial^{+} y=\partial_{0}^{+} x_{m}$, for otherwise $\partial_{0}^{+} x_{m} \preceq x$; nor it can enter a 2-dimensional element, because $y$ is not covered by any element of $U^{\prime}$ with orientation -. Therefore $x \preceq y$, so $x \prec x_{m}$ and $x \prec z$ for all elements $z \in \partial^{+} x_{m} \backslash \partial_{0} x_{m}$.

This proves that $\preceq$ is a linear order on $U$.

Remark 3.14. If $U$ is a regular molecule with $\operatorname{dim}(U) \leq 2$, by [Ste93, Theorem 2.17] combined with Proposition 3.13, $\mathcal{M o l U ^ { * }}$ is equal to $\mathcal{M o \ell U}$.

Proposition 3.15 - Let $U$ be a regular 2-molecule, $k \in\{0,1\}$, and let $x, y \in U$ be maximal elements of dimension $>k$. If there is a path from $x$ to $y$ in $\mathscr{M}_{k} U$, then $x \preceq y$.

Proof. Suppose $k=1$. A path $x=x_{0} \rightarrow w_{0} \rightarrow \ldots \rightarrow w_{m-1} \rightarrow x_{m}=y$ in $\mathscr{M}_{1} U$ is a concatenation of two-step paths $x_{i} \rightarrow w_{i} \rightarrow x_{i+1}$ where $\operatorname{dim}\left(x_{i}\right)=2$ for all $i \in\{0, \ldots, m\}$ and

$$
w_{i} \in\left(\partial^{+} x_{i} \backslash \partial_{0} x_{i}\right) \cap\left(\partial^{-} x_{i+1} \backslash \partial_{0} x_{i+1}\right) .
$$

If $\operatorname{dim}\left(w_{i}\right)=1$ then $w_{i} \in \Delta^{+} x_{i} \cap \Delta^{-} x_{i+1}$, so $x_{i} \prec w_{i} \prec x_{i+1}$. Suppose $\operatorname{dim}\left(w_{i}\right)=0$. Because $\partial^{+} x_{i}$ is pure and 1-dimensional, $w_{i}$ is covered by some 
element of $\Delta^{+} x_{i}$, and because $w_{i} \notin \partial_{0} x_{i}=\Delta\left(\partial^{+} x_{i}\right)$, by [Had20b, Lemma 1.16] it is in fact covered by two elements of $\Delta^{+} x_{i}$ with opposite orientations. If $w_{i} \in \Delta^{+} x_{i}$ covers $w_{i}$ with orientation + , we have $x_{i} \prec w_{i}^{\prime} \prec w_{i}$.

Dually, we find $w_{i}^{\prime \prime} \in \Delta^{-} x_{i+1}$ that covers $w_{i}$ with orientation -, so that $w_{i} \prec w_{i}^{\prime \prime} \prec x_{i+1}$. It follows that $x_{i} \prec x_{i+1}$ for all $i \in\{0, \ldots, m-1\}$, and we conclude that $x \preceq y$.

Now suppose that $k=0$. A path from $x$ to $y$ in $\mathscr{M}_{0} U$ is a concatenation of two-step paths $x_{i} \rightarrow w_{i} \rightarrow x_{i+1}$ where $\operatorname{dim}\left(x_{i}\right) \in\{1,2\}$ and $w_{i}$ is the only element of $\partial_{0}^{+} x_{i}=\partial_{0}^{-} x_{i+1}$. If $\operatorname{dim}\left(x_{i}\right)=1$ then immediately $x_{i} \prec w_{i}$, otherwise there is exactly one element $w_{i}^{\prime} \in \Delta^{+} x_{i}$ such that $\partial_{0}^{+} x_{i}=\partial^{+} w_{i}^{\prime}$, so $x_{i} \prec w_{i}^{\prime} \prec w_{i}$. Similarly we find that $w_{i} \prec x_{i+1}$.

Corollary 3.16 - If $U$ is a regular 2-molecule, the restriction of $\preceq$ to 2-dimensional elements determines a 1-order on $U$.

3.17 (Normal 1-order). Let $U$ be a regular 2-molecule. The normal 1-order on $U$ is the 1-order determined by Corollary 3.16 .

Example 3.18. In the shape of the 2-diagrams
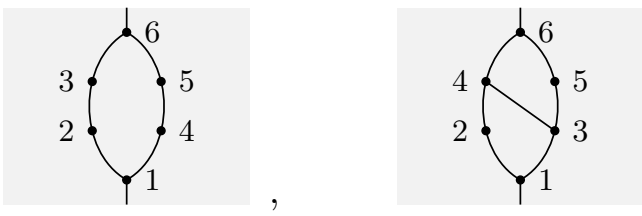

the normal 1-order is indicated by the labels of 2-cells.

In general, a rule-of-thumb for reconstructing the normal 1-order from a string diagram is:

1. if there is an upward path between two 2-cells, then the lowermost precedes the uppermost;

2. if there is no such path, then the leftmost precedes the rightmost.

Due to a certain flexibility in the depiction of string diagrams, the second rule may not always strictly hold (but it will hold up to a harmless deformation of the picture).

Corollary 3.19 - Let $P$ be a regular directed complex with $\operatorname{dim}(P) \leq 2$. Then $P$ is frame-acyclic.

Lemma 3.20 - Let $U$ be a regular molecule with $\operatorname{dim}(U) \leq 2$ and let $I \subseteq U$ be a 1-molecule with $\partial^{-} I=\partial_{0}^{-} U$ and $\partial^{+} I=\partial_{0}^{+} U$. Then

(a) there is a unique decomposition $U=U_{+} \#_{1} U_{-}$with $\partial_{1}^{+} U_{+}=\partial_{1}^{-} U_{-}=I$; 
(b) for all $\alpha \in\{+,-\}$, if $V \sqsubseteq U$ is a 2-molecule with spherical boundary and $V \cap I=\partial^{\alpha} V$, then $V \sqsubseteq U_{\alpha}$.

Proof. By induction on the number $m$ of 2-dimensional elements of $U$ : if $m=0$, then necessarily $U=I$ and $U=I \#_{1} I$ is the unique decomposition. If $m>0$, we can write $U=U^{\prime} \#_{1} U_{x}$ where $U_{x}$ contains a single 2-dimensional element $x$. Now either

- $I \subseteq U^{\prime}$, in which case we have a unique decomposition $U^{\prime}=U_{+}^{\prime} \#_{1} U_{-}^{\prime}$ and we can set $U_{+}:=U_{+}^{\prime}$ and $U_{-}:=U_{-}^{\prime} \#_{1} U_{x}$, or

- $\partial^{+} x \subseteq I$, since $I$ traces a path in $\mathscr{H}^{\circ} U$ through 0-dimensional and 1-dimensional elements, and given that $\partial^{+} x \subseteq \partial^{+} U$, such a path can only enter $\partial^{+} x$ through $\partial_{0}^{-} x$, traverse the entire $\partial^{+} x$, and leave through $\partial_{0}^{+} x$. Then $I^{\prime}:=I\left[\partial^{-} x / \partial^{+} x\right]$ is well-defined and a 1 -molecule in $U^{\prime}$; by the inductive hypothesis, we have a decomposition $U^{\prime}=U_{+}^{\prime} \#_{1} U_{-}^{\prime}$ relative to $I^{\prime}$. Then setting $U_{+}:=U_{+}^{\prime} \cup \operatorname{cl}\{x\}$ and $U_{-}:=U_{-}^{\prime}$ produces a decomposition of $U$ relative to $I$.

Uniqueness is straightforward since the removal of $\{x\} \cup\left(\partial^{+} x \backslash \partial_{0} x\right)$ from a decomposition of $U$ produces a decomposition of $U^{\prime}$ either relative to $I$ or to $I^{\prime}$.

Let $V \sqsubseteq U$ be a 2-molecule with spherical boundary and $V \cap I=\partial^{\alpha} V$. If $V$ is an atom, then clearly $V \sqsubseteq U_{\alpha}$. Otherwise, observe that $I$ is not affected by the substitution $U[\langle V\rangle / V]$ and $\partial^{\alpha}\langle V\rangle=\partial^{\alpha} V \subseteq I$. Decomposing $U[\langle V\rangle / V]$ as $U_{+}^{\prime} \#_{1} U_{-}^{\prime}$, by the atom case we have $\langle V\rangle \sqsubseteq U_{\alpha}^{\prime}$. Now $U_{\alpha}^{\prime}[V /\langle V\rangle]$ and $U_{-\alpha}^{\prime}$ are factors of a decomposition of $U$ relative to $I$, so by uniqueness $U_{\alpha}=U_{\alpha}^{\prime}[V /\langle V\rangle]$ and $V \sqsubseteq U_{\alpha}$.

Proposition 3.21 - Let $U, V, W$ be regular 2-molecules. Suppose $V$ and $W$ have spherical boundary, $V, W \sqsubseteq U$, and $V \cap W \subseteq \partial V \cup \partial W$. Then $W \sqsubseteq U[\langle V\rangle / V]$ and $V \sqsubseteq U[\langle W\rangle / W]$.

Proof. Fix $\alpha \in\{+,-\}$ and let $U^{\prime}:=U[\langle V\rangle / V]$; by assumption, as a closed subset $W$ is unaffected by this substitution.

We construct a sequence of 1-molecules $I_{0}, \ldots, I_{n}$ as follows. Let $I_{0}:=\partial^{\alpha} W$. For $i \geq 0$, if $\partial^{-} I_{i}=\partial_{0}^{-} U$, then let $k:=i$ and move to the next cycle, otherwise pick a 1-dimensional element $x$ with $\partial^{+} x=\partial^{-} I_{i}$ and let $I_{i+1}:=\operatorname{cl}\{x\} \#_{0} I_{i}$.

For $i \geq k$, if $\partial^{+} I_{i}=\partial_{0}^{+} U$, then let $n:=i$ and stop, otherwise pick a 1-dimensional element $x$ with $\partial^{-} x=\partial^{+} I_{i}$ and let $I_{i+1}:=I_{i} \#_{0} \operatorname{cl}\{x\}$. This process terminates by finiteness of $U^{\prime}$ and acyclicity of $\mathscr{H}^{\circ} U$.

Now $I:=I_{n}$ is unaffected by the reverse substitution $U=U^{\prime}[V /\langle V\rangle]$, has $\partial^{-} I=\partial_{0}^{-} U$ and $\partial^{+} I=\partial_{0}^{+} U$, and $W \cap I=\partial^{\alpha} W$. Consider the unique 
decomposition $U_{+}^{\prime} \#_{1} U_{-}^{\prime}$ of $U^{\prime}$ relative to $I$ given by Lemma 3.20. Clearly $\langle V\rangle \sqsubseteq U_{\beta}^{\prime}$ for some $\beta \in\{+,-\}$, so $U_{\beta}:=U_{\beta}^{\prime}[V /\langle V\rangle]$ and $U_{-\beta}:=U_{-\beta}^{\prime}$ produces the unique decomposition of $U$ relative to $I$.

Now observe that if we decompose relative to $I^{\prime}:=I\left[\partial^{-\alpha} W / \partial^{\alpha} W\right]$ instead of $I$, only the 2-dimensional elements of $W$ "switch sides" in the factorisation, so we can vary $\alpha$ without affecting $\beta$. Choosing $\alpha:=-\beta$, we have

$$
V \sqsubseteq U_{\beta}, \quad W \sqsubseteq U_{-\beta}
$$

and the substitution of $\langle V\rangle$ for $V$, or of $\langle W\rangle$ for $W$, only affects one factor.

Comment 3.22. As a consequence of Proposition 3.21, if $V$ and $W$ are submolecules with spherical boundary of a regular 2-molecule $U$ that only overlap on their boundaries, then they can both be substituted in $U$ : if $U\left[W^{\prime} / W\right]$ and $U\left[V^{\prime} / V\right]$ are both defined as 2-molecules, then so are $U\left[W^{\prime} / W\right]\left[V^{\prime} / V\right]$ and $U\left[V^{\prime} / V\right]\left[W^{\prime} / W\right]$, which are in fact equal. This generalises to an arbitrary number $V_{1}, \ldots, V_{n} \sqsubseteq U$ of 2-molecules such that $V_{i} \cap V_{j} \subseteq \partial V_{i} \cup \partial V_{j}$ for all $i, j \in\{1, \ldots, n\}, i \neq j$.

Dimension 2 is, in fact, the largest dimension in which this result holds. The following is an example of a regular 3-molecule for which the analogous statement fails; it is a simplified version of [Ste93, Section 8], itself based on [Pow91, Example 3.11].

The point in our proof that fails to generalise to higher dimensions is the seemingly innocuous fact that $\partial^{+} W$ can always be extended to a 1-molecule $I$ with $\partial I=\partial_{0} U$. In the example below, $\partial^{+} W$ cannot be extended to any 2-molecule in $U[\langle V\rangle / V]$ whose boundary is equal to $\partial_{1} U$.

Example 3.23. Let $U$ be the shape of the 3-diagram

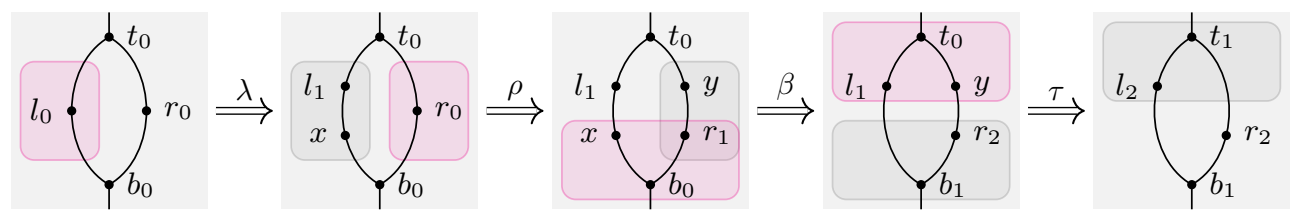

where we use the labels of cells to refer to the corresponding atoms of $U$. Then both $V:=\lambda \cup \tau$ and $W:=\rho \cup \beta$ are submolecules of $U$, they have spherical boundary, and they do not share any 3 -atoms, so they only intersect in the boundary.

However, $W$ is not a submolecule of $U[\langle V\rangle / V]$, and $V$ is not a submolecule of $U[\langle W\rangle / W]$. Indeed, there are paths

$$
\rho \rightarrow y \rightarrow\langle V\rangle \rightarrow x \rightarrow \beta, \quad \lambda \rightarrow x \rightarrow\langle W\rangle \rightarrow y \rightarrow \tau
$$


in $\mathscr{M}_{2} U[\langle V\rangle / V]$ and $\mathscr{M}_{2} U[\langle W\rangle / W]$, respectively; note that we are confusing an atom with its greatest element. If $W \sqsubseteq U[\langle V\rangle / V]$ or $V \sqsubseteq U[\langle W\rangle / W]$, then it would be possible to substitute $\langle W\rangle$ for $W$ in $U[\langle V\rangle / V]$, or $\langle V\rangle$ for $V$ in $U[\langle W\rangle / W]$, to obtain a regular 3-molecule $U^{\prime}$. These paths would then become cycles in $\mathscr{M}_{2} U^{\prime}$, contradicting Proposition 3.8.

Theorem 3.24 - Let $P$ be a regular directed complex with $\operatorname{dim}(P) \leq 3$. Then $P$ is frame-acyclic.

Proof. It suffices to show that for all regular 3-molecules $U$, if $\operatorname{frdim}(U)=k$, then $\mathscr{M}_{k} U$ is acyclic. The case $k=2$ is handled by Proposition 3.8, so suppose $k \in\{0,1\}$.

By [Had20b, Lemma 2.5] we can decompose $U$ as $V_{1} \#_{2} \ldots \#_{2} V_{m}$, where $V_{i}$ contains a unique 3 -dimensional element $x_{i}$ and $\partial^{\alpha} V_{i}=\partial^{-\alpha} V_{i}\left[\partial^{\alpha} x_{i} / \partial^{-\alpha} x_{i}\right]$ for all $\alpha \in\{+,-\}$ and $i \in\{1, \ldots, m\}$.

Since $\operatorname{cl}\left\{x_{i}\right\} \cap \operatorname{cl}\left\{x_{j}\right\}$ has dimension at most 1 when $i \neq j$, we have that

1. $\partial^{-} x_{i} \subseteq \partial^{-} U$ for all $i \in\{1, \ldots, m\}$, and

2. by [Had20a, Lemma 18], $\operatorname{cl}\left\{x_{i}\right\} \cap \operatorname{cl}\left\{x_{j}\right\}=\partial^{-} x_{i} \cap \partial^{-} x_{j} \subseteq \partial_{1} x_{i} \cup \partial_{1} x_{j}$ when $i \neq j$.

Since for all $i \in\{1, \ldots, m\}$ we have $\partial^{-} x_{i} \sqsubseteq \partial^{-} V_{i}$ and

$$
\partial^{-} U=\partial^{-} V_{i}\left[\partial^{-} x_{i-1} / \partial^{+} x_{i-1}\right] \ldots\left[\partial^{-} x_{1} / \partial^{+} x_{1}\right]
$$

applying Proposition 3.21 repeatedly we find that $\partial^{-} x_{i} \sqsubseteq \partial^{-} U$ and the simultaneous substitution

$$
U^{\prime}:=\partial^{-} U\left[\left\langle\partial^{-} x_{1}\right\rangle / \partial^{-} x_{1}\right] \ldots\left[\left\langle\partial^{-} x_{m}\right\rangle / \partial^{-} x_{m}\right]
$$

is defined as a regular 2-molecule with the same frame dimension as $U$.

Now from every path in $\mathscr{M}_{k} U$, we construct a path in $\mathscr{M}_{k} U^{\prime}$ as follows. The path in $\mathscr{M}_{k} U$ is a concatenation of two-step paths $y_{-} \rightarrow x \rightarrow y_{+}$, where $x$ is maximal in $U, y_{-} \in \partial_{1}^{-} x$ and $y_{+} \in \partial_{1}^{+} x$.

If $\operatorname{dim}(x)<3$, then this path stays inside $\partial^{-} U$, and $x, y_{-}, y_{+}$are unaffected by the substitution (6). If $\operatorname{dim}(x)=3$, then this path can be replaced by a path $y_{-} \rightarrow \tilde{x} \rightarrow y_{+}$in $\mathscr{M}_{k} U^{\prime}$, where $\tilde{x}$ is the greatest element of $\left\langle\partial^{-} x\right\rangle$.

Assuming there is a cycle in $\mathscr{M}_{k} U$, with this procedure we construct a cycle in $\mathscr{M}_{k} U^{\prime}$, which contradicts Corollary 3.19. Thus $\mathscr{M}_{k} U$ is acyclic. 


\section{Pros And Diagrammatic Sets}

\subsection{Diagrammatic nerve of a pro}

4.1. Given a regular directed complex $P$ and $n \in \mathbb{N}$, let $\sigma_{\leq n} P \subseteq P$ be the closed subset of elements $x \in P$ with $\operatorname{dim}(x) \leq n$. Then $\mathcal{M o \ell}\left(\sigma_{\leq n} P\right)^{*}$ and $\sigma_{\leq n} \mathcal{M o l} P^{*}$ are isomorphic $n$-categories.

By Lemma 3.5 combined with Theorem 3.24, for $n \leq 3$ the $n$-category $\sigma_{\leq n} \mathcal{M} o \ell P^{*}$ admits the structure of a polygraph with $\{\operatorname{cl}\{x\} \mid \operatorname{dim}(x) \leq n\}$ as generating cells. Because, in general, for an $\omega$-category $X$,

$$
\sigma_{\leq k} X=\sigma_{\leq k}\left(\tau_{\leq n} X\right) \text { when } k<n,
$$

the 2-category $\tau_{\leq 2} \mathcal{M}$ o $P^{*}$ has the structure of a bicoloured pro with generators $\{\operatorname{cl}\{x\} \mid \operatorname{dim}(x) \leq 1\}$.

Moreover, if $f: P \rightarrow Q$ is a morphism in $\mathbf{D C} \mathbf{p p x}^{\mathcal{R}}$, then $\tau_{\leq 2} \mathcal{M} o \ell f^{*}$ sends each generator $\operatorname{cl}\{x\}$ to a generator $\operatorname{cl}\{f(x)\}$, so it is compatible with this structure. This defines a functor $\mathrm{P}: \mathbf{D C p x}{ }^{\mathcal{R}} \rightarrow \mathbf{P r o}_{b i}$ that fits into a commutative square

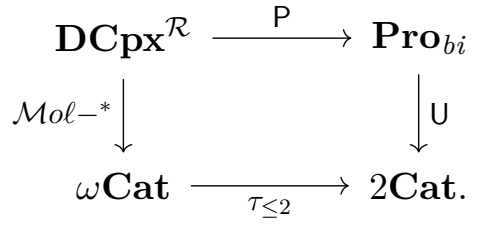

Because $\mathbf{D C p} \mathbf{p x}^{\mathcal{R}}$ is small, $\odot$ Set is locally small, and by Corollary 2.16 Pro $_{b i}$ has all small colimits, by [Rie17, Corollary 6.2.6] the left Kan extension of P: $\mathbf{D C} \mathbf{p x}^{\mathcal{R}} \rightarrow$ Pro $_{b i}$ along the embedding $\mathbf{D C} \mathbf{p} \mathbf{x}^{\mathcal{R}} \hookrightarrow \odot$ Set exists. This produces a functor P: $\odot$ Set $\rightarrow$ Pro $_{b i}$.

Remark 4.2. We may reason as in $[\operatorname{Had} 20 \mathrm{~b}$, Proposition 7.10$]$ to show that $\mathrm{P}: \mathbf{D C} \mathbf{p} \mathbf{x}^{\mathcal{R}} \rightarrow \mathbf{P r o}_{b i}$ preserves the colimits that are already in $\mathbf{D C} \mathbf{p} \mathbf{x}^{\mathcal{R}}$, and deduce from [Corollary 1.34, ibid.] that the left Kan extension of $\mathrm{P}$ along $\mathbf{D C p x}^{\mathcal{R}} \hookrightarrow \odot$ Set is the left Kan extension of its restriction to $\odot$ along the Yoneda embedding.

4.3 (Diagrammatic nerve of bicoloured pros). The diagrammatic nerve of bicoloured pros is the right adjoint

$$
\text { N : Pro } \text { Pri }_{b i} \rightarrow \odot \text { Set }
$$

to the functor P: $\odot$ Set $\rightarrow$ Pro $_{b i}$.

4.4. In each bicoloured pro $(T, \mathscr{T})$, 
- morphisms $\mathrm{P} I_{n} \rightarrow(T, \mathscr{T})$ classify 1-cells in $T$ of the form $a_{1} \#_{0} \ldots \#_{0} a_{n}$, where $a_{i} \in \mathscr{T}$ (including $\mathscr{T}_{0}$ ) for all $i \in\{1, \ldots, n\}$, and

- morphisms $\mathrm{P} U_{n, m} \rightarrow(T, \mathscr{T})$ in Pro $\mathbf{P}_{b i}$ classify 2-cells of type

$$
a_{1} \#_{0} \ldots \#_{0} a_{n} \Rightarrow b_{1} \#_{0} \ldots \#_{0} b_{m}
$$

in $T$ where $a_{i}, b_{j} \in \mathscr{T}$.

These correspond to morphisms $I_{n} \rightarrow \mathrm{N}(T, \mathscr{T})$ and $U_{n, m} \rightarrow \mathrm{N}(T, \mathscr{T})$, respectively, in $\odot$ Set, that is, 1-diagrams and 2-cells in $\mathrm{N}(T, \mathscr{T})$.

If $U$ is a 3-atom, a morphism $e: U \rightarrow \mathrm{N}(T, \mathscr{T})$ restricts, for each $\alpha \in\{+,-\}$, to a 2-diagram $\partial^{\alpha} e$ of shape $\partial^{\alpha} U$ in $\mathrm{N}(T, \mathscr{T})$, whose transpose $\widehat{\partial^{\alpha} e}: \mathrm{P} \partial^{\alpha} U \rightarrow T$ is a diagram of 2-cells in $T$. Because $T$ is a 2-category,

1. the morphism $\widehat{e}: \mathrm{P} U \rightarrow(T, \mathscr{T})$ exhibits an equation between the composites of the diagrams $\widehat{\partial^{+} e}$ and $\widehat{\partial^{-} e}$ in $T$, and

2. if $e^{\prime}: U \rightarrow \mathrm{N}(T, \mathscr{T})$ is another 3-cell with $\partial^{\alpha} e^{\prime}=\partial^{\alpha} e$ for all $\alpha \in\{+,-\}$, then $e=e^{\prime}$.

More in general, if $U$ is an atom, then a cell $U \rightarrow \mathrm{N}(T, \mathscr{T})$ is uniquely determined by its restriction $\sigma_{\leq 2}$ e to $\sigma_{\leq 2} U \subseteq U$.

Lemma 4.5 - Let $X$ be a diagrammatic set, $(T, \mathscr{T})$ a bicoloured pro, and let $f, g: X \rightarrow \mathrm{N}(T, \mathscr{T})$ be morphisms of diagrammatic sets. If $f(x)=g(x)$ for all 2-cells $x$ in $X$, then $f=g$.

Proof. Let $x: U \rightarrow X$ be a cell in $X$ with $\operatorname{dim}(U)>2$. Then $f(x)$ and $g(x)$ are uniquely determined by their restrictions

$$
\sigma_{\leq 2}(f(x))=\left(\sigma_{\leq 2} x\right) ; f, \quad \sigma_{\leq 2}(g(x))=\left(\sigma_{\leq 2} x\right) ; g
$$

to the directed complex $\sigma_{\leq 2} U$. If $f$ and $g$ agree on 2-cells, these are equal.

Proposition 4.6 - The functor $\mathrm{N}$ is full and faithful.

Proof. Suppose $\mathrm{N} f=\mathrm{N} g$ for two morphisms $f, g:(T, \mathscr{T}) \rightarrow(S, \mathscr{S})$ in $\mathbf{P r o}_{b i}$. Given a 2-cell $\varphi:\left(a_{1}, \ldots, a_{n}\right) \Rightarrow\left(b_{1}, \ldots, b_{m}\right)$ in $T$, classified by a morphism $\varphi: \mathrm{P} U_{n, m} \rightarrow(T, \mathscr{T})$ with transpose $\widehat{\varphi}: U_{n, m} \rightarrow \mathrm{N}(T, \mathscr{T})$, we have

$$
\widehat{\varphi} ; \mathrm{N} f=\widehat{\varphi} ; \mathrm{N} g
$$

in $\odot$ Set. It follows that $\varphi ; f=\varphi ; g$ in $\mathbf{P r o}_{b i}$, that is, $f(\varphi)=g(\varphi)$. Because $f$ and $g$ agree on all 2-cells, they are equal. This proves that $\mathrm{N}$ is faithful.

Let $f^{\prime}: \mathrm{N}(T, \mathscr{T}) \rightarrow \mathrm{N}(S, \mathscr{S})$ be a morphism of diagrammatic sets. Given a 2-cell $\varphi:\left(a_{1}, \ldots, a_{n}\right) \Rightarrow\left(b_{1}, \ldots, b_{m}\right)$ in $T$, classified by $\varphi: \mathrm{P} U_{n, m} \rightarrow(T, \mathscr{T})$ 
with transpose $\widehat{\varphi}: U_{n, m} \rightarrow \mathrm{N}(T, \mathscr{T})$, we define $f(\varphi)$ to be the unique 2-cell in $S$ whose classifying morphism $f(\varphi): \mathrm{P} U_{n, m} \rightarrow(S, \mathscr{S})$ is the transpose of $\widehat{\varphi} ; f^{\prime}: U_{n, m} \rightarrow \mathrm{N}(S, \mathscr{S})$.

We want to show that $f$ determines a morphism of bicoloured pros. It is straightforward to verify that $f$ is compatible with all boundaries and with composition and units for 1-cells.

Let $x$ be a 1-cell in $T$, classified by $x: \mathrm{P} I_{n} \rightarrow(T, \mathscr{T})$ with transpose $\widehat{x}$; the unit $\varepsilon x$ is classified by $\varepsilon x: \mathrm{P} U_{n, n} \rightarrow(T, \mathscr{T})$ with transpose $\widehat{\varepsilon x}$. Let

$$
U:=O\left(I_{n}\right) \Rightarrow U_{n, n}
$$

where $O(-)$ is the construction of [Had20b, §2.21]. This is well-defined as a regular 3-atom. There is a unique cell $e: U \rightarrow \mathrm{N}(T, \mathscr{T})$ such that

1. $e$ is equal to $\varepsilon \widehat{x}$ on $\partial^{-} U$, see $[\S 4.16$, ibid.], and

2. $e$ is equal to $\widehat{\varepsilon x}$ on $\partial^{+} U$.

Then $e ; f^{\prime}: U \rightarrow \mathrm{N}(S, \mathscr{S})$ is a 3-cell of type $\varepsilon f^{\prime}(\widehat{x}) \Rightarrow f^{\prime}(\widehat{\varepsilon x})$, whose transpose exhibits the equation $\varepsilon f(x)=f(\varepsilon x)$ in $S$.

Next, let $\varphi, \psi$ be 2-cells in $T$, classified by morphisms

$$
\varphi: \mathrm{P} U_{n, m} \rightarrow(T, \mathscr{T}), \quad \psi: \mathrm{P} U_{p, \ell} \rightarrow(T, \mathscr{T})
$$

with transposes $\widehat{\varphi}, \widehat{\psi}$. Suppose that $\varphi_{\#_{1}} \psi$ is defined; then we may assume $p=m$, and the composite is classified by

$$
\varphi \#_{1} \psi: \mathrm{P} U_{n, \ell} \rightarrow(T, \mathscr{T})
$$

with transpose $\widehat{\varphi \#_{1} \psi}$. Let

$$
U:=\left(U_{n, m} \#_{1} U_{m, \ell}\right) \Rightarrow U_{n, \ell}
$$

this is a regular 3-atom. There is a unique cell $e: U \rightarrow \mathrm{N}(T, \mathscr{T})$ such that

1. $e$ is equal to $\widehat{\varphi}$ on $U_{n, m} \hookrightarrow \partial^{-} U$ and to $\widehat{\psi}$ on $U_{m, \ell} \hookrightarrow \partial^{-} U$, and

2. $e$ is equal to $\widehat{\varphi \#_{1} \psi}$ on $\partial^{+} U$.

Then $e ; f^{\prime}: U \rightarrow \mathrm{N}(S, \mathscr{S})$ is a cell of type

$$
f^{\prime}(\widehat{\varphi}) \#_{1} f^{\prime}(\widehat{\psi}) \Rightarrow f^{\prime}\left(\widehat{\varphi \#_{1} \psi}\right),
$$

whose transpose exhibits an equation $f(\varphi) \#_{1} f(\psi)=f\left(\varphi \#_{1} \psi\right)$ in $S$.

Finally, suppose that $\varphi \#_{0} \psi$ is defined; this composite is classified by

$$
\varphi \#_{0} \psi: \mathrm{P} U_{n+p, m+\ell} \rightarrow(T, \mathscr{T})
$$


with transpose $\widehat{\varphi \#_{0} \psi}$. Let

$$
U:=\left(\left(U_{n, m} \#_{0} U_{p, \ell}\right) \#_{1} U_{m+\ell, m+\ell}\right) \Rightarrow U_{n+p, m+\ell} .
$$

This is a regular 3 -atom and there is a unique cell $e: U \rightarrow \mathrm{N}(T, \mathscr{T})$ such that

1. $e$ is equal to $\widehat{\varphi}$ on $U_{n, m} \hookrightarrow \partial^{-} U$ and to $\widehat{\psi}$ on $U_{p, \ell} \hookrightarrow \partial^{-} U$,

2. $e$ is equal to the transpose of $\varepsilon\left(\partial^{+} \varphi \#_{0} \partial^{+} \psi\right)$ on $U_{m+\ell, m+\ell} \hookrightarrow \partial^{-} U$, and

3. $e$ is equal to $\widehat{\varphi \#_{0} \psi}$ on $\partial^{+} U$.

Then $e ; f^{\prime}: U \rightarrow \mathrm{N}(S, \mathscr{S})$ is a cell of type

$$
\left(f^{\prime}(\widehat{\varphi}) \#_{0} f^{\prime}(\widehat{\psi})\right) \#_{1} f^{\prime}\left(\varepsilon\left(\partial^{+} \widehat{\varphi \#_{0}} \partial^{+} \psi\right)\right) \Rightarrow f^{\prime}\left(\widehat{\varphi \#_{0} \psi}\right) .
$$

whose transpose exhibits the equation

$$
\left(f(\varphi) \#_{0} f(\psi)\right) \#_{1} f\left(\varepsilon\left(\partial^{+} \varphi \#_{0} \partial^{+} \psi\right)\right)=f\left(\varphi \#_{0} \psi\right)
$$

in $S$. Because we already know that $f$ is compatible with units, we deduce that $f(\varphi) \#_{0} f(\psi)=f\left(\varphi \#_{0} \psi\right)$.

This proves that $f:(T, \mathscr{T}) \rightarrow(S, \mathscr{S})$ is a morphism of bicoloured pros. Now $\mathrm{N} f$ and $f^{\prime}$ are morphisms $\mathrm{N}(T, \mathscr{T}) \rightarrow \mathrm{N}(S, \mathscr{S})$ that, by construction, agree on all 2-cells of $\mathrm{N}(T, \mathscr{T})$. It follows from Lemma 4.5 that $\mathrm{N} f=f^{\prime}$. This proves that $\mathrm{N}$ is full.

Comment 4.7. String diagrams are commonly used to depict cells in a pro, usually after an appeal to the Joyal-Street soundness result [JS91]. The diagrammatic nerve construction offers an alternative justification, where diagrams are attributed a combinatorial, rather than topological interpretation.

Unless otherwise stated, our string diagrams will represent diagrams in a diagrammatic set. A caveat is that, contrary to custom, we are not allowed to have nodes with no input or output wires; instead, we need to explicitly introduce units and unitors [Had20b, §4.17] where necessary.

To distinguish them visually, we draw unit 1-cells as dotted wires, and unitor 2-cells as "dotless nodes": for example, a 2-cell of type $(0) \Rightarrow(1)$ in a one-sorted pro will be depicted as

as opposed to

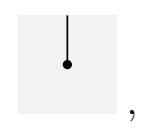

while a left unitor 2-cell will be depicted as

as opposed to

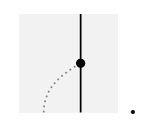

This may seem like unnecessary trouble in dimension 2 ; the pay-off is that diagrammatic sets provide sound diagrammatic reasoning in all dimensions. 


\subsection{Realisation of diagrammatic sets in Gray-categories}

4.8. Our next goal is to construct a functor G: $\mathbf{D C} \mathbf{p p x}^{\mathcal{R}} \rightarrow$ GrayCat, different from the "obvious" one obtained by composing $\mathcal{M} o \ell-{ }^{*}: \mathbf{D C p x}^{\mathcal{R}} \rightarrow \omega \mathbf{C a t}$ with $\tau_{\leq 3}: \omega$ Cat $\rightarrow 3$ Cat and then including 3Cat in GrayCat. In particular, GP will in general have non-trivial interchangers, so it will not be a strict 3-category.

Every regular directed complex is the colimit of the diagram of inclusions of its atoms $[\operatorname{Had} 20 \mathrm{~b}$, Corollary 1.34]. We impose that $\mathrm{G}$ preserve these colimit diagrams. Then it suffices to define $G$ on atoms of increasing dimension. For each $n \in \mathbb{N}+\{-1\}$, let $\odot_{n}$ be the full subcategory of $\odot$ on the atoms of dimension $\leq n$.

4.9 ( $\mathrm{G}$ in dimension $\leq 2$ ). On regular atoms of dimension $\leq 2$, we define $\mathrm{G}$ to be $\mathcal{M} o \ell-: \odot_{2} \rightarrow 3$ Cat followed by the embedding 3Cat $\hookrightarrow$ GrayCat. We extend $\mathrm{G}$ along colimits to all regular directed complexes of dimension $\leq 2$.

4.10. Let $P$ be a 2-dimensional regular directed complex. Then $\mathrm{G}\left(\sigma_{\leq 1} P\right)$ is equal to (the image under the embedding $3 \mathbf{C a t} \hookrightarrow$ GrayCat of) $\mathcal{M} o \ell \sigma_{\leq 1} P^{*}$ and has the structure of a 1-(pre)polygraph with the 1-atoms of $P$ as generators. Now, for all 2-atoms $x \in P$,

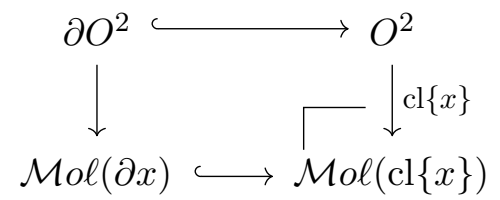

is a pushout both in $\omega$ PreCat and GrayCat. By the dual of the pullback lemma, the pushout of the span

$$
\begin{gathered}
\coprod_{\operatorname{dim}(x)=n} \mathcal{M o \ell}(\partial x) \hookrightarrow \coprod_{\operatorname{dim}(x)=n} \mathcal{M o \ell}(\operatorname{cl}\{x\}), \\
\coprod_{\operatorname{dim}(x)=n} \mathcal{M} o \ell(\partial x) \hookrightarrow \mathcal{M} o \ell \sigma_{\leq 1} P^{*}
\end{gathered}
$$

in $\omega$ PreCat determines a 2-prepolygraph $(\mathrm{GP})_{2}$, while in GrayCat it is equivalent to the construction of GP. The results of [FM18, Section 1.6] imply that

1. $\mathrm{G} P$ is obtained from $(\mathrm{GP})_{2}$ by freely attaching some 3-cells (interchange generators) indexed by generating cells of $(\mathrm{GP})_{2}$, and imposing some equations of 3-cells, so in particular

2. $(\mathrm{GP})_{2}$ is the 2 -skeleton of $\mathrm{GP}$. 
In the terminology of Forest and Mimram, $P$ determines a presentation of the 2-precategory $(\mathrm{GP})_{2}$, which can be completed to a Gray presentation of GP by freely adding the necessary structural generators.

Lemma 4.11 - Let $U$ be a regular 2-molecule. There is a bijective correspondence between

1. cells of rank 2 in $\mathrm{G} U$, and

2. 2-molecules $V \subseteq U$ together with a 1-order.

Proof. By the discussion in $\S 4.10$, the 2-cells in $\mathrm{G} U$ are the same as the 2-cells in the 2-prepolygraph $(\mathrm{GU})_{2}$, so they are freely generated by the atoms of $U$ under principal compositions ( $\$ 1.33)$, subject to the axioms of $\omega$-precategories.

Let $V$ be a 2 -molecule with a 1-order $\left(x_{1}, \ldots, x_{m}\right)$. By Lemma 3.9, we obtain a decomposition

$$
V=V_{1} \#_{1} \ldots \#_{1} V_{m}
$$

Now each $V_{i}$ has frame dimension 0 or -1 , so it has a (clearly unique) decomposition

$$
\operatorname{cl}\left\{y_{i, 1}\right\} \#_{0} \ldots \#_{0} \operatorname{cl}\left\{y_{i, k}\right\} \#_{0} \operatorname{cl}\left\{x_{i}\right\} \#_{0} \operatorname{cl}\left\{y_{i, k+1}\right\} \#_{0} \ldots \#_{0} \operatorname{cl}\left\{y_{i, p}\right\}
$$

where $\operatorname{dim}\left(y_{i, j}\right)=1$ for all $j \in\{1, \ldots, p\}$. Replacing the (8) into (7), we obtain a decomposition of $U$ into atoms using only principal compositions, which determines a cell of rank 2 in $(\mathrm{GU})_{2}$.

Conversely, by [FM18, Proposition 2], every cell $y$ of rank 2 in $(\mathrm{GU})_{2}$ has a unique expression of the form $y_{1} \#_{1} \ldots \#_{1} y_{m}$ where $y_{i}$ is an expression of the form (8). Now the expression of $y$ is also a valid expression for a 2-cell in $\mathcal{M o \ell U}{ }^{*}$, which by Remark 3.14 is equal to $\mathcal{M o \ell U}$, so it determines a 2-molecule $V \subseteq U$ together with a decomposition into atoms. From this decomposition we recover uniquely a 1 -order $\left(x_{1}, \ldots, x_{m}\right)$ on $V$. The two constructions are clearly inverse to each other.

Remark 4.12. By Lemma 4.11, every cell of rank 2 in $\mathrm{G} U$ is identified uniquely by a pair $\left(V,\left(x_{i}\right)_{i=1}^{m}\right)$ of a 2 -molecule and a 1 -order.

More in general, if $P$ is a 2-dimensional regular directed complex, a pair $\left(V,\left(x_{i}\right)_{i=1}^{m}\right)$ of a 2-molecule in $P$ and a 1-order on it determines a unique cell of rank 2 in GP, although these may not exhaust all cells of rank 2 when $P$ is not totally loop-free.

Proposition 4.13 - Let $U$ and $V \subseteq U$ be regular 2-molecules and let $\left(x_{1}, \ldots, x_{m}\right)$ and $\left(x_{1}^{\prime}, \ldots, x_{m}^{\prime}\right)$ be two 1-orders on $V$. Then in $\mathrm{G} U$ there is a unique 3-cell from $\left(V,\left(x_{i}\right)_{i=1}^{m}\right)$ to $\left(V,\left(x_{i}^{\prime}\right)_{i=1}^{m}\right)$. 
Proof. For each 1-order $\left(x_{1}, \ldots, x_{m}\right)$ on $V$, let $\mathrm{w}\left(\left(x_{i}\right)_{i=1}^{m}\right)$ be equal to the number of pairs $(i, j)$ such that $i<j$ but $x_{j} \prec x_{i}$ in the total order on $U$. Then

- $0 \leq \mathrm{w}\left(\left(x_{i}\right)_{i=1}^{m}\right) \leq\left(\begin{array}{c}m \\ 2\end{array}\right)$,

- $\mathrm{w}\left(\left(x_{i}\right)_{i=1}^{m}\right)=0$ if and only if $\left(x_{i}\right)_{i=1}^{m}$ is the normal 1-order, and

- for all non-trivial interchangers $\chi_{x, y}:\left(V,\left(x_{i}\right)_{i=1}^{m}\right) \Rightarrow\left(V,\left(x_{i}^{\prime}\right)_{i=1}^{m}\right)$, we have $\mathrm{w}\left(\left(x_{i}\right)_{i=1}^{m}\right)<\mathrm{w}\left(\left(x_{i}^{\prime}\right)_{i=1}^{m}\right)$.

It follows that $\mathrm{w}(-)$ induces a termination order on the 2-cells of $\mathrm{G} U$ in the terminology of [FM18, Section 2.2]. Because the Gray presentation of GU determined by $U$ as in $\$ 4.10$ has no non-structural 3-generators, it is always locally confluent, so [Theorem 11,ibid.] applies and GU has at most one 3-cell between any parallel pair of 2-cells. This proves uniqueness.

For existence, it suffices to observe that, if $\mathrm{w}\left(\left(x_{i}\right)_{i=1}^{m}\right)>0$, then there is a non-trivial inverse interchanger with input $\left(V,\left(x_{i}\right)_{i=1}^{m}\right)$; we leave the proof as an exercise. Applying inverse interchangers repeatedly, we obtain invertible 3-cells of type

$$
\left(V,\left(x_{i}\right)_{i=1}^{m}\right) \Rightarrow(V \text {, normal 1-order }), \quad\left(V,\left(x_{i}^{\prime}\right)_{i=1}^{m}\right) \Rightarrow(V \text {, normal 1-order }) .
$$

Composing the first with the inverse of the second produces a 3-cell of type $\left(V,\left(x_{i}\right)_{i=1}^{m}\right) \Rightarrow\left(V,\left(x_{i}^{\prime}\right)_{i=1}^{m}\right)$.

4.14 ( $\mathrm{G}$ in dimension 3). Let $U$ be a regular 3-atom. We define $\mathrm{G} U$ to be the pushout

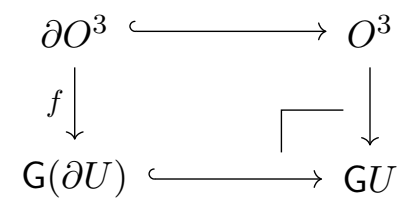

in GrayCat, where $f$ sends $\underline{2}^{\alpha}$ to $\left(\partial^{\alpha} U\right.$, normal 1-order $)$ for each $\alpha \in\{+,-\}$.

Now every map $f: U \rightarrow V$ in $\odot_{3}$ determines an assignment of generators of $\mathrm{G} V$ to generators of $\mathrm{G} U$ which is compatible with boundaries, hence extends uniquely to a functor $\mathrm{G} f: \mathrm{G} U \rightarrow \mathrm{GV}$. This defines $\mathrm{G}: \odot_{3} \rightarrow$ GrayCat. We extend $\mathrm{G}$ along colimits to all regular directed complexes of dimension $\leq 3$.

4.15. By construction, if $P$ is a regular directed complex of dimension 3 , we can associate to each 3-atom $U$ of $P$ a 3 -cell

$$
\llbracket U \rrbracket:\left(\partial^{-} U, \text { normal 1-order }\right) \Rightarrow\left(\partial^{+} U, \text { normal 1-order }\right)
$$

in GP. We want to extend this assignment to all 3-molecules in $P$. 
4.16. Suppose that $U$ contains a single 3 -dimensional element $x$. Then $\partial^{\alpha} x \sqsubseteq \partial^{\alpha} U$ for all $\alpha \in\{+,-\}$, the substitution $\partial^{\alpha} U\left[\left\langle\partial^{\alpha} x\right\rangle / \partial^{\alpha} x\right]$ is welldefined, and

$$
\partial^{-} U\left[\left\langle\partial^{-} x\right\rangle / \partial^{-} x\right]=\partial^{+} U\left[\left\langle\partial^{+} x\right\rangle / \partial^{+} x\right] .
$$

Pick a 1-order $\left(x_{i}\right)_{i=1}^{m}$ on $\partial^{\alpha} U\left[\left\langle\partial^{\alpha} x\right\rangle / \partial^{\alpha} x\right]$; then $\operatorname{cl}\left\{x_{k}\right\}=\left\langle\partial^{\alpha} x\right\rangle$ for a unique $k \in\{1, \ldots, m\}$. Let $y_{1}, \ldots, y_{p}$ be the normal 1-order on $\partial^{-} x$ and let $z_{1}, \ldots, z_{q}$ be the normal 1-order on $\partial^{+} x$. Then

$$
\begin{aligned}
\left(x_{i}^{-}\right)_{i=1}^{m+p-1}: & =\left(x_{1}, \ldots, x_{k-1}, y_{1}, \ldots, y_{p}, x_{k+1}, \ldots, x_{m}\right), \\
\left(x_{i}^{+}\right)_{i=1}^{m+q-1}: & :=\left(x_{1}, \ldots, x_{k-1}, z_{1}, \ldots, z_{q}, x_{k+1}, \ldots, x_{m}\right)
\end{aligned}
$$

are 1-orders on $\partial^{-} U$ and $\partial^{+} U$, respectively.

Now substituting $\llbracket \operatorname{cl}\{x\} \rrbracket$ for $\operatorname{cl}\left\{x_{k}\right\}$ in the decomposition of $\partial^{\alpha} U\left[\left\langle\partial^{\alpha} x\right\rangle / \partial^{\alpha} x\right]$ corresponding to the 1-order $\left(x_{i}\right)_{i=1}^{m}$ yields a valid expression for a 3-cell

$$
c[x]:\left(\partial^{-} U,\left(x_{i}^{-}\right)_{i=1}^{m+p-1}\right) \Rightarrow\left(\partial^{+} U,\left(x_{i}^{+}\right)_{i=1}^{m+q-1}\right)
$$

in GP. By Proposition 4.13, there are unique 3-cells

$$
\begin{aligned}
& \chi^{-}:\left(\partial^{-} U, \text { normal 1-order }\right) \Rightarrow\left(\partial^{-} U,\left(x_{i}^{-}\right)_{i=1}^{m+p-1}\right), \\
& \chi^{+}:\left(\partial^{+} U,\left(x_{i}^{+}\right)_{i=1}^{m+q-1}\right) \Rightarrow\left(\partial^{+} U, \text { normal 1-order }\right)
\end{aligned}
$$

obtained as composites of interchangers and inverse interchangers, respectively. We define $\llbracket U \rrbracket$ to be the composite

$$
\chi^{-} \#_{2} c[x] \#_{2} \chi^{+}:\left(\partial^{-} U \text {, normal 1-order }\right) \Rightarrow\left(\partial^{+} U \text {, normal 1-order }\right) .
$$

We need to show that this is independent of our choice of 1-order $\left(x_{i}\right)_{i=1}^{m}$. Suppose $\left(x_{i}^{\prime}\right)_{i=1}^{m}$ is another 1-order on $\partial^{\alpha} U\left[\left\langle\partial^{\alpha} x\right\rangle / \partial^{\alpha} x\right]$, leading to a potentially different interpretation $\chi^{\prime-} \#_{2} c^{\prime}[x] \#_{2} \chi^{\prime+}$. There are unique 3-cells

$$
\begin{aligned}
& \psi^{-}:\left(\partial^{-} U,\left(x_{i}^{-}\right)_{i=1}^{m+p-1}\right) \Rightarrow\left(\partial^{-} U,\left(x_{i}^{\prime-}\right)_{i=1}^{m+p-1}\right), \\
& \psi^{+}:\left(\partial^{+} U,\left(x_{i}^{+}\right)_{i=1}^{m+q-1}\right) \Rightarrow\left(\partial^{+} U,\left(x_{i}^{\prime+}\right)_{i=1}^{m+q-1}\right)
\end{aligned}
$$

obtained as composites of interchangers and inverse interchangers, and since they "fix" $\partial^{-} x$ and $\partial^{+} x$, by naturality of interchangers we have

$$
\psi^{-} \#_{2} c^{\prime}[x]=c[x] \#_{2} \psi^{+}
$$

hence $c^{\prime}[x]=\left(\psi^{-}\right)^{-1} \#_{2} c[x] \#_{2} \psi^{+}$and

$$
\chi^{\prime-} \#_{2} c^{\prime}[x] \#_{2} \chi^{\prime+}=\chi^{\prime-} \#_{2}\left(\psi^{-}\right)^{-1} \#_{2} c[x] \#_{2} \psi^{+} \#_{2} \chi^{\prime+} .
$$

Finally, by Proposition 4.13, $\chi^{\prime-} \#_{2}\left(\psi^{-}\right)^{-1}=\chi^{-}$and $\psi^{+} \#_{2} \chi^{\prime+}=\chi^{+}$. 
4.17. Let $U$ be any regular 3 -molecule in $P$ and fix a 2 -order $\left(x_{1}, \ldots, x_{m}\right)$ on $U$. Then Lemma 3.9 combined with Theorem 3.24 gives a decomposition $U=V_{1} \#_{2} \ldots \#_{2} V_{m}$ where $x_{i}$ is the only 3-dimensional element of $V_{i}$ for each $i \in\{1, \ldots, m\}$. We let $\llbracket U \rrbracket$ in $G P$ be the composite $\llbracket V_{1} \rrbracket \#_{2} \ldots \#_{2} \llbracket V_{m} \rrbracket$ of the 3 -cells

$$
\llbracket V_{i} \rrbracket:\left(\partial^{-} V_{i}, \text { normal 1-order }\right) \Rightarrow\left(\partial^{+} V_{i}, \text { normal 1-order }\right)
$$

defined in $\$ 4.16$.

We need to show that this interpretation is independent of the 2-order chosen on $U$. Observe that any pair of 2 -orders on $U$ is related by a sequence of elementary transpositions of consecutive elements that are not connected by a path in $\mathscr{M}_{2} U$. Thus it suffices to show that if

$$
W_{1} \#_{2} W_{2}=W_{1}^{\prime} \#_{2} W_{2}^{\prime}
$$

as 3 -molecules, where $x$ is the only 3 -dimensional element in $W_{1}$ and $W_{2}^{\prime}$, while $y$ is the only 3 -dimensional element in $W_{2}$ and $W_{1}^{\prime}$, then

$$
\llbracket W_{1} \rrbracket \#_{2} \llbracket W_{2} \rrbracket=\llbracket W_{1}^{\prime} \rrbracket \#_{2} \llbracket W_{2}^{\prime} \rrbracket .
$$

The interpretation of $W_{1}$ involves a choice of 1-order on $\partial^{\alpha} W_{1}\left[\left\langle\partial^{\alpha} x\right\rangle / \partial^{\alpha} x\right]$ but it is independent of this choice. Now $\partial^{-} y \sqsubseteq \partial^{-} W_{2}=\partial^{+} W_{1}$, and since $x$ and $y$ are not connected by a path in $\mathscr{M}_{2} U$, necessarily

$$
\partial^{+} x \cap \partial^{-} y \subseteq \operatorname{cl}\{x\} \cap \operatorname{cl}\{y\} \subseteq \partial_{1} x \cup \partial_{1} y,
$$

and by Proposition $3.21 \partial^{-} y \sqsubseteq \partial^{+} W_{1}\left[\left\langle\partial^{+} x\right\rangle / \partial^{+} x\right]$.

Applying the known equalities between the boundaries of $W_{1}, W_{2}, W_{1}^{\prime}, W_{2}^{\prime}$, we deduce that the double substitutions

$$
\begin{aligned}
& \partial^{+} W_{1}\left[\left\langle\partial^{+} x\right\rangle / \partial^{+} x\right]\left[\left\langle\partial^{-} y\right\rangle / \partial^{-} y\right], \\
& \partial^{-} W_{2}\left[\left\langle\partial^{-} y\right\rangle / \partial^{-} y\right]\left[\left\langle\partial^{+} x\right\rangle / \partial^{+} x\right], \\
& \partial^{+} W_{1}^{\prime}\left[\left\langle\partial^{+} y\right\rangle / \partial^{+} y\right]\left[\left\langle\partial^{-} x\right\rangle / \partial^{-} x\right], \\
& \partial^{-} W_{2}^{\prime}\left[\left\langle\partial^{-} x\right\rangle / \partial^{-} x\right]\left[\left\langle\partial^{+} y\right\rangle / \partial^{+} y\right]
\end{aligned}
$$

are all well-defined and equal to the same regular 2-molecule. Fix a 1-order $\left(z_{i}\right)_{i=1}^{p}$ on it. Then $\left\langle\partial^{\alpha} x\right\rangle=\operatorname{cl}\left\{z_{k}\right\}$ and $\left\langle\partial^{\beta} y\right\rangle=\operatorname{cl}\left\{z_{\ell}\right\}$ for a unique pair $k, \ell \in\{1, \ldots, p\}$. Now

- to interpret $W_{1}$, choose the 1 -order on $\partial^{+} W_{1}\left[\left\langle\partial^{+} x\right\rangle / \partial^{+} x\right]$ obtained by replacing $z_{\ell}$ with the normal 1-order on $\partial^{-} y$ in $\left(z_{i}\right)_{i=1}^{p}$,

- to interpret $W_{2}$, choose the 1-order on $\partial^{-} W_{2}\left[\left\langle\partial^{-} y\right\rangle / \partial^{-} y\right]$ obtained by replacing $z_{k}$ with the normal 1-order on $\partial^{+} x$ in $\left(z_{i}\right)_{i=1}^{p}$, 
and similarly for $W_{1}^{\prime}$ and $W_{2}^{\prime}$. With the construction of $\S 4.16$, any choices of 1-orders lead to expressions

$$
\begin{aligned}
& \chi_{1}^{-} \#_{2} c[x] \#_{2} \chi_{1}^{+} \#_{2} \chi_{2}^{-} \#_{2} c[y] \#_{2} \chi_{2}^{+}, \\
& \chi_{1}^{\prime-} \#_{2} c^{\prime}[y] \#_{2} \chi_{1}^{\prime+} \#_{2} \chi_{2}^{\prime-} \#_{2} c^{\prime}[x] \#_{2} \chi_{2}^{\prime+},
\end{aligned}
$$

and with the particular choice that we made,

$$
\chi_{1}^{-}=\chi_{1}^{\prime-}, \quad \chi_{2}^{+}=\chi_{2}^{\prime+},
$$

while $\chi_{1}^{+} \#_{2} \chi_{2}^{-}$and $\chi_{1}^{\prime+} \#_{2} \chi_{2}^{\prime-}$ are units, so they can be eliminated. Finally,

$$
\chi_{1}^{-} \#_{2} c[x] \#_{2} c[y] \#_{2} \chi_{2}^{+}=\chi_{1}^{-} \#_{2} c^{\prime}[y] \#_{2} c^{\prime}[x] \#_{2} \chi_{2}^{+}
$$

is a consequence of axiom 4 of Gray-categories. This proves that $\llbracket W_{1} \rrbracket \#_{2} \llbracket W_{2} \rrbracket$ is equal to $\llbracket W_{1}^{\prime} \rrbracket \#_{2} \llbracket W_{2}^{\prime} \rrbracket$, and we conclude that $\llbracket U \rrbracket$ is independent of the choice of 2-order.

Example 4.18. Let $U$ be the shape of diagram (3). We introduce names for some atoms of $U$ as follows:

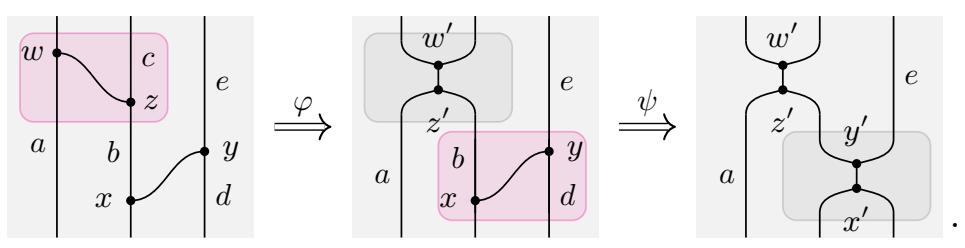

The 3-atoms $\varphi$ and $\psi$ are interpreted in GU as 3-cells

$$
\begin{aligned}
& \llbracket \varphi \rrbracket:\left(a \#_{0} z\right) \#_{1}\left(w \#_{0} c\right) \Rightarrow z^{\prime} \#_{1} w^{\prime} \\
& \llbracket \psi \rrbracket:\left(x \#_{0} d\right) \#_{1}\left(b \#_{0} y\right) \Rightarrow x^{\prime} \#_{1} y^{\prime}
\end{aligned}
$$

notice that in this case both $\partial^{\alpha} \varphi$ and $\partial^{\alpha} \psi$ admit a single 1-order, which is necessarily the normal 1 -order.

We pick the 2-order $(\varphi, \psi)$ on $U$, which determines the decomposition

$$
U=V_{1} \#_{2} V_{2}, \quad V_{1}:=\varphi \cup \partial^{-} \psi, \quad V_{2}:=\psi \cup \partial^{+} \varphi .
$$

To interpret $V_{1}$ in $G U$, first we need to consider $V_{1}\left[\left\langle\partial^{-} \varphi\right\rangle / \partial^{-} \varphi\right]$. This is the shape of the diagram

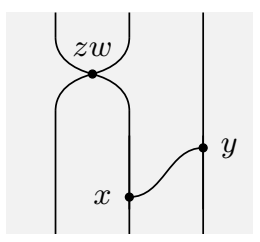


on which we pick the 1 -order $(x, y, z w)$. The 3 -cell $c[\varphi]$ corresponding to this 1-order, defined as in (9), is

$$
\left(a \#_{0} x \#_{0} d\right) \#_{1}\left(a \#_{0} b \#_{0} y\right) \#_{1}\left(\llbracket \varphi \rrbracket \#_{0} e\right)
$$

of type $\left(\partial^{-} V_{1},(x, y, z, w)\right) \Rightarrow\left(\partial^{+} V_{1},\left(x, y, z^{\prime}, w^{\prime}\right)\right)$ in $\mathrm{G} U$.

The normal 1-order on $\partial^{-} V_{1}$ is in fact $(x, z, w, y)$. Applying a pair of interchangers to first move $w$ after $y$, then $z$ after $y$, we obtain a 3-cell

$$
\chi_{1}^{-}:\left(\partial^{-} V_{1},(x, z, w, y)\right) \Rightarrow\left(\partial^{-} V_{1},(x, y, z, w)\right) .
$$

Similarly, the normal 1 -order on $\partial^{+} V_{1}$ is $\left(x, z^{\prime}, w^{\prime}, y\right)$, so we apply a pair of inverse interchangers to move $y$ after $z^{\prime}$, then $y$ after $w^{\prime}$, producing a 3-cell

$$
\chi_{1}^{+}:\left(\partial^{+} V_{1},\left(x, y, z^{\prime}, w^{\prime}\right)\right) \Rightarrow\left(\partial^{+} V_{1},\left(x, z^{\prime}, w^{\prime}, y\right)\right) .
$$

Then $\llbracket V_{1} \rrbracket$ is defined to be $\chi_{1}^{-} \#_{2} c[\varphi] \#_{2} \chi_{1}^{+}$.

Next, consider $V_{2}\left[\left\langle\partial^{-} \psi\right\rangle / \partial^{-} \psi\right]$. This is the shape of the diagram

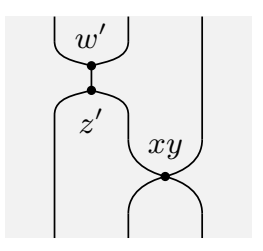

which admits only the 1 -order $\left(x y, z^{\prime}, w^{\prime}\right)$. Correspondingly, we construct the 3-cell

$$
c[\psi]:=\left(a \#_{0} \llbracket \psi \rrbracket\right) \#_{1}\left(z^{\prime} \#_{0} e\right) \#_{1}\left(w^{\prime} \#_{0} e\right)
$$

which is of type $\left(\partial^{-} V_{2},\left(x, y, z^{\prime}, w^{\prime}\right)\right) \Rightarrow\left(\partial^{+} V_{2},\left(x^{\prime}, y^{\prime}, z^{\prime}, w^{\prime}\right)\right)$. The normal 1-order on $\partial^{-} V_{2}$ is $\left(x, z^{\prime}, w^{\prime}, y\right)$, and we have a composite of interchangers

$$
\chi_{2}^{-}:\left(\partial^{-} V_{2},\left(x, z^{\prime}, w^{\prime}, y\right)\right) \Rightarrow\left(\partial^{-} V_{2},\left(x, y, z^{\prime}, w^{\prime}\right)\right),
$$

which is in fact the inverse of $\chi_{1}^{+}$. On the other hand, $\left(x^{\prime}, y^{\prime}, z^{\prime}, w^{\prime}\right)$ is already the normal 1-order on $\partial^{+} V_{2}$, so $\llbracket V_{2} \rrbracket$ is just $\chi_{2}^{-} \#_{2} c[\psi]$. Overall, $\llbracket U \rrbracket$ is

$$
\chi_{1}^{-} \#_{2} c[\varphi] \#_{2} c[\psi]:\left(\partial^{-} U,(x, z, w, y)\right) \Rightarrow\left(\partial^{+} U,\left(x^{\prime}, y^{\prime}, z^{\prime}, w^{\prime}\right)\right) .
$$

If we had picked the 2-order $(\psi, \varphi)$, we would have instead ended up with the expression $\chi_{1}^{-} \#_{2} c^{\prime}[\psi] \#_{2} c^{\prime}[\varphi]$ where

$$
\begin{aligned}
c^{\prime}[\psi] & :=\left(a \#_{0} \llbracket \psi \rrbracket\right) \#_{1}\left(a \#_{0} z \#_{0} e\right) \#_{1}\left(w \#_{0} c \#_{0} e\right), \\
c^{\prime}[\varphi] & :=\left(a \#_{0} x^{\prime}\right) \#_{1}\left(a \#_{0} y^{\prime}\right) \#_{1}\left(\llbracket \varphi \rrbracket \#_{0} e\right) .
\end{aligned}
$$

It follows from axiom 4 of Gray-categories that $c[\varphi] \#_{2} c[\psi]=c^{\prime}[\psi] \#_{2} c^{\prime}[\varphi]$, confirming that $\llbracket U \rrbracket$ is independent of the 2-order on $U$. 
4.19 ( $\mathrm{G}$ in dimension $\geq 4$ ). Let $U$ be a regular 4 -atom. We define $\mathrm{G} U$ to be the quotient of $\mathrm{G}(\partial U)$ by the equation

$$
\llbracket \partial^{-} U \rrbracket=\llbracket \partial^{+} U \rrbracket,
$$

where the 3 -molecules $\partial^{\alpha} U$ are interpreted in $\mathrm{G}(\partial U)$ as by $\S 4.17$.

If $f: U \rightarrow V$ is a map in $\odot_{4}$, its restriction to $\partial U$ determines a functor $\mathrm{G}(\partial f): \mathrm{G}(\partial U) \rightarrow \mathrm{G} V$. If $U$ is a 4-atom, then either $\operatorname{dim}(f(U))<4$ and $f\left(\partial^{-} U\right)=f\left(\partial^{+} U\right)$, or $\operatorname{dim}(f(U))=4, f(U)=V$ and $f\left(\partial^{\alpha} U\right)=\partial^{\alpha} V$ for each $\alpha \in\{+,-\}$. In either case, $\mathrm{G}(\partial f)$ is compatible with the equation $\llbracket \partial^{-} U \rrbracket=\llbracket \partial^{+} U \rrbracket$, so it factors uniquely through a functor $\mathrm{G} f: \mathrm{G} U \rightarrow \mathrm{G} V$.

This defines G: $\odot_{4} \rightarrow$ GrayCat, and we extend it along colimits to all regular directed complexes of dimension $\leq 4$.

Finally, if $f: P \rightarrow Q$ is a map of regular directed complexes of arbitrary dimension, it restricts to a map $\sigma_{\leq 4} f: \sigma_{\leq 4} P \rightarrow \sigma_{\leq 4} Q$, and we let $\mathrm{G} f$ be equal to $\mathrm{G}\left(\sigma_{\leq 4} f\right)$. This defines $\mathrm{G}: \mathbf{D C} \mathbf{p p x}^{\mathcal{R}} \rightarrow$ GrayCat.

Comment 4.20. By construction, $G$ ignores any elements of dimension $>4$. The idea is that, while 4-atoms can contribute non-trivial equations of 3-cells in a Gray-category, higher-dimensional atoms can only contribute trivial "equations of equations" with no visible effect.

4.21. Because GrayCat has all small colimits, we are in the conditions of [Rie17, Corollary 6.2.6] and we can define a functor

$$
\text { G : } \odot \text { Set } \rightarrow \text { GrayCat }
$$

as the left Kan extension of G: DCpx ${ }^{\mathcal{R}} \rightarrow$ GrayCat along the embedding $\operatorname{DCpx}^{\mathcal{R}} \hookrightarrow \odot$ Set.

Remark 4.22. Since we made sure at every step that $\mathrm{G}$ preserve the colimits in $\mathbf{D C p x} \mathbf{x}^{\mathcal{R}}$, this is in fact equal to the left Kan extension of the restriction of $\mathrm{G}$ to $\odot$ along the Yoneda embedding.

Remark 4.23. For the usual reasons, $\mathrm{G}$ has a right adjoint, of which we will not make use. Unlike the diagrammatic nerve of pros, it is not full; see $[\operatorname{Had} 20 \mathrm{~b}$, Remark 7.20] for a counterexample that also applies to the present case.

Comment 4.24. The following (generally non-commutative) diagram of func- 
tors recaps the adjunctions that we have established:

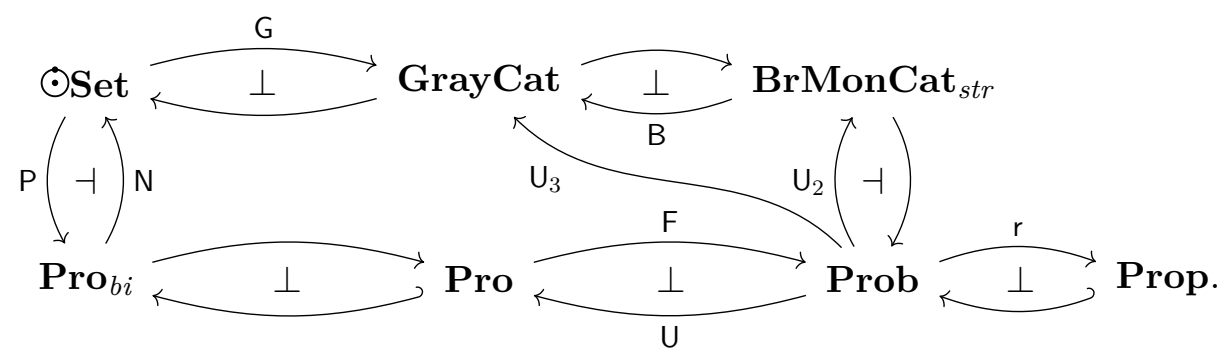

\section{THE SMASH PRODUCT}

\subsection{The tensor product of pros}

We reconstruct the tensor product of props, as defined by Hackney and Robertson, as a reflection of an "external" tensor product of pros producing a prob, whose combinatorics are only slightly more involved.

Lemma 5.1 - Let $s$ be a permutation on the set $\{1, \ldots, n\}$. Then $s$ is either the identity or admits a unique decomposition

$$
s=s_{1} ; \ldots ; s_{p}
$$

with the following properties. For each $i \in\{1, \ldots, p\}$, let $s^{(i)}:=s_{i} ; \ldots ; s_{p}$. Then

1. $s_{i}$ is an elementary transposition $(k k+1)$ of two consecutive elements, and

2. $k$ is the least element of $\{1, \ldots, n\}$ such that $s^{(i)}(k+1)<s^{(i)}(k)$.

Proof. We construct, step by step, decompositions $s=s_{1} ; \ldots ; s_{i-1} ; s^{(i)}$. For $i=1$, we let $s=s^{(1)}$ trivially. For each $i \geq 1$, if $s^{(i)}$ is the identity, we let $p:=i-1$ and we stop.

Otherwise, there exists a least $k$ such that $s^{(i)}(k+1)<s^{(i)}(k)$. We let $s_{i}:=(k k+1)$ and $s^{(i+1)}:=s_{i}^{-1} ; s^{(i)}$. Then $s=s_{1} ; \ldots ; s_{i} ; s^{(i+1)}$.

At each step, the number of pairs $j, j^{\prime} \in\{1, \ldots, n\}$ such that $j<j^{\prime}$ but $s^{(i)}\left(j^{\prime}\right)<s^{(i)}(j)$ strictly decreases, and it is equal to 0 if and only if $s^{(i)}$ is the identity. It follows that the algorithm terminates after a finite number of steps, producing a decomposition with the desired properties.

Uniqueness is clear, since the conditions determine the factor $s_{i}$ uniquely at each step. 
5.2. Let $s$ be a permutation on the set $\{1, \ldots, n\}$. For all 1-cells $\left(a_{1}, \ldots, a_{n}\right)$ in a prob $(T, \mathscr{T})$, we define an invertible 2 -cell

$$
\sigma(s):\left(a_{1}, \ldots, a_{n}\right) \Rightarrow\left(a_{s(1)}, \ldots, a_{s(n)}\right)
$$

in $T$; the dependence of $\sigma(s)$ on $\left(a_{1}, \ldots, a_{n}\right)$ is left implicit.

- If $s$ is the identity, we let $\sigma(s)$ be the unit on $\left(a_{1}, \ldots, a_{n}\right)$.

- If $s$ is an elementary transposition $(k k+1)$ of two consecutive elements, we let

$$
\sigma(s):=a_{1} \#_{0} \ldots \#_{0} a_{k-1 \#_{0}} \sigma_{a_{k}, a_{k+1} \#_{0}} a_{k+2} \ldots \#_{0} a_{n} .
$$

- In general, if $s=s_{1} ; \ldots ; s_{p}$ is the decomposition of $s$ given by Lemma 5.1, we let

$$
\sigma(s):=\sigma\left(s_{1}\right) \#_{1} \ldots \#_{1} \sigma\left(s_{p}\right) .
$$

We also define a second invertible 2-cell

$$
\sigma^{*}(s):\left(a_{1}, \ldots, a_{n}\right) \Rightarrow\left(a_{s(1)}, \ldots, a_{s(n)}\right)
$$

by $\sigma^{*}(s):=\left(\sigma\left(s^{-1}\right)\right)^{-1}$.

Remark 5.3. If $(T, \mathscr{T})$ is a prop, then $\sigma(s)=\sigma^{*}(s)$ for all permutations $s$.

Example 5.4. Let $s$ be the permutation $(1,2,3,4,5) \mapsto(3,1,5,4,2)$. The decomposition of $s$ given by Lemma 5.1 is

$$
s=(23) ;(12) ;(34) ;(45) ;(34) .
$$

We use the graphical notation
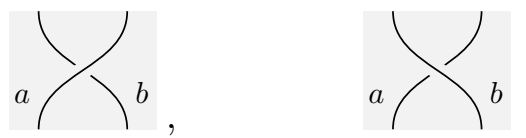

for the braiding $\sigma_{a, b}$ and the inverse braiding $\sigma_{a, b}^{*}$, respectively, in a prob. The 2-cells $\sigma(s)$ and $\sigma^{*}(s)$ of type $\left(a_{1}, a_{2}, a_{3}, a_{4}, a_{5}\right) \Rightarrow\left(a_{3}, a_{1}, a_{5}, a_{4}, a_{2}\right)$ can be pictured as
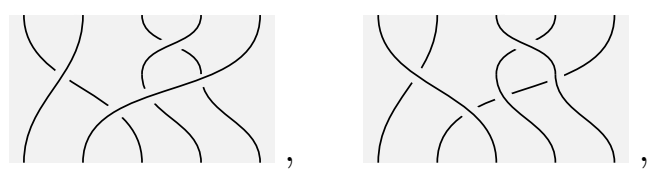

respectively. In a prop, these are identical and may both be pictured as their "shadow"

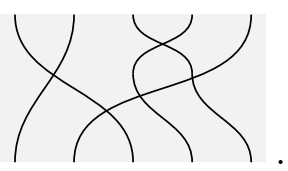


5.5. Let $(T, \mathscr{T})$ be a prob and let $\left\{a_{i, j} \mid 1 \leq i \leq n, 1 \leq j \leq m\right\}$ be a doubly indexed collection of 1-cells in $\mathscr{T}$. We denote by $\left(\left(a_{i, j}\right)_{i=1}^{n}\right)_{j=1}^{m}$ the 1-cell

$$
\left(a_{1,1}, \ldots, a_{n, 1}, a_{1,2}, \ldots, a_{n, 2}, \ldots \ldots, a_{1, m}, \ldots, a_{n, m}\right)
$$

and by $\left(\left(a_{i, j}\right)_{j=1}^{m}\right)_{i=1}^{n}$ the 1-cell

$$
\left(a_{1,1}, \ldots, a_{1, m}, a_{2,1}, \ldots, a_{2, m}, \ldots \ldots, a_{n, 1}, \ldots, a_{n, m}\right) .
$$

We let

$$
\begin{gathered}
\sigma:\left(\left(a_{i, j}\right)_{j=1}^{m}\right)_{i=1}^{n} \Rightarrow\left(\left(a_{i, j}\right)_{i=1}^{n}\right)_{j=1}^{m}, \\
\sigma^{*}:\left(\left(a_{i, j}\right)_{i=1}^{n}\right)_{j=1}^{m} \Rightarrow\left(\left(a_{i, j}\right)_{j=1}^{m}\right)_{i=1}^{n}
\end{gathered}
$$

be equal to $\sigma\left(s^{-1}\right)$ and its inverse $\sigma^{*}(s)$, respectively, for the permutation $s$ implied by the reordering of (10) into (11).

Example 5.6. The 2-cells

$$
\begin{aligned}
\sigma:\left(a_{1,1}, a_{1,2}, a_{1,3}, a_{2,1}, a_{2,2}, a_{2,3}\right) & \Rightarrow\left(a_{1,1}, a_{2,1}, a_{1,2}, a_{2,2}, a_{1,3}, a_{2,3}\right), \\
\sigma^{*}:\left(a_{1,1}, a_{2,1}, a_{1,2}, a_{2,2}, a_{1,3}, a_{2,3}\right) & \Rightarrow\left(a_{1,1}, a_{1,2}, a_{1,3}, a_{2,1}, a_{2,2}, a_{2,3}\right)
\end{aligned}
$$

can be pictured as
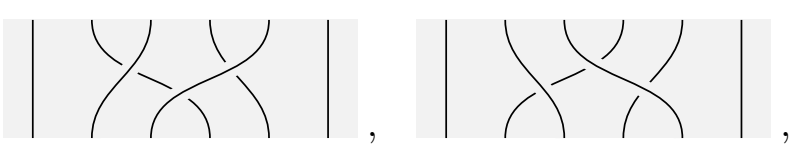

respectively.

5.7 (Tensor product of pros). The tensor product $(T, \mathscr{T}) \otimes(S, \mathscr{S})$ of two pros $(T, \mathscr{T})$ and $(S, \mathscr{S})$ is the prob $(T \otimes S, \mathscr{T} \otimes \mathscr{S})$ constructed as follows.

1. Let $(\mathscr{T} \otimes \mathscr{S})_{0}:=\{\bullet\}$ and $(\mathscr{T} \otimes \mathscr{S})_{1}:=\left\{a \otimes c: \bullet \Rightarrow \bullet \mid a \in \mathscr{T}_{1}, c \in \mathscr{S}_{1}\right\}$. This determines $\sigma_{\leq 1}(T \otimes S)$ together with its 1-polygraph structure, which makes it a pro.

2. Construct the coproducts

$$
\coprod_{c \in \mathscr{S}_{1}}(T, \mathscr{T}), \quad \coprod_{a \in \mathscr{T}_{1}}(S, \mathscr{S})
$$

in Pro. Denote by

$$
-\otimes d:(T, \mathscr{T}) \hookrightarrow \coprod_{c \in \mathscr{S}_{1}}(T, \mathscr{T}), \quad b \otimes-:(S, \mathscr{S}) \hookrightarrow \coprod_{a \in \mathscr{T}_{1}}(S, \mathscr{S})
$$


the inclusions into the $d$-indexed and $b$-indexed summand, respectively. There are morphisms

$$
\sigma_{\leq 1}(T \otimes S) \rightarrow \coprod_{c \in \mathscr{S}_{1}}(T, \mathscr{T}), \quad \sigma_{\leq 1}(T \otimes S) \rightarrow \coprod_{a \in \mathscr{T}_{1}}(S, \mathscr{S})
$$

uniquely determined by the "tautologous" assignments $a \otimes c \mapsto a \otimes c$. Construct the pushout

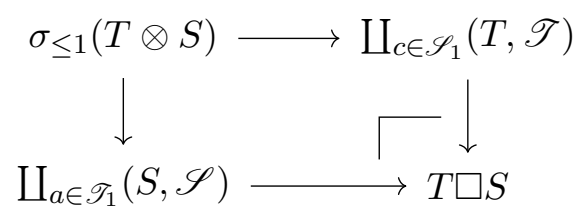

in Pro.

3. Construct the free prob $\mathrm{F}(T \square S)$ and quotient it by the following equations: for all 2-cells

$$
\varphi:\left(a_{1}, \ldots, a_{n}\right) \Rightarrow\left(b_{1}, \ldots, b_{m}\right) \text { in } T, \psi:\left(c_{1}, \ldots, c_{p}\right) \Rightarrow\left(d_{1}, \ldots, d_{q}\right) \text { in } S,
$$

the 1-composite of

$$
\begin{aligned}
&\left(a_{1} \otimes \psi\right) \#_{0} \ldots \#_{0}\left(a_{n} \otimes \psi\right):\left(\left(a_{i} \otimes c_{k}\right)_{k=1}^{p}\right)_{i=1}^{n} \Rightarrow\left(\left(a_{i} \otimes d_{\ell}\right)_{\ell=1}^{q}\right)_{i=1}^{n}, \\
& \sigma:\left(\left(a_{i} \otimes d_{\ell}\right)_{\ell=1}^{q}\right)_{i=1}^{n} \Rightarrow\left(\left(a_{i} \otimes d_{\ell}\right)_{i=1}^{n}\right)_{\ell=1}^{q}, \\
&\left(\varphi \otimes d_{1}\right) \#_{0} \ldots \#_{0}\left(\varphi \otimes d_{q}\right):\left(\left(a_{i} \otimes d_{\ell}\right)_{i=1}^{n}\right)_{\ell=1}^{q} \Rightarrow\left(\left(b_{j} \otimes d_{\ell}\right)_{j=1}^{m}\right)_{\ell=1}^{q}, \\
& \sigma^{*}:\left(\left(b_{j} \otimes d_{\ell}\right)_{j=1}^{m}\right)_{\ell=1}^{q} \Rightarrow\left(\left(b_{j} \otimes d_{\ell}\right)_{\ell=1}^{q}\right)_{j=1}^{m}
\end{aligned}
$$

is equal to the 1-composite of

$$
\begin{aligned}
& \sigma:\left(\left(a_{i} \otimes c_{k}\right)_{k=1}^{p}\right)_{i=1}^{n} \Rightarrow\left(\left(a_{i} \otimes c_{k}\right)_{i=1}^{n}\right)_{k=1}^{p}, \\
&\left(\varphi \otimes c_{1}\right) \#_{0} \ldots \#_{0}\left(\varphi \otimes c_{p}\right):\left(\left(a_{i} \otimes c_{k}\right)_{i=1}^{n}\right)_{k=1}^{p} \Rightarrow\left(\left(b_{j} \otimes c_{k}\right)_{j=1}^{m}\right)_{k=1}^{p}, \\
& \sigma^{*}:\left(\left(b_{j} \otimes c_{k}\right)_{j=1}^{m}\right)_{k=1}^{p} \Rightarrow\left(\left(b_{j} \otimes c_{k}\right)_{k=1}^{p}\right)_{j=1}^{m}, \\
&\left(b_{1} \otimes \psi\right) \#_{0} \ldots \#_{0}\left(b_{m} \otimes \psi\right):\left(\left(b_{j} \otimes c_{k}\right)_{k=1}^{p}\right)_{j=1}^{m} \Rightarrow\left(\left(b_{j} \otimes d_{\ell}\right)_{\ell=1}^{q}\right)_{j=1}^{m} .
\end{aligned}
$$

We label this equation $\varphi \otimes \psi$.

Note that any composite indexed by an empty list must be interpreted as a unit on $\bullet$ of the appropriate dimension.

If $f:(T, \mathscr{T}) \rightarrow\left(T^{\prime}, \mathscr{T}^{\prime}\right)$ and $g:(S, \mathscr{S}) \rightarrow\left(S^{\prime}, \mathscr{S}^{\prime}\right)$ are morphisms of pros, we can define morphisms

$$
\begin{array}{ll}
\coprod_{c \in \mathscr{S}_{1}}(T, \mathscr{T}) \rightarrow \mathrm{U}\left(T^{\prime} \otimes S^{\prime}, \mathscr{T}^{\prime} \otimes \mathscr{S}^{\prime}\right), & \coprod_{a \in \mathscr{T}_{1}}(S, \mathscr{S}) \rightarrow \mathrm{U}\left(T^{\prime} \otimes S^{\prime}, \mathscr{T}^{\prime} \otimes \mathscr{S}^{\prime}\right), \\
x \otimes c \mapsto f(x) \otimes g(c), & a \otimes y \mapsto f(a) \otimes g(y) .
\end{array}
$$


Taking the transpose morphisms in Prob, and using the universal property of the pushout (13) which is preserved by $\mathrm{F}$, we obtain a unique morphism $\mathrm{F}(T \square S) \rightarrow\left(T^{\prime} \otimes S^{\prime}, \mathscr{T}^{\prime} \otimes \mathscr{S}^{\prime}\right)$ of probs which is compatible with the $\varphi \otimes \psi$ equations, hence factors uniquely through a morphism

$$
f \otimes g:(T \otimes S, \mathscr{T} \otimes \mathscr{S}) \rightarrow\left(T^{\prime} \otimes S^{\prime}, \mathscr{T}^{\prime} \otimes \mathscr{S}^{\prime}\right)
$$

This defines a functor $-\otimes-:$ Pro $\times$ Pro $\rightarrow$ Prob.

Remark 5.8. When either $\varphi$ or $\psi$ is a unit, the equation $\varphi \otimes \psi$ holds automatically by the axioms of braidings. So $\varphi \otimes \psi$ is only non-trivial when both cells have rank 2 .

One can derive, as a consequence, that the monoid $\mathbb{N}$ is a "relative unit" for the tensor product, in the sense that the functors $\mathbb{N} \otimes-$ and $-\otimes \mathbb{N}$ are naturally isomorphic to F: Pro $\rightarrow$ Prob.

Example 5.9. We compute the tensor product Bialg $:=$ Mon $\otimes$ Mon $^{\mathrm{co}}$ of the theories of monoids and comonoids. Both Mon and $\mathrm{Mon}^{\mathrm{co}}$ are one-sorted, so Bialg is also one-sorted.

In fact, the indexed coproducts (12) are equal to Mon and $M o n^{\mathrm{co}}$, respectively, while $\sigma_{\leq 1}$ (Bialg) is isomorphic to $\mathbb{N}$, so the pushout (13) can be computed as

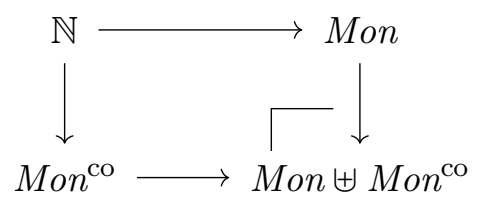

in Pro. The 2-cells in $M o n \uplus M o n^{\mathrm{co}}$ are freely generated by those of Mon and $M o n^{\mathrm{co}}$, modulo any equations that hold in the two factors separately: a model of $M o n \uplus M o n^{\mathrm{co}}$ is a pair of a monoid and a comonoid structure on the same object.

Finally, to obtain Bialg, we quotient $\mathrm{F}\left(M o n \uplus M o n^{\mathrm{co}}\right)$ by the $\varphi \otimes \psi$ equations. It suffices to let $\varphi$ and $\psi$ range over 2-cells that generate $M o n$ and $M_{o n}{ }^{\mathrm{co}}$, respectively, under composition.

An obvious choice is to take the unique maps $\mu:(2) \Rightarrow(1)$ and $\eta:(0) \Rightarrow(1)$ as generators of Mon, and their duals $\delta:(1) \Rightarrow(2)$ and $\varepsilon:(1) \Rightarrow(0)$ as generators of $\mathrm{Mon}^{\mathrm{co}}$. We may picture these as
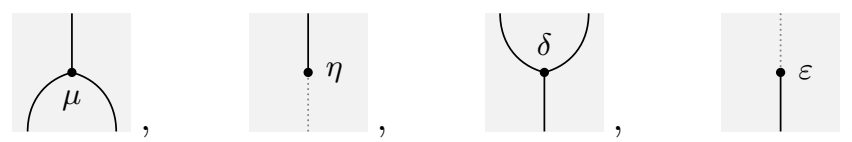
The four corresponding equations are

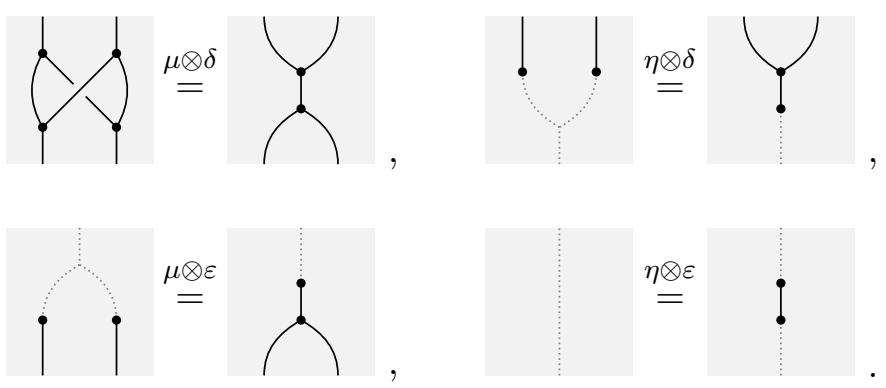

In a symmetric monoidal category, a pair of a monoid and a comonoid satisfying these equations is a bialgebra [Pir02]. In a braided monoidal category, this notion forks into two variants, distinguished by the use of braidings or inverse braidings, classified by Bialg and by Bialg ${ }^{*}=M_{o n}{ }^{\mathrm{co}} \otimes$ Mon, respectively.

Example 5.10. We compute the tensor product BrCMon $:=$ Mon $\otimes$ Mon. We proceed as in Example 5.9 to derive that BrCMon is the quotient of $\mathrm{F}($ Mon $\uplus$ Mon $)$ by the equations $\varphi \otimes \psi$ where $\varphi, \psi$ range over $\{\mu, \eta\}$.

Using different colours to distinguish cells from each copy of Mon, these can be pictured as

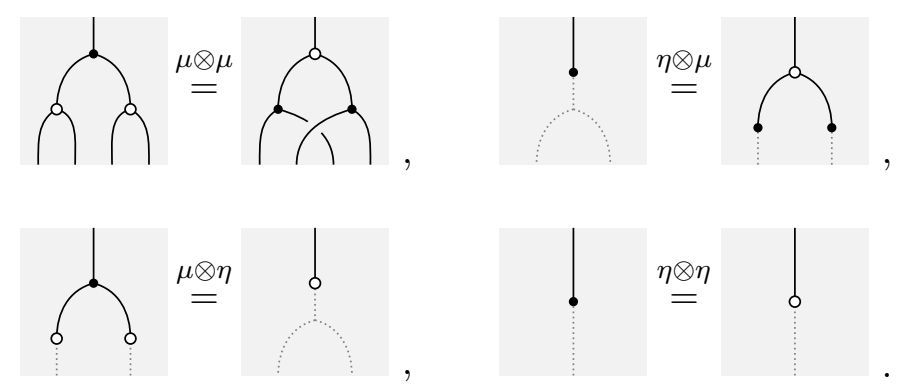

A model of Mon $\uplus$ Mon is a pair of monoid structures on the same object. It is a consequence of the Eckmann-Hilton argument, valid in every braided monoidal category, that a pair of monoid structures satisfying the equations $\mu \otimes \mu$ and $\eta \otimes \eta$ coincide with a single commutative monoid structure. The equations $\eta \otimes \mu$ and $\mu \otimes \eta$ are derivable from the rest.

We conclude that $B r C M o n$ is the braided monoidal theory of commutative monoids, whose reflection $\mathrm{r}(B r C M o n)$ is isomorphic to CMon. Dually, $M o n^{\mathrm{co}} \otimes M o n^{\mathrm{co}}$ is the braided monoidal theory of commutative comonoids.

5.11 (Tensor product of props). The tensor product $(T, \mathscr{T}) \otimes_{\mathbb{S}}(S, \mathscr{S})$ of two props $(T, \mathscr{T})$ and $(S, \mathscr{S})$ is the quotient of $\mathrm{r}(\mathrm{U}(T, \mathscr{T}) \otimes \mathrm{U}(S, \mathscr{S}))$ by the equations

$$
\sigma_{a, b} \otimes c=\sigma_{a \otimes c, b \otimes c}, \quad a \otimes \sigma_{c, d}=\sigma_{a \otimes c, a \otimes d}
$$


for all $a, b \in \mathscr{T}_{1}$ and $c, d \in \mathscr{S}_{1}$, where $\sigma_{a, b}$ and $\sigma_{c, d}$ are the original braidings of $T$ and $S$.

As shown in [HR15, Section 3], the tensor product of props is part of a symmetric monoidal closed structure on Prop, whose unit is the theory of permutations $\mathbb{S}$.

Example 5.12. Given a prop $(T, \mathscr{T})$, the tensor product $(T, \mathscr{T}) \otimes_{\mathbb{S}} C M o n^{\text {co }}$ is a cartesian prop, also known as a Lawvere theory. It is in fact the free cartesian prop on $(T, \mathscr{T})$ [Bae06].

Comment 5.13. The tensor product of props is compatible with the tensor product of pros in the sense that the diagram of functors

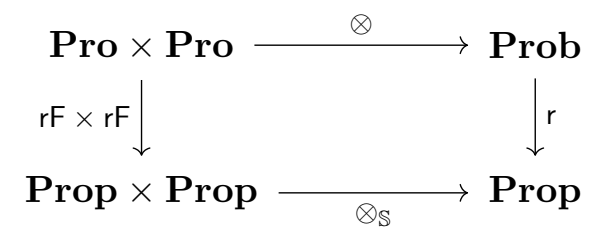

commutes up to natural isomorphism. The reason why this works is that, when $\varphi$ or $\psi$ is a braiding $\sigma_{a, b}$, the equation $\varphi \otimes \psi$ combined with (14) holds automatically in a prop. It follows that, while $\operatorname{UrF}(T, \mathscr{T}) \otimes \operatorname{UrF}(S, \mathscr{S})$ has additional generators and equations compared to $(T, \mathscr{T}) \otimes(S, \mathscr{S})$, these are all trivialised by the combined action of $r$ and (14).

This fact is specific to props and does not generalise to probs: the quotient of $\operatorname{UF}(T, \mathscr{T}) \otimes \operatorname{UF}(S, \mathscr{S})$ by (14) in Prob is not in general isomorphic to $(T, \mathscr{T}) \otimes(S, \mathscr{S})$. For example, the quotient of $\mathrm{U} \mathbb{B} \otimes \mathrm{UB}$ by $(14)$ is not isomorphic to $\mathbb{N} \otimes \mathbb{N} \simeq \mathbb{B}$. Indeed, if $\sigma_{1,1}:(2) \Rightarrow(2)$ is a braiding in $\mathbb{B}$, the equation $\sigma_{1,1} \otimes \sigma_{1,1}$ becomes

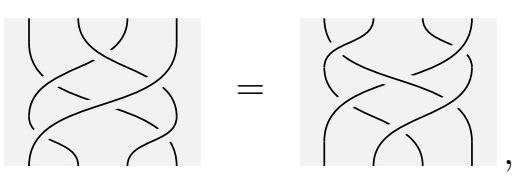

which does not hold in the braid group on 4 strands. This can be checked by considering the link diagrams
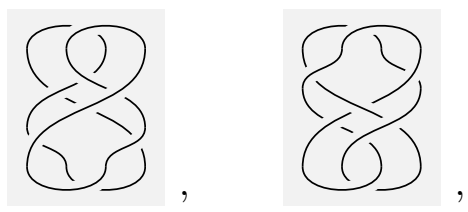
and observing that the first is an unlink while the second is not. On the other hand, the reflected equation

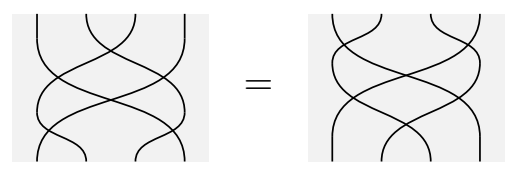

is valid in the theory of permutations.

As a consequence, there does not seem to be an interesting monoidal structure on Prob that generalises the one on Prop. Following the interpretation of the tensor product as a smash product, we believe that symmetric monoidal theories being closed under the tensor product is a consequence of symmetric monoidal structures being stable under smash products in the sense of stable homotopy theory.

Remark 5.14. As shown in [HR15, Proposition 40], the tensor product of props extends the Boardman-Vogt product of symmetric operads [BV06], in the sense that there is an embedding of the category of symmetric operads into the category of props which is strong monoidal with respect to the two monoidal structures.

Remark 5.15. The tensor product of pros is not symmetric. Up to the definition of $T \square S$ as the pushout (13), the construction of $(T, \mathscr{T}) \otimes(S, \mathscr{S})$ and of $(S, \mathscr{S}) \otimes(T, \mathscr{T})$ is, indeed, identical up to a change of notation. However, in the final quotient, the roles of $\sigma$ and $\sigma^{*}$, or braidings and inverse braidings, are reversed.

Nevertheless, this argument reveals a natural isomorphism between

$$
(T, \mathscr{T}) \otimes(S, \mathscr{S}) \quad \text { and } \quad((S, \mathscr{S}) \otimes(T, \mathscr{T}))^{*},
$$

where $-^{*}$ is the duality defined in $\S 2.21$. From this we can recover a symmetry for the tensor product of props.

5.2. The smash product of pointed diagrammatic sets

5.16 (Gray product). Let $P, Q$ be regular directed complexes. The Gray product $P \otimes Q$ of $P$ and $Q$ is the cartesian product $P \times Q$ of their underlying posets with the following orientation. Write $x \otimes y$ for a generic element of $P \otimes Q$. For all $x^{\prime}$ covered by $x$ in $P$ and all $y^{\prime}$ covered by $y$ in $Q$,

$$
\begin{aligned}
& o\left(x \otimes y \rightarrow x^{\prime} \otimes y\right):=o_{P}\left(x \rightarrow x^{\prime}\right), \\
& o\left(x \otimes y \rightarrow x \otimes y^{\prime}\right):=(-)^{\operatorname{dim}(x)} o_{Q}\left(y \rightarrow y^{\prime}\right),
\end{aligned}
$$

where $o_{P}$ and $o_{Q}$ are the orientations of $P$ and $Q$, respectively. 
As shown in $[\operatorname{Had} 20 \mathrm{~b}$, Section 2.2$], P \otimes Q$ is a regular directed complex. If $f: P \rightarrow P^{\prime}$ and $g: Q \rightarrow Q^{\prime}$ are maps of regular directed complexes, let $f \otimes g: P \otimes Q \rightarrow P^{\prime} \otimes Q^{\prime}$ have the cartesian product of $f$ and $g$ as underlying function. Then $f \otimes g$ is a map of regular directed complexes.

Gray products determine a monoidal structure on $\mathbf{D C} \mathbf{C p x ^ { \mathcal { R } }}$ whose unit is the terminal object 1 .

5.17. The monoidal structure on $\mathbf{D C p} \mathbf{p x}^{\mathcal{R}}$ restricts to a monoidal structure on $\odot$, which, by Day's theory [Day70], extends along the Yoneda embedding to a monoidal biclosed structure on $\odot$ Set.

Explicitly, let $X$ and $Y$ be diagrammatic sets. The Gray product $X \otimes Y$ of $X$ and $Y$ is the colimit in $\odot$ Set of the diagram

$$
\odot / X \times \odot / Y \stackrel{\text { dom } \times \text { dom }}{\longrightarrow} \odot \times \odot \stackrel{\otimes}{\longrightarrow} \odot \longleftrightarrow \odot \text { Set }
$$

where $\odot / X$ is the category whose objects are cells $x: U \rightarrow X$ and morphisms from $x: U \rightarrow X$ to $y: V \rightarrow X$ are commutative triangles

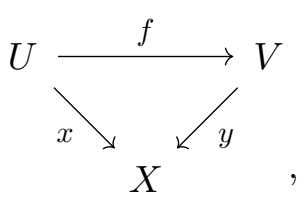

while dom sends such a triangle to the map $f: U \rightarrow V$ in $\odot$.

In particular, for each pair of cells $x: U \rightarrow X$ and $y: V \rightarrow Y$, the image of the pair $(x, y)$ through the diagram $(16)$ is $U \otimes V$, so we obtain a morphism $U \otimes V \rightarrow X \otimes Y$ to the colimit, that is, a cell of shape $U \otimes V$ in $X \otimes Y$. This is the cell $x \otimes y$ obtained as the Gray product of $x$ and $y$ in $\odot$ Set.

Remark 5.18. The dimensions of cells add under the Gray product, that is, if $x$ is an $n$-cell and $y$ is an $m$-cell, then $x \otimes y$ is an $(n+m)$-cell.

Remark 5.19. The Gray product is not the cartesian product in $\odot$ Set. However, the monoidal unit is the terminal object, which gives us "projection" morphisms $X \otimes Y \rightarrow X$ and $X \otimes Y \rightarrow Y$. These send a cell $x \otimes y$ of shape $U \otimes V$ to $p_{1} ; x$ and $p_{2} ; y$, respectively, where $p_{1}: U \otimes V \rightarrow U$ and $p_{2}: U \otimes V \rightarrow V$ are projections in $\odot$.

Comment 5.20. We use string diagrams to give some intuition about cells $x \otimes y$ of shape $U \otimes V$ in low dimension; in the pictures, we write $x y$ for $x \otimes y$. First of all, if $x$ or $y$ is a 0 -cell, then $U \otimes V$ is isomorphic to $V$ or $U$, respectively, and $x \otimes y$ has the same dimension and shape as $y$ or $x$. 
Let $a: x^{-} \Rightarrow x^{+}$be a 1-cell in $X$ and $c: y^{-} \Rightarrow y^{+}$a 1-cell in $Y$. Then $a \otimes c$ is a 2-cell of the form

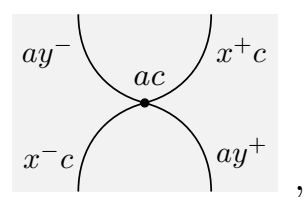

that is, it is of type $\left(x^{-} \otimes c\right) \#_{0}\left(a \otimes y^{+}\right) \Rightarrow\left(a \otimes y^{-}\right) \#_{0}\left(x^{+} \otimes c\right)$ in $X \otimes Y$.

Next, let $\varphi: a_{1} \#_{0} \ldots \#_{0} a_{n} \Rightarrow b_{1} \#_{0} \ldots \#_{0} b_{m}$ be a 2-cell in $X$, and let $\psi: c_{1} \#_{0} \ldots \#_{0} c_{p} \Rightarrow d_{1} \#_{0} \ldots \#_{0} d_{q}$ be a 2 -cell in $Y$. Then $\varphi \otimes c$ is a 3 -cell of the form

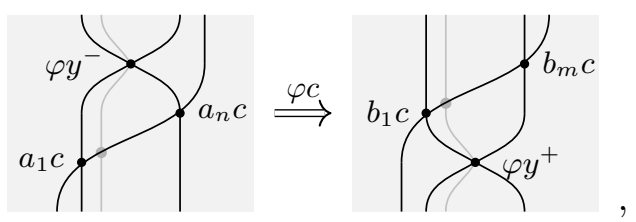

while $a \otimes \psi$ is a 3 -cell of the form

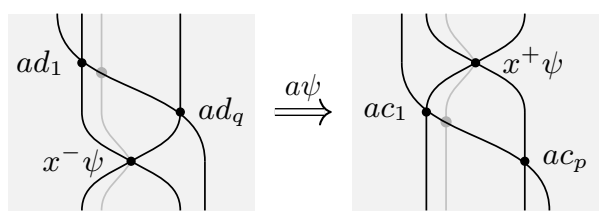

in $X \otimes Y$. It is useful to think of these as sliding moves: $\varphi \otimes c$ slides a 2-cell in the fibre of $\varphi$ left-to-right, top-to-bottom past a 1-cell in the fibre of $c$, while $a \otimes \psi$ slides a 2-cell in the fibre of $\psi$ left-to-right, bottom-to-top past a 1-cell in the fibre of $a$.

Next, we consider the 4-cell $\varphi \otimes \psi$; to simplify, we depict $\varphi$ and $\psi$ as if they had only 2 inputs and 2 outputs each. Then $\partial^{-}(\varphi \otimes \psi)$ is the 3 -diagram

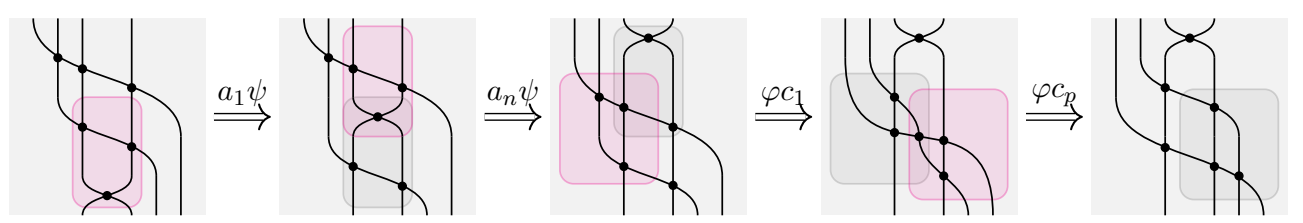

where the sequence of sliding moves $a_{1} \otimes \psi, \ldots, a_{n} \otimes \psi$ is followed by the sequence $\varphi \otimes c_{1}, \ldots, \varphi \otimes c_{p}$, while $\partial^{+}(\varphi \otimes \psi)$ is the 3 -diagram

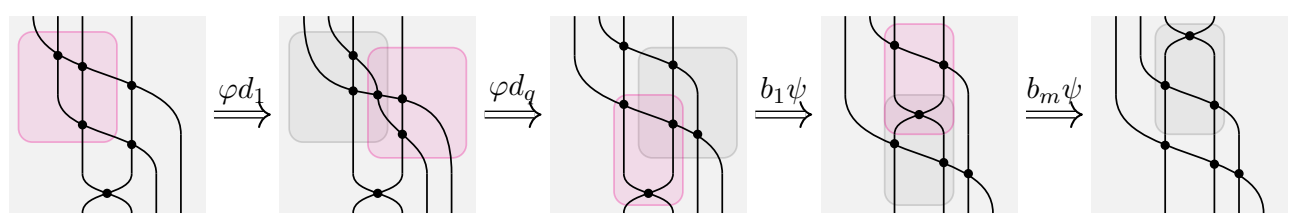

where the sequence of sliding moves $\varphi \otimes d_{1}, \ldots, \varphi \otimes d_{q}$ is followed by the sequence $b_{1} \otimes \psi, \ldots, b_{m} \otimes \psi$. In the case $n, m, p, q=2$, one can recognise the two sides of the Zamolodchikov tetrahedron equation [KV94]. 
Next, let $\rho$ be a 3-cell in $X$ and consider the 4-cell $\rho \otimes c$. To simplify, we depict $\rho$ as if it were of type $\varphi \Rightarrow \varphi^{\prime}$ where $\varphi$ and $\varphi^{\prime}$ are both 2-cells. Then $\partial^{-}(\rho \otimes c)$ has the form

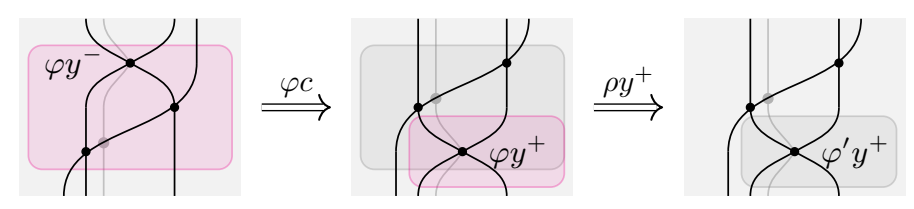

while $\partial^{+}(\rho \otimes c)$ has the form

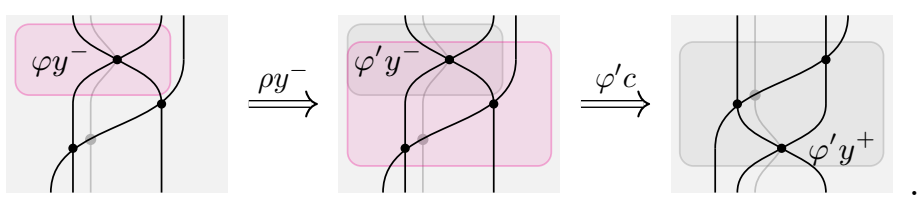

Dually, if $\tau: \psi \Rightarrow \psi^{\prime}$ is a 3-cell in $Y, \partial^{-}(a \otimes \tau)$ has the form

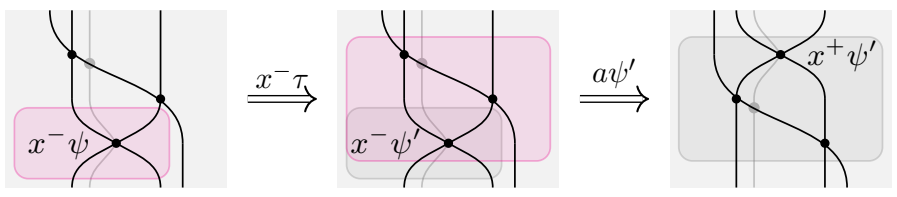

while $\partial^{+}(a \otimes \tau)$ has the form

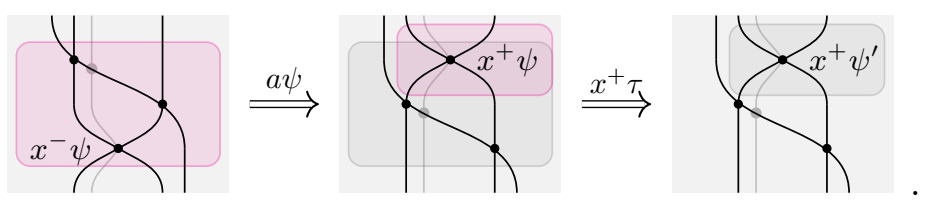

5.21 (Pointed diagrammatic set). A pointed diagrammatic set is a diagrammatic set $X$ together with a distinguished 0 -cell $\bullet: 1 \rightarrow X$, the basepoint.

A morphism $f:\left(X, \bullet_{X}\right) \rightarrow\left(Y, \bullet_{Y}\right)$ of pointed diagrammatic sets is a morphism $f: X \rightarrow Y$ such that $f\left(\bullet_{X}\right)=\bullet_{Y}$. With their morphisms, pointed diagrammatic sets form a category $\odot$ Set.

5.22. The obvious forgetful functor $\odot$ Set. $\rightarrow \odot$ Set has a left adjoint sending a diagrammatic set $X$ to the coproduct $X+1$, pointed with the inclusion of 1 into the coproduct.

The terminal object 1 of $\odot$ Set, pointed with its only 0 -cell, is a zero object in $\odot$ Set., both terminal and initial.

5.23 (Wedge sum). The wedge sum of two pointed diagrammatic sets $\left(X, \bullet_{X}\right)$ and $\left(Y, \bullet_{Y}\right)$ is the pointed diagrammatic set $(X \vee Y, \bullet)$ where

1. $X \vee Y$ is the quotient of $X+Y$ by the equation $\bullet_{X}=\bullet_{Y}$, and 
2. $\bullet$ is the result of the identification of $\bullet X$ and $\bullet Y$.

5.24 (Smash product). Let $\left(X, \bullet_{X}\right)$ and $(Y, \bullet Y)$ be pointed diagrammatic sets. There is an inclusion $X \vee Y \hookrightarrow X \otimes Y$ defined by

$$
x \mapsto x \otimes \bullet_{Y}, \quad y \mapsto \bullet X \otimes y
$$

on cells in $X$ and $Y$, respectively.

The smash product of $\left(X, \bullet_{X}\right)$ and $\left(Y, \bullet_{Y}\right)$ is the pointed diagrammatic set $(X \otimes Y, \bullet)$ obtained from the pushout diagram

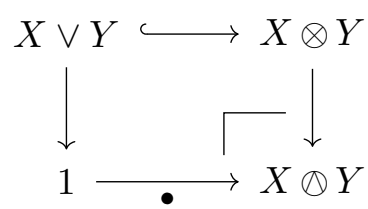

in $\odot$ Set (the "quotient of $X \otimes Y$ by the subspace $X \vee Y$ ").

The smash product is part of a monoidal structure on $\odot$ Set., whose unit is the diagrammatic set $1+1$, pointed with one of the coproduct inclusions, and all structural isomorphisms are derived from those of the Gray product.

Comment 5.25. The smash product of pointed diagrammatic sets is a "directed" counterpart to the smash product of pointed topological spaces, with the Gray product playing the rôle of the cartesian product of spaces.

The formal correspondence between definitions is made concrete through the geometric realisation of diagrammatic sets [Had20b, §8.38]. This functor $|-|: \odot$ Set $\rightarrow$ cgHaus sends 0-cells in a diagrammatic set to points in a space, so it lifts to a functor

$$
|-|: \odot \text { Set• } \rightarrow \text { cgHaus }
$$

to the category of pointed compactly generated Hausdorff spaces and pointed continuous maps.

We claim that this functor sends smash products in $\odot$ Set. to smash products in cgHaus., that is, it is strong monoidal with respect to the two monoidal structures.

Proof. On regular atoms, $|-|$ is defined as the forgetful functor from $\odot$ to the category of posets and order-preserving maps, followed by the simplicial nerve of posets, followed by the geometric realisation of simplicial sets. The first sends Gray products to cartesian products and the other two preserve finite products. Thus $|U \otimes V| \simeq|U| \times|V|$ naturally in $U$ and $V$.

Both Gray products in $\odot$ Set and products in cgHaus are part of a biclosed monoidal structure, so they preserve colimits separately in each variable. Since 
$|-|$, a left adjoint functor, also preserves colimits, we can extend to an isomorphism $|X \otimes Y| \simeq|X| \times|Y|$ natural in the diagrammatic sets $X$ and $Y$.

Finally, $|-|$ also preserves the terminal object, so it sends the colimit diagrams that define wedge sums and smash products in $\odot$ Set. to the colimit diagrams that define them in cgHaus.

5.26. Like the smash product of pointed spaces, the smash product of pointed diagrammatic sets is part of a biclosed structure on $\odot$ Set.

Left homs and right homs can be computed by a formal argument. If $\left(X, \bullet_{X}\right) \multimap\left(Y, \bullet_{Y}\right)$ is a right hom in $(\odot$ Set., $\otimes, 1+1)$, cells of shape $U$ in its underlying diagrammatic set correspond to pointed morphisms from $U+1$ to $\left(X, \bullet_{X}\right) \multimap\left(Y, \bullet_{Y}\right)$, which correspond to pointed morphisms from $(X, \bullet X) \oplus(U+1)$ to $\left(Y, \bullet_{Y}\right)$.

Now $X \otimes(U+1)$ is isomorphic to the quotient of $X \otimes U$ by the subspace $\{\bullet X\} \otimes U$. By the universal property of this quotient, we conclude that there is a bijection between

1. cells of shape $U$ in $(X, \bullet X) \multimap\left(Y, \bullet_{Y}\right)$ and

2. morphisms $X \otimes U \rightarrow Y$ which send $\{\bullet X\} \otimes U$ to $\left\{\bullet_{Y}\right\}$.

Similarly, cells of shape $U$ in the left hom $\left(Y, \bullet_{Y}\right) \circ-\left(X, \bullet_{X}\right)$ correspond bijectively to morphisms $U \otimes X \rightarrow Y$ sending $U \otimes\left\{\bullet_{X}\right\}$ to $\left\{\bullet_{Y}\right\}$.

In particular, the 0-cells in both the left and the right hom are the pointed morphisms from $\left(X, \bullet_{X}\right)$ to $\left(Y, \bullet_{Y}\right)$. The basepoint, classified by the only morphism from the zero object, is the constant morphism $X \mapsto \bullet_{Y}$.

Comment 5.2\%. From the string diagram of a cell in $X \otimes Y$, it is easy to obtain a picture of the same cell in $X \otimes Y$ : we simply need to identify every cell of the form $x \otimes \bullet_{Y}$ or $\bullet_{X} \otimes y$ and shape $U$ with the cell !; $\bullet$ of the same shape, where !: $U \rightarrow 1$ is the unique map to the terminal object.

We will depict all such 1-cells as dotted wires, and all such 2-cells as dotless nodes, which is consistent with our convention for units and unitors in the nerve of a pro. For example, if $X$ and $Y$ have a single 0-cell, then any 3-cell of the form $\varphi \otimes c$ as in (17) or $a \otimes \psi$ as in (18) becomes

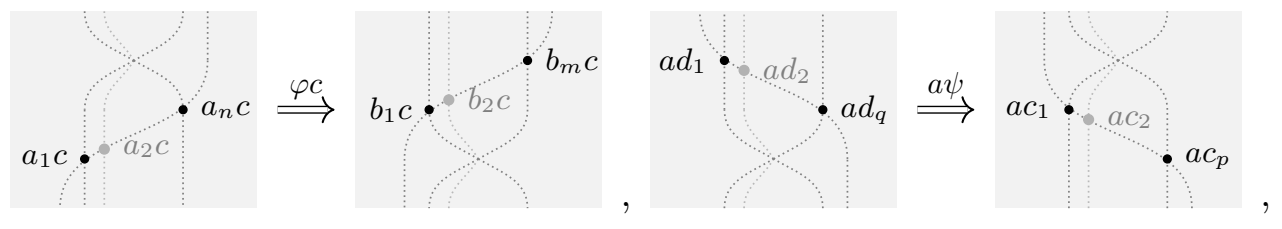

respectively, in $X \oplus Y$. 


\subsection{Comparison of the constructions}

We are ready to state our main theorem.

5.28. Let $(T, \mathscr{T})$ be a pro. Its diagrammatic nerve $\mathrm{N}(T, \mathscr{T})$ has a single 0-cell, so it is canonically pointed, and every morphism in the image of $\mathrm{N}$ trivially preserves the basepoint. Thus N, restricted to Pro, lifts uniquely to a functor N : Pro $\rightarrow \odot$ Set.

Theorem 5.29 - The diagram of functors

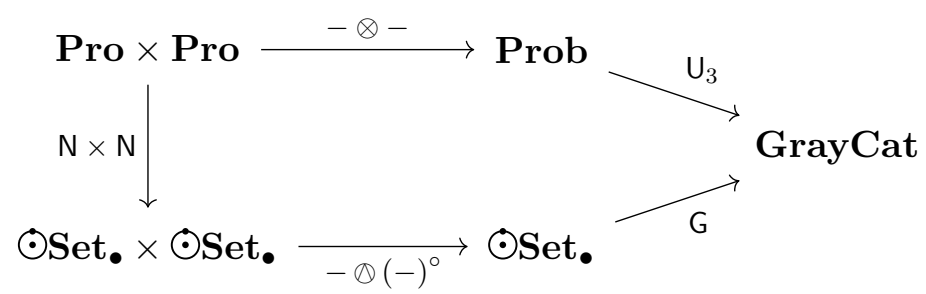

commutes up to natural isomorphism.

Comment 5.30. The form of Theorem 5.29 does not suggest, at first sight, that the smash product of pointed diagrammatic sets subsumes and generalises the tensor product of props. Nevertheless, we argue that this is essentially the case.

First of all, since $\mathrm{U}_{3}$ is pseudomonic by Remark 2.37, if $\mathrm{G} X$ is isomorphic to $\mathrm{U}_{3}(T, \mathscr{T})$ for some prob $(T, \mathscr{T})$, then this prob is essentially unique. It follows that, on the image of $\mathrm{N}-\otimes(\mathrm{N}-)^{\circ}$, we can lift $\mathrm{G}$ to a functor with codomain Prob, and compute the tensor product of two pros through the lower leg of the diagram. In this sense, the smash product on $\odot$ Set• strictly subsumes the "external" tensor product of pros.

From the tensor product of pros, we can recover the tensor product of props via a universal characterisation in Prop, independent of the specific construction. If $(T, \mathscr{T})$ and $(S, \mathscr{S})$ are two props, we have families of morphisms

$$
\begin{aligned}
& \mathrm{id}_{T} \otimes c: \mathrm{FU}(T, \mathscr{T}) \rightarrow \mathrm{U}(T, \mathscr{T}) \otimes \mathrm{U}(S, \mathscr{S}), \\
& a \otimes \operatorname{id}_{S}: \mathrm{FU}(S, \mathscr{S}) \rightarrow \mathrm{U}(T, \mathscr{T}) \otimes \mathrm{U}(S, \mathscr{S})
\end{aligned}
$$

in Prob indexed by $c \in \mathscr{S}_{1}$ and $a \in \mathscr{T}_{1}$, where $c: \mathbb{N} \rightarrow \mathrm{U}(S, \mathscr{S})$ and $a: \mathbb{N} \rightarrow \mathrm{U}(T, \mathscr{T})$ send the generating 1-cell of $\mathbb{N}$ to $c$ and $a$, respectively; here we use the fact that $-\otimes \mathbb{N}$ and $\mathbb{N} \otimes-$ are naturally isomorphic to $\mathrm{F}$. Then $(T, \mathscr{T}) \otimes_{\mathbb{S}}(S, \mathscr{S})$ is the pushout

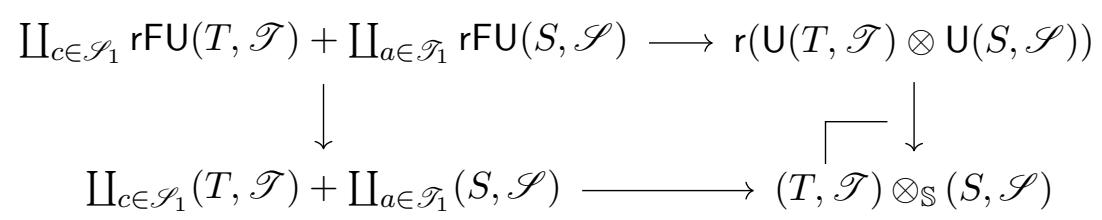


in Prop, where the top leg is obtained universally from the family of morphisms $\left\{\mathrm{r}\left(\mathrm{id}_{T} \otimes c\right), \mathrm{r}\left(a \otimes \mathrm{id}_{S}\right) \mid c \in \mathscr{S}_{1}, a \in \mathscr{T}_{1}\right\}$ and the left leg from the counit of the adjunction between $r F$ and $\mathrm{U}$.

5.31 (Skeleta of diagrammatic sets). For each $n \in \mathbb{N}+\{-1\}$, the restriction functor $\odot$ Set $\rightarrow \operatorname{PSh}\left(\odot_{n}\right)$ has a left adjoint; let $\sigma_{\leq n}$ be the comonad induced by this adjunction.

The $n$-skeleton of a diagrammatic set $X$ is the counit $\sigma_{\leq n} X \rightarrow X$. For all $k \leq n$, the $k$-skeleton factors uniquely through the $n$-skeleton of $X$. By a standard argument $X$ is the colimit of the sequence of its skeleta.

Proof of Theorem 5.29. Let $(T, \mathscr{T})$ and $(S, \mathscr{S})$ be two pros and let $(X, \bullet X)$ and $\left(Y, \bullet_{Y}\right)$ be equal to $\mathrm{N}(T, \mathscr{T})$ and $(\mathrm{N}(S, \mathscr{S}))^{\circ}$, respectively.

As seen in $\S 5.17, X \otimes Y$ is the colimit of a diagram of atoms $U \otimes V$ indexed by pairs of cells $x: U \rightarrow X$ and $y: V \rightarrow Y$, which are transposes of morphisms $x: \mathrm{P} U \rightarrow(T, \mathscr{T})$ and $y: \mathrm{P} V^{\circ} \rightarrow(S, \mathscr{S})$ in Pro. The smash product $X \otimes Y$ is then the colimit of this diagram extended with a morphism $U \otimes V \rightarrow 1$ for all atoms $U \otimes V$ indexed by $\left(x, \bullet_{Y}\right)$ or by $(\bullet X, y)$.

Each diagrammatic set is the colimit of the sequence of its skeleta, and this colimit is preserved by smash products separately in each variable. Because colimits commute with colimits, we can compute $X \otimes Y$ in steps, increasing $i$ and $j$ separately in $\sigma_{\leq i} X \otimes \sigma_{\leq j} Y$. This corresponds to restricting the indexing category to pairs $(x, y)$ with $\operatorname{dim}(x) \leq i$ and $\operatorname{dim}(y) \leq j$.

The functor G: $\odot$ Set $\rightarrow$ GrayCat, a left adjoint, preserves colimits, and we know how to explicitly compute $\mathrm{G}$ on atoms. We will use this to compute $\mathrm{G}(X \otimes Y)$.

- Let $i=0$ or $j=0$. Since the only 0-cell in $X$ and $Y$ is their basepoint, both $\sigma_{\leq 0} X \otimes Y$ and $X \otimes \sigma_{\leq 0} Y$ are isomorphic to the terminal diagrammatic set. Their image through $\mathrm{G}$ is the terminal Gray-category with one 0-cell and no cells of higher rank.

- Let $i=j=1$. The 1 -cells in $X$ that do not factor through $\sigma_{\leq 0} X$ correspond bijectively to generating 1-cells $a \in \mathscr{T}_{1}$, and the 1-cells in $Y$ that do not factor through $\sigma_{\leq 0} Y$ to generating 1-cells $c \in \mathscr{S}_{1}$.

The boundary of $a \otimes c$ contains only 1-cells of the form !; $\bullet$, which G sends to units on $\bullet$. Through $\mathrm{G}$, then, $a \otimes c$ becomes a 2-cell of type $\varepsilon \bullet \Rightarrow \varepsilon \bullet$. Thus $\mathrm{G}\left(\sigma_{\leq 1} X \otimes \sigma_{\leq 1} Y\right)$ has a single 0-cell, a single 1-cell, and its 2-cells are freely generated by the $a \otimes c$ : this makes it a prob in the sense of $\S 2.35$, isomorphic to $\mathrm{F}\left(\sigma_{\leq 1}(T \otimes S)\right)$. This structure of prob will be inherited by $\mathrm{G}\left(\sigma_{\leq i} X \otimes \sigma_{\leq j} Y\right)$ for all higher $i, j$. 
- We fix $j=1$ and increase $i$; observe that we can stop at $i=3$, since for $i>3$ we only include cells of dimension $>4$, whose contribution through $\mathrm{G}$ is trivial.

Let $c \in \mathscr{S}_{1}$. Each 2-cell $\varphi:\left(a_{1}, \ldots, a_{n}\right) \Rightarrow\left(b_{1}, \ldots, b_{m}\right)$ in $T$ contributes a 3-cell $\varphi \otimes c$ in $\sigma_{\leq 2} X \otimes \sigma_{\leq 1} Y$; any other 2-cell in $X$ factors through one of this form, so it does not give a contribution. We can read the form of $\varphi \otimes c$ from Comment 5.27: unravelling the definition of G on 3-atoms, we see that it sends $\varphi \otimes c$ to a 3-cell of type

$$
\left(a_{1} \otimes c\right) \#_{1} \ldots \#_{1}\left(a_{n} \otimes c\right) \Rightarrow\left(b_{1} \otimes c\right) \#_{1} \ldots \#_{1}\left(b_{m} \otimes c\right) .
$$

Extending along colimits, a 2-diagram $x \#_{k} y$ in $X$ induces a diagram $(x \otimes c) \#_{k+1}(y \otimes c)$ in $\mathrm{G}\left(\sigma_{\leq 2} X \otimes \sigma_{\leq 1} Y\right)$ for each $k \in\{0,1\}$.

From Proposition 4.6, we know that 3 -cells $\rho$ in $X$ exhibit all and only the equations of diagrams $x=y$ that hold in $T$. Reading the form of $\rho \otimes c$ from Comment 5.20, we see that the only part surviving both the smash product quotient and $\mathrm{G}$ is an equation between the composites of $x \otimes c$ and $y \otimes c$. For each $c \in \mathscr{S}_{1}$, then, we can define a morphism of probs

$$
-\otimes c: \mathrm{F}(T, \mathscr{T}) \rightarrow \mathrm{G}\left(X \otimes \sigma_{\leq 1} Y\right)
$$

by $x \mapsto x \otimes c$ on cells in $T$, extending universally to the free prob, and prove that it is injective. Moreover, the family of the $-\otimes c$ is jointly surjective and only overlaps on $\bullet$. We conclude that there is an isomorphism between $\mathrm{G}\left(X \otimes \sigma_{\leq 1} Y\right)$ and

$$
\coprod_{c \in \mathscr{S}_{1}} \mathrm{~F}(T, \mathscr{T}) \simeq \mathrm{F}\left(\coprod_{c \in \mathscr{S}_{1}}(T, \mathscr{T})\right),
$$

the coproducts being in Prob and Pro, respectively.

- The case where we fix $i=1$ and increase $j$ is dual, with a subtlety due to the way Gray products change orientations in their second factor depending on the dimension of the first factor. Since we defined $Y$ to be the dual of $\mathrm{N}(S, \mathscr{S})$, each 2-cell $\psi:\left(c_{1}, \ldots, c_{p}\right) \Rightarrow\left(d_{1}, \ldots, d_{q}\right)$ corresponds to a 2-cell

$$
\psi: d_{q} \#_{0} \ldots \#_{0} d_{1} \Rightarrow c_{p} \#_{0} \ldots \#_{0} c_{1}
$$

in $Y$, which for each $a \in \mathscr{T}_{1}$ contributes a 3-cell $a \otimes \psi$ in $\sigma_{\leq 1} X \otimes \sigma_{\leq 2} Y$. By inspection of the shape of $a \otimes \psi$ in Comment 5.27, we see that $\mathrm{G}$ sends it to a 3-cell of type

$$
\left(a \otimes c_{1}\right) \#_{1} \ldots \#_{1}\left(a \otimes c_{p}\right) \Rightarrow\left(a \otimes d_{1}\right) \#_{1} \ldots \#_{1}\left(a \otimes d_{q}\right),
$$


which matches the original orientation of $\psi$ in $S$. Proceeding as before, then, we construct an isomorphism between $\mathrm{G}\left(\sigma_{\leq 1} X \otimes Y\right)$ and

$$
\coprod_{a \in \mathscr{T}_{1}} \mathrm{~F}(S, \mathscr{S}) \simeq \mathrm{F}\left(\coprod_{a \in \mathscr{T}_{1}}(S, \mathscr{S})\right) .
$$

It follows that $\mathrm{G}\left(\left(X \otimes \sigma_{\leq 1} Y\right) \cup\left(\sigma_{\leq 1} X \otimes Y\right)\right)$ can be computed as the pushout

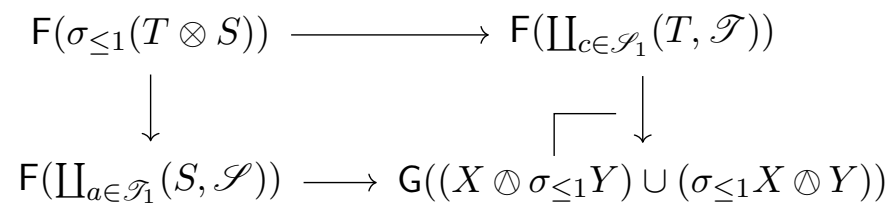

in Prob, isomorphic to $\mathrm{F}(T \square S)$.

- Finally, let $i=j=2$; increasing either $i$ or $j$ beyond 2 only includes cells of dimension $>4$, whose contribution is trivial.

Each pair of a 2-cell $\varphi:\left(a_{1}, \ldots, a_{n}\right) \Rightarrow\left(b_{1}, \ldots, b_{m}\right)$ in $T$ and a 2-cell $\psi:\left(c_{1}, \ldots, c_{p}\right) \Rightarrow\left(d_{1}, \ldots, d_{q}\right)$ in $S$ contributes a 4 -cell $\varphi \otimes \psi$ to $X \otimes Y$, and any other pair factors through one of this form.

Remember that the orientation of $\psi$ is reversed in $Y$. Reading the form of $\varphi \otimes \psi$ from Comment 5.20 and unravelling the definition of $\mathrm{G}$ on 4-atoms, we find that the boundaries of $\varphi \otimes \psi$ are mapped by $\mathrm{G}$ to the two sides of the $\varphi \otimes \psi$ equation in $\mathrm{F}(T \square S)$.

We conclude that $\mathrm{G}(X \otimes Y)$ with its unique prob structure is isomorphic to $(T, \mathscr{T}) \otimes(S, \mathscr{S})$. It is straightforward to check that the isomorphism is natural in $(T, \mathscr{T})$ and $(S, \mathscr{S})$.

Example 5.32. We compute an equation of the prob Bialg $=$ Mon $\otimes M^{c o}$ through $\odot$ Set., to illustrate how it arises from a 4-cell in the smash product of $X:=\mathrm{N}(M o n)$ and $Y:=\mathrm{N}\left(M o n^{\mathrm{co}}\right)^{\circ}$.

The two generating 1-cells of $M o n$ and $M o n^{\text {co }}$ produce a 2-cell $1 \otimes 1$ in $X \otimes Y$. From the generator $\mu:(2) \Rightarrow(1)$ of $M o n$, we obtain a 3 -cell $\mu \otimes 1$ in $X \otimes Y$. Because $1 \otimes 1$ is the only non-degenerate 2-cell appearing in the boundary of $\mu \otimes 1$, we may informally picture this 3 -cell as a string diagram in 3 -dimensional space, "tracing the history" of the various copies of $1 \otimes 1$ :

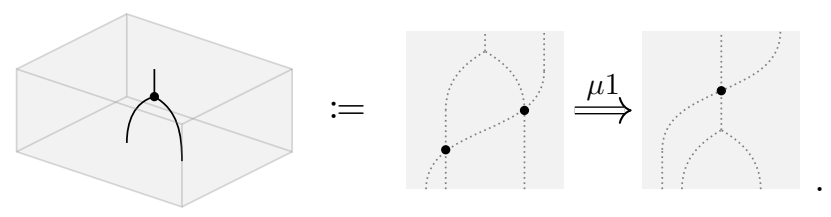


Similarly, from the generator $\delta:(1) \Rightarrow(2)$ of $M o n^{\text {co }}$, we obtain a 3 -cell $1 \otimes \delta$ of the form

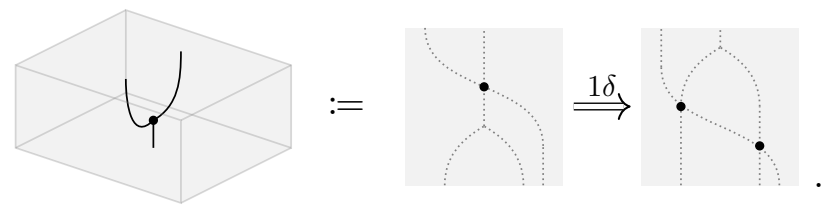

Now $\partial^{-}(\mu \otimes \delta)$ has the form

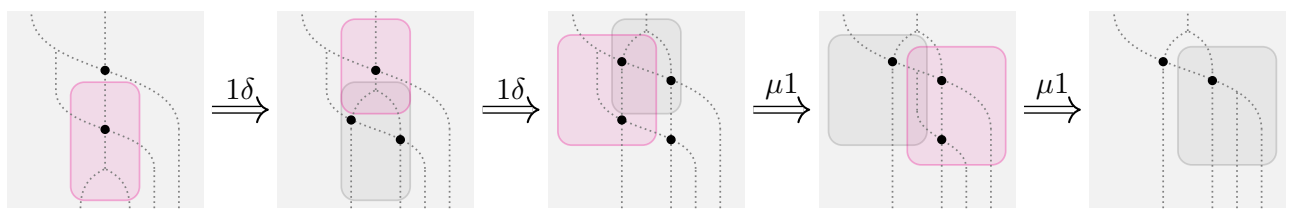

while $\partial^{+}(\mu \otimes \delta)$ has the form

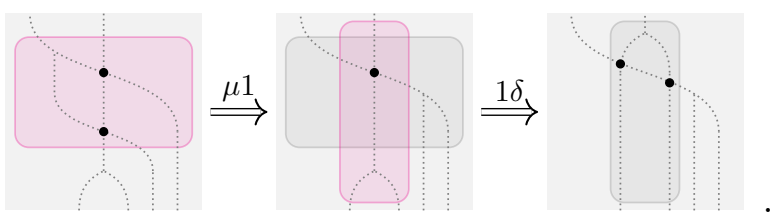

In 3-dimensional string diagrams, we may picture $\mu \otimes \delta$ as
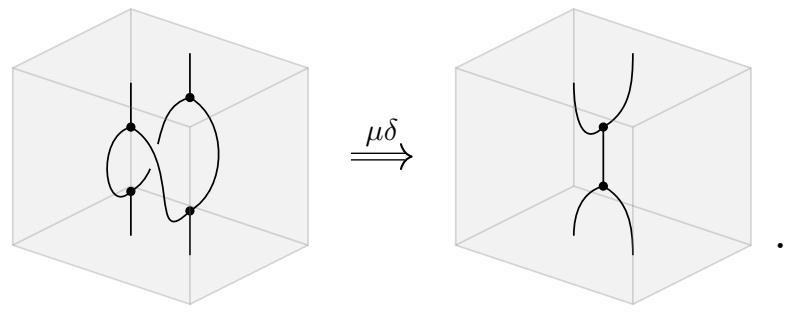

If we also "trace the history" of the dotted wires to produce surface diagrams in the style of [VD19], we recover, up to a deformation, the "intersecting surfaces" picture (2).

The picture of the equation $\mu \otimes \delta$ in Example 5.9 can be interpreted as a planar projection of (19). Technically, the single instance of a braiding in this equation arises, by definition of $\mathrm{G}$, from the fact that the input 2-cells of the first instance of $\mu \otimes 1$ in $\partial^{-}(\mu \otimes \delta)$ are not consecutive in the normal 1-order on the overall 2-diagram.

\section{HigheR-DimENSIONAL CELLS}

Having established that the smash product of pointed diagrammatic sets generalises the tensor product of pros, we briefly explore the potential of this generalisation in higher-dimensional universal algebra and rewriting. 
6.1 (Diagrammatic complex). A diagrammatic complex is a diagrammatic set $X$ together with a set $\mathscr{X}=\sum_{n \in \mathbb{N}} \mathscr{X}_{n}$ of generating cells such that, for all $n \in \mathbb{N}$

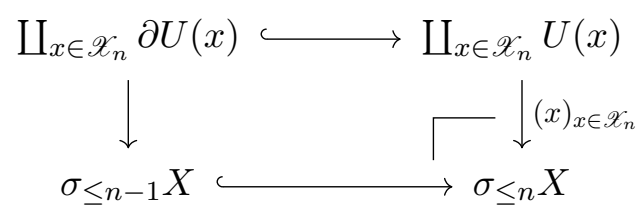

is a pushout in $\odot$ Set. Here $U(x)$ denotes the shape of the cell $x$.

Remark 6.2. It follows from the results of [Had20b, Section 8.3] that the geometric realisation of diagrammatic sets sends a diagrammatic complex $(X, \mathscr{X})$ to a CW complex with one cell for each generating cell in $\mathscr{X}$.

Proposition 6.3 - Let $(X, \mathscr{X})$ and $(Y, \mathscr{Y})$ be diagrammatic complexes. Then $X \otimes Y$ is a diagrammatic complex with

$$
(\mathscr{X} \otimes \mathscr{Y})_{n}:=\sum_{k=0}^{n}\left\{x \otimes y \mid x \in \mathscr{X}_{k}, y \in \mathscr{Y}_{n-k}\right\} .
$$

Proof. Essentially the same as [Had17, Theorem 1.35], replacing "polygraph" with "diagrammatic complex" and "globe" with "regular atom".

Remark 6.4. A straightforward consequence: the smash product $X \oplus Y$ of pointed diagrammatic complexes $\left(X, \mathscr{X}, \bullet_{X}\right)$ and $\left(Y, \mathscr{Y}, \bullet_{Y}\right)$ is a pointed diagrammatic complex whose generating cells are $\bullet$ and the pairs $x \otimes y$ with $x \in \mathscr{X} \backslash\left\{\bullet_{X}\right\}$ and $y \in \mathscr{Y} \backslash\left\{\bullet_{Y}\right\}$.

6.5 (Diagrammatic presentation). Let $(T, \mathscr{T})$ be a bicoloured pro. A presentation of $(T, \mathscr{T})$ is a diagrammatic complex $(X, \mathscr{X})$ such that $\mathrm{P} X$ is isomorphic to $(T, \mathscr{T})$.

Similarly let $(T, \mathscr{T})$ be a prob. A presentation of $(T, \mathscr{T})$ is a diagrammatic complex $(X, \mathscr{X})$ such that $\mathrm{G} X$ is isomorphic to $\mathrm{U}_{3}(T, \mathscr{T})$.

Example 6.6. The pro of monoids Mon admits the following presentation $(X, \mathscr{X})$. To begin, $\mathscr{X}_{0}$ contains a single 0 -cell $\bullet$ and $\mathscr{X}_{1}$ a single 1 -cell $1: \bullet \Rightarrow \bullet$. Next, $\mathscr{X}_{2}$ contains a 2 -cell $\mu: 1 \#_{0} 1 \Rightarrow 1$ and a 2 -cell $\eta: \varepsilon \bullet \Rightarrow 1$, which we picture as
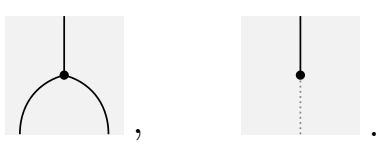

Finally, $\mathscr{X}_{3}$ contains 3 -cells $\alpha, \lambda, \rho$ of the form
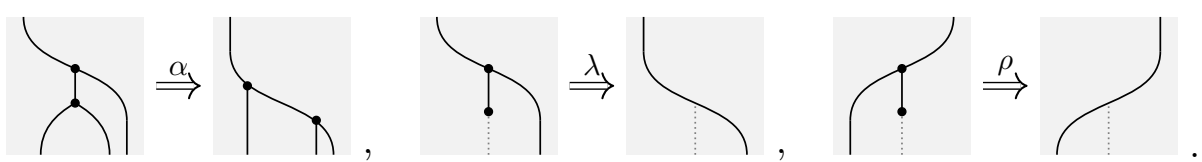
6.7. Unless a pro $(T, \mathscr{T})$ embodies a free monoidal theory, a presentation $(X, \mathscr{X})$ contains some generating 3-cells, exhibiting equations in $\mathrm{P} X$. Higherdimensional generators have no effect on the presented pro, as $\mathrm{P}$ turns them into trivial "equations of equations".

For presentations of a prob, the same statement applies shifted by one dimension: generating 4-cells exhibit equations, while higher-dimensional generators are trivialised by $\mathrm{G}$.

For the purpose of computing a tensor product of pros, we can replace the nerves of pros $(T, \mathscr{T}),(S, \mathscr{S})$ with any pair of presentations $(X, \mathscr{X}),(Y, \mathscr{Y})$, pointed with their unique 0 -cell, to obtain a presentation $X \otimes Y^{\circ}$ of the prob $(T, \mathscr{T}) \otimes(S, \mathscr{S})$

Even if $(X, \mathscr{X})$ and $(Y, \mathscr{Y})$ contain no generating cells in dimension higher than $3, X \oplus Y^{\circ}$ contains generating cells up to dimension 6 , two more than the threshold of significance for $\mathrm{G}$. Thus the smash product $X \otimes Y^{\circ}$ contains strictly more data than the tensor product $(T, \mathscr{T}) \otimes(S, \mathscr{S})$.

We suggest that these data can be interpreted through the lens of higher-dimensional rewriting, and in particular the concepts of syzygies and coherence; we refer to Yves Guiraud's thèse d'habilitation [Gui19] for an introduction.

Rewriting theory is concerned with computational properties of presentations, in particular the properties of confluence and termination. When a presentation is embodied by a polygraph, confluence at a critical branching is exhibited by a pair of parallel cells. In a coherent presentation, this is strengthened to the requirement that the parallel pair be filled by a higher-dimensional cell, sometimes called a syzygy [Lod00]. This requirement can be extended by asking that higher-dimensional parallel pairs also have fillers.

As the following example shows, it appears that the higher-dimensional cells produced by the smash product of two presentations of pros are syzygies for the presentation of their tensor product.

Example 6.8. Let $(X, \mathscr{X})$ be the presentation of Mon from Example 6.6. Then $\left(X^{\circ}, \mathscr{X}^{\circ}\right)$ is a presentation of $M o n^{\mathrm{co}}$, so the smash product $X \otimes X$ is a presentation of the prob Bialg of bialgebras.

Let us compute this smash product. To simplify, we employ the following abuse of notation: we represent a 3 -diagram $x$ in $X \otimes X$ as a 2-diagram in Bialg whose image through $\mathrm{U}_{3}$ has the same composite as $\mathrm{G} x$. This allows us to depict $n$-cells in $X \otimes X$ as if they were $(n-1)$-cells. This is not a faithful representation: most notably, a subdiagram of a 3-diagram is different from a subdiagram of its representation as a 2-diagram. A 4-diagram may look here like a "3-diagram modulo the axioms of braidings". 
To begin, $X \oplus X$ has a single generating 0-cell and no generating 1-cells. The only generating 2 -cell is $1 \otimes 1$.

The generating 3 -cells are $\mu \otimes 1, \eta \otimes 1,1 \otimes \mu$, and $1 \otimes \eta$. These are the standard generators of Bialg as in Example 5.9, and with our abuse of notation, we use the same depiction:
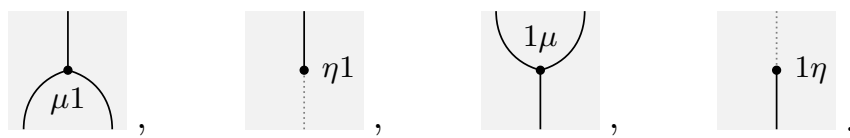

There are 10 generating 4-cells which can be subdivided into three groups. Those of the form $x \otimes 1$ for $x \in \mathscr{X}_{3}$ have the same representation as $x$, that is,
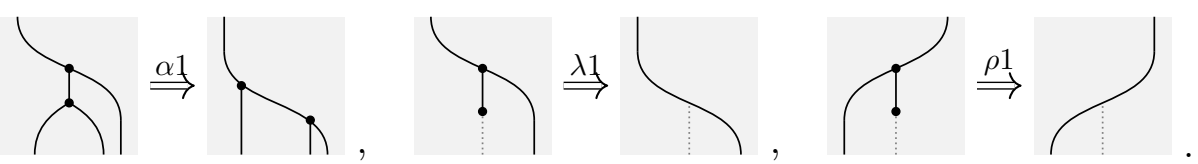

Those of the form $1 \otimes x$ for $x \in \mathscr{X}_{3}$ have the same representation as $x^{\circ}$ :
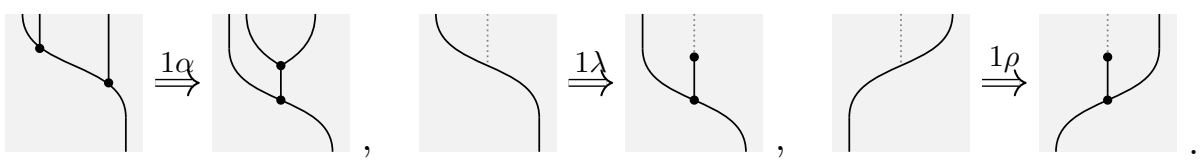

Finally, those of the form $x \otimes y$ for $x, y \in \mathscr{X}_{2}$ present the additional equations of Example 5.9 with the orientation

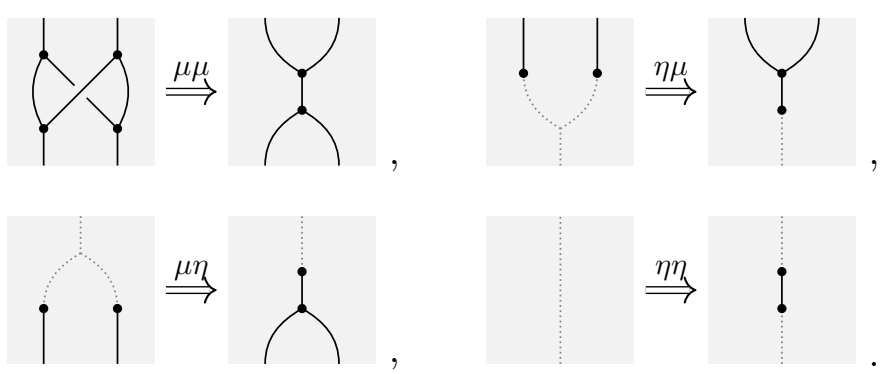

This presentation of Bialg contains new critical branchings that do not correspond to critical branchings in the presentations of Mon or $\mathrm{Mon}^{\mathrm{co}}$. For example, we have the following critical branching involving $\alpha \otimes 1$ and $\mu \otimes \mu$ :

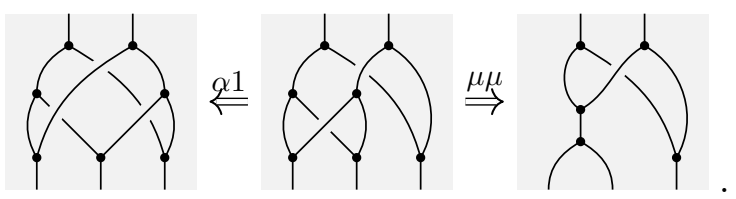

There are 12 generating 5-cells of $X \otimes X$, of the form $x \otimes y$ where either $x \in \mathscr{X}_{3}$ and $y \in \mathscr{X}_{2}$ or $x \in \mathscr{X}_{2}$ and $y \in \mathscr{X}_{3}$. We observe that these are syzygies exhibiting confluence at these critical branchings. 
For example, $\partial^{-}(\alpha \otimes \mu)$ is

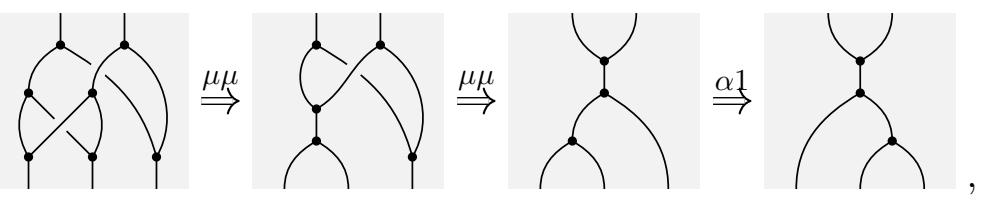

while $\partial^{+}(\alpha \otimes \mu)$ is

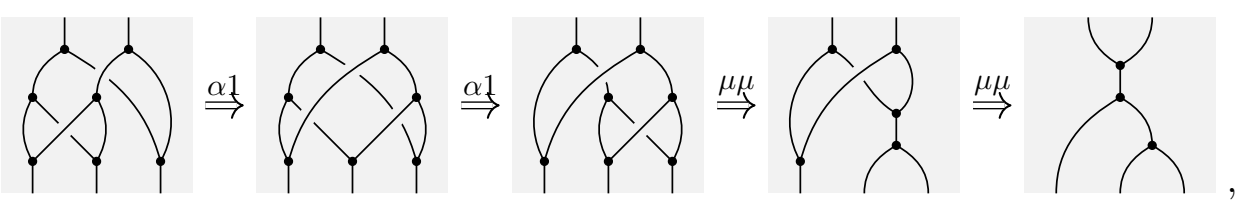

which exhibits confluence at the critical branching (21).

As another example, $\partial^{-}(\eta \otimes \lambda)$ is

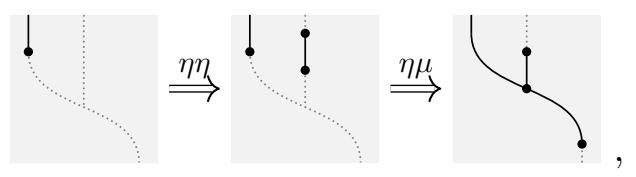

while $\partial^{+}(\eta \otimes \lambda)$ is

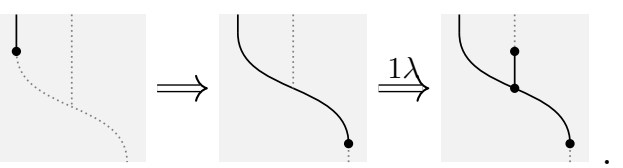

Here the unlabelled 4-cell is a degenerate 4-cell $[\operatorname{Had} 20 \mathrm{~b}, \S 4.14]$ of the form $\eta \otimes x$ where $x$ is a unitor 2-cell. It is mapped by $\mathrm{G}$ to a diagram in Bialg whose image is the unit $\varepsilon(\eta \otimes 1)$, so we may want to treat $\partial^{+}(\eta \otimes \lambda)$ as a single rewrite step. Thus $\eta \otimes \lambda$ exhibits confluence at a critical branching involving $\eta \otimes \eta$ and $1 \otimes \lambda$.

These syzygies are oriented, so they can be interpreted as higher-dimensional rewrites creating critical branchings one dimension up. The 9 generating 6-cells of $X \otimes X$, of the form $x \otimes y$ where $x \in \mathscr{X}_{3}$ and $y \in \mathscr{X}_{3}$, are higher syzygies exhibiting confluence at these higher branchings.

Comment 6.9. Diagrammatic complexes are closely related to polygraphs, so the definitions of confluent, terminating, and coherent presentations should admit sufficiently straightforward translations to our framework.

The only difficulty is the treatment of degenerate cells. This can most likely be circumvented by considering finite sub-presheaves of the underlying combinatorial polygraph of a diagrammatic complex [Had20b, Section 6.2]. We 
note that, in fact, a combinatorial polygraph is equivalent to a polygraph if [Conjecture 7.7, ibid.] holds, which for now is proven up to dimension 3.

We would like to make the informal conjecture that, if $(X, \mathscr{X})$ and $(Y, \mathscr{Y})$ are two presentations of pros that are coherent in a suitable sense, then $X \otimes Y^{\circ}$ is a coherent diagrammatic presentation of their tensor product; or, at least, a coherent presentation can be extracted from it. We leave the formal development of this problem to future work.

6.10. To conclude, we may want to leave behind the interpretation of diagrammatic complexes as presentations of pros or probs and consider them directly as embodiments of higher-dimensional theories, such as homotopical algebraic theories.

6.11 ( $n$-Tuply monoidal diagrammatic set). For each $n>0$, a pointed diagrammatic set $(X, \bullet)$ is $n$-tuply monoidal if $\bullet: 1 \rightarrow \sigma_{\leq n-1} X$ is an isomorphism.

We say monoidal instead of 1-tuply monoidal and doubly monoidal instead of 2-tuply monoidal.

Example 6.12. A presentation of a pro is monoidal, while a presentation of a prob is doubly monoidal.

6.13. We propose the following basic setup for higher-dimensional universal algebra in diagrammatic sets:

- a presentation of a $k$-tuply monoidal higher-dimensional theory is embodied by a $k$-tuply monoidal diagrammatic complex $\left(X, \mathscr{X}, \bullet_{X}\right)$;

- a "semantic universe" for such a theory is a $k$-tuply monoidal diagrammatic set with weak composites $\left(M, \bullet_{M}\right)[\operatorname{Had} 20 \mathrm{~b}, \S 6.1]$, a form of weak higher category;

- both the right and the left hom $\left(X, \bullet_{X}\right) \multimap\left(M, \bullet_{M}\right),\left(M, \bullet_{M}\right) \circ\left(X, \bullet_{X}\right)$ are spaces of models of the theory in $M$. These coincide on 0 -cells, which are pointed morphisms from $\left(X, \bullet_{X}\right)$ to $\left(M, \bullet_{M}\right)$, but have different ("lax" or "oplax") higher transformations.

Diagrammatic sets with weak composites encompass strict $\omega$-categories via the diagrammatic nerve construction of [Section 7.2, ibid.] but also homotopy types via the right adjoint of geometric realisation, leading to a strictly more general class of semantic universes compared to the theory of polygraphs.

Example 6.14. We extend the presentation of Example 6.6 to a presentation of the 2-dimensional theory of pseudomonoids [SD97]. 
First we add generating 4-cells $\pi, \tau$ where

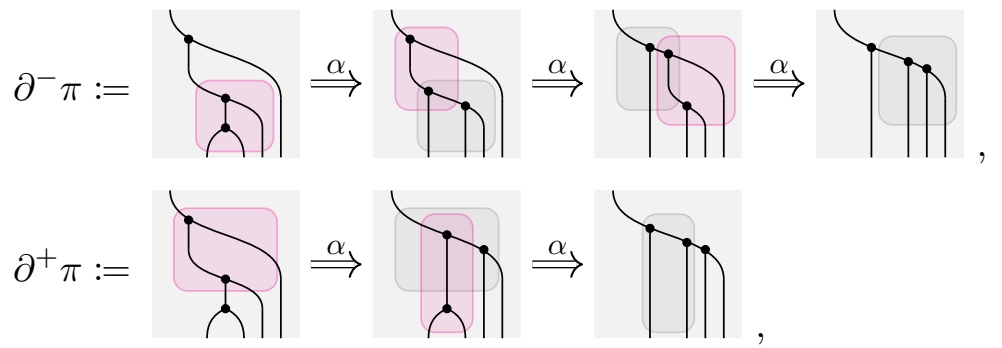

while

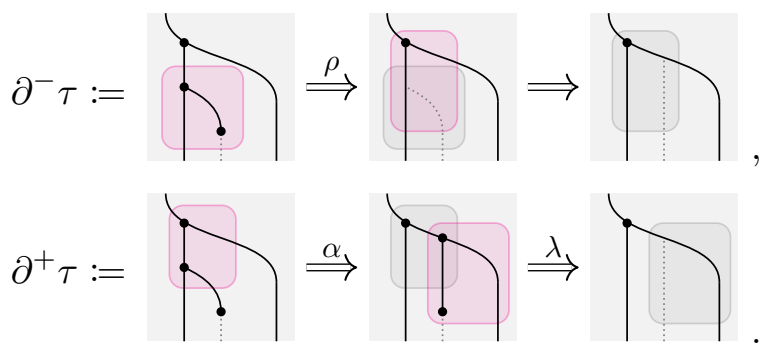

Here the unlabelled 3-cell in $\partial^{-} \tau$ is a degenerate 3 -cell of the form $p ; \mu$ for an appropriate surjective map of atoms $p$.

We let PsMon be the localisation of this diagrammatic complex at the set $\{\alpha, \lambda, \rho\}$ [Had20b, §6.4]. This operation weakly inverts the generating 3-cells. We note that a localisation of a diagrammatic complex is always a diagrammatic complex.

Example 6.15. The paradigmatic 2-category has small categories as 0-cells, functors as 1-cells, and natural transformations as 2-cells. This can be given a cartesian monoidal structure, and this monoidal structure can be strictified, producing a strict monoidal 2-category.

This is equivalent to a 3-category with a single 0-cell. If we restrict to suitably small categories, we can make sure that this defines an object of 3Cat, which through the diagrammatic nerve produces a pointed diagrammatic set $\mathrm{Cat}_{\times}$that has weak composites and is monoidal.

A model of PsMon in $\mathrm{Cat}_{\times}$, that is, a pointed morphism PsMon $\rightarrow \mathrm{Cat}_{\times}$in $\odot$ Set., is then precisely a small monoidal category.

Remark 6.16. Let $\left(X, \bullet_{X}\right)$ be an $n$-tuply monoidal and $\left(Y, \bullet_{Y}\right)$ an $m$-tuply monoidal diagrammatic set. Then $X \otimes Y$ is $(n+m)$-tuply monoidal. As a special case, we recover the fact that the smash product of two presentations of pros is doubly monoidal, so it presents a prob.

Following Baez and Dolan's stabilisation hypothesis [BD95], we expect a $k$-tuply monoidal diagrammatic complex to present a prop when $k>2$. We 
have not defined a realisation functor that would make this statement precise. Nevertheless, this gives us an idea of what iterated smash products of monoidal theories produce: a single smash product yields a braided monoidal theory, any number above that a symmetric monoidal theory.

For higher-dimensional theories, this ought to generalise as follows: the smash product of $m$ different $k$-tuply monoidal diagrammatic sets, interpreted as presentations of $n$-dimensional theories, presents a symmetric monoidal $n$-dimensional theory as soon as $m k>n+1$.

\section{Acknowledgements}

This work was supported by the ESF funded Estonian IT Academy research measure (project 2014-2020.4.05.19-0001).

I am grateful to Simon Forest, Fosco Loregian, François Métayer, Samuel Mimram, Paweł Sobociński, and Sophie Turner for various forms of help.

\section{REFERENCES}

[AM20] D. Ara and G. Maltsiniotis. Joint et tranches pour les $\infty$-catégories strictes. Mémoires de la Société Mathématique de France, 165, 2020.

[AR94] J. Adámek and J. Rosický. Locally presentable and accessible categories, volume 189. Cambridge University Press, 1994.

[Bae06] J.C. Baez. Universal algebra and diagrammatic reasoning. Lectures at Geometry of Computation meeting. Transparencies available at https://math.ucr.edu/home/baez/universal/, 2006.

[BD95] J.C. Baez and J. Dolan. Higher-dimensional algebra and topological quantum field theory. Journal of Mathematical Physics, 36(11):6073-6105, 1995.

[BSZ14] F. Bonchi, P. Sobociński, and F. Zanasi. Interacting bialgebras are Frobenius. In International Conference on Foundations of Software Science and Computation Structures, pages 351-365. Springer, 2014.

[BSZ17] F. Bonchi, P. Sobociński, and F. Zanasi. Interacting Hopf algebras. Journal of Pure and Applied Algebra, 221(1):144-184, 2017.

[Bur93] A. Burroni. Higher-dimensional word problems with applications to equational logic. Theoretical Computer Science, 115(1):43-62, 1993.

[BV06] J.M. Boardman and R.M. Vogt. Homotopy invariant algebraic structures on topological spaces, volume 347. Springer, 2006.

[Car18] J.S. Carter. Graphs, foams, tensors, polytopes, and homology. Journal of Knot Theory and Its Ramifications, 27(11):1843015, 2018. 
[CG07] E. Cheng and N. Gurski. The periodic table of $n$-categories for low dimensions I: degenerate categories and degenerate bicategories. Contemporary Mathematics, 431:143-164, 2007.

[CG11] E. Cheng and N. Gurski. The periodic table of $n$-categories II: degenerate tricategories. Cahiers de topologie et géométrie différentielle catégoriques, $52(2): 82-125,2011$.

[Day70] B. Day. On closed categories of functors. In Lecture Notes in Mathematics, pages 1-38. Springer Berlin Heidelberg, 1970.

[Dor18] C. Dorn. Associative n-categories. PhD thesis, University of Oxford, 2018.

[FM18] S. Forest and S. Mimram. Coherence of Gray categories via rewriting. In 3rd International Conference on Formal Structures for Computation and Deduction (FSCD 2018), volume 108, 2018.

[Fox76] T. Fox. Coalgebras and cartesian categories. Communications in Algebra, 4(7):665-667, 1976.

[GM16] Y. Guiraud and P. Malbos. Polygraphs of finite derivation type. Mathematical Structures in Computer Science, pages 1-47, 2016.

[GPS95] R. Gordon, J. Power, and R. Street. Coherence for tricategories, volume 558. American Mathematical Soc., 1995.

[Gra74] J.W. Gray. Formal category theory: adjointness for 2-categories, volume 391. Springer, 1974.

[Gui19] Y. Guiraud. Rewriting methods in higher algebra. Thèse d'habilitation à diriger des recherches, Université Paris 7, 2019.

[Had17] A. Hadzihasanovic. The algebra of entanglement and the geometry of composition. PhD thesis, University of Oxford, 2017.

[Had20a] A. Hadzihasanovic. A combinatorial-topological shape category for polygraphs. Applied Categorical Structures, 28(3):419-476, 2020.

[Had20b] A. Hadzihasanovic. Diagrammatic sets and rewriting in weak higher categories. arXiv preprint arXiv:2007.14505, 2020.

[HR15] P. Hackney and M. Robertson. On the category of props. Applied Categorical Structures, 23(4):543-573, 2015.

[JS91] A. Joyal and R. Street. The geometry of tensor calculus, I. Advances in mathematics, 88(1):55-112, 1991.

[KV94] M.M. Kapranov and V.A. Voevodsky. 2-categories and Zamolodchikov tetrahedra equations. In Proc. Symp. Pure Math, volume 56, pages 177-260, 1994.

[Lac04] S. Lack. Composing PROPs. Theory and Applications of Categories, 13(9):147-163, 2004. 
[Lac11] S. Lack. A Quillen model structure for Gray-categories. Journal of KTheory, 8(2):183-221, 2011.

[Law63] F.W. Lawvere. Functorial semantics of algebraic theories. Proceedings of the National Academy of Sciences of the United States of America, 50(5):869, 1963.

[Lin69] F.E.J. Linton. Coequalizers in categories of algebras. In Seminar on triples and categorical homology theory, pages 75-90. Springer, 1969.

[Lod00] J.-L. Loday. Homotopical syzygies. Contemporary Mathematics, 265:99$128,2000$.

[Mak05] M. Makkai. The word problem for computads. Available on the author's web page http://www.math.mcgill.ca/makkai, 2005.

[Mim14] S. Mimram. Towards 3-dimensional rewriting theory. Logical Methods in Computer Science, 10(1):1-47, 2014.

[ML65] S. Mac Lane. Categorical algebra. Bulletin of the American Mathematical Society, 71(1):40-106, 1965.

[ML71] S. Mac Lane. Categories for the Working Mathematician. Springer New York, 1971.

[Pir02] T. Pirashvili. On the PROP corresponding to bialgebras. Cahiers de topologie et géométrie différentielle catégoriques, 43(3):221-239, 2002.

[Pow91] J. Power. An n-categorical pasting theorem. In Lecture Notes in Mathematics, pages 326-358. Springer Nature, 1991.

[Rie17] E. Riehl. Category theory in context. Courier Dover Publications, 2017.

[RV19] D. Reutter and J. Vicary. High-level methods for homotopy construction in associative $n$-categories. In 2019 34th Annual ACM/IEEE Symposium on Logic in Computer Science (LICS), pages 1-13. IEEE, 2019.

[SD97] R. Street and B. Day. Monoidal bicategories and Hopf algebroids. Adv. Math, 129:99-157, 1997.

[Sel10] P. Selinger. A survey of graphical languages for monoidal categories. In New Structures for Physics, pages 289-355. Springer, 2010.

[Sim09] C. Simpson. Homotopy Theory of Higher Categories. Cambridge University Press, 2009.

[Ste93] R. Steiner. The algebra of directed complexes. Applied Categorical Structures, 1(3):247-284, 1993.

[Ste04] R. Steiner. Omega-categories and chain complexes. Homology, Homotopy and Applications, 6(1):175-200, 2004.

[VD19] J. Vicary and L. Dunn. Coherence for Frobenius pseudomonoids and the geometry of linear proofs. Logical Methods in Computer Science, 15(3), 2019. 